\title{
Morphological and phylogenetic characterization of novel pestalotioid species associated with mangroves in Thailand
}

\author{
Norphanphoun $\mathbf{C}^{1,2,3}$, Jayawardena $\mathbf{R S}^{2}$, Chen $\mathbf{Y}^{4}$, Wen $\mathbf{T C}^{1,3}$, Meepol $\mathbf{W}^{5}$, \\ Hyde KD ${ }^{2, *}$
}

\begin{abstract}
${ }^{1}$ The Engineering Research Center of Southwest Bio-Pharmaceutical Resources, Ministry of Education, Guizhou University, Guiyang, 550025, China

${ }^{2}$ Center of Excellence in Fungal Research, Mae Fah Luang University, Chiang Rai, 57100, Thailand

${ }^{3}$ State Key Laboratory Breeding Base of Green Pesticide and Agricultural Bioengineering, Key Laboratory of Green Pesticide and Agricultural Bioengineering, Ministry of Education, Guizhou University, Guiyang 550025, China

${ }^{4}$ Law Enforcement of Agricultural Bureau, Xiu Wen district, Guiyang City, Guizhou Province 550200, China

${ }^{5}$ Ranong Mangrove Forest Research Center, Department of Marine and Coastal Resources, Tambon Ngao, Muang District, Ranong 85000, Thailand
\end{abstract}

Norphanphoun C, Jayawardena RS, Chen Y, Wen TC, Meepol W, Hyde KD 2019 - Morphological and phylogenetic characterization of novel pestalotioid species associated with mangroves in Thailand. Mycosphere 10(1), 531-578, Doi 10.5943/mycosphere/10/1/9

\begin{abstract}
Pestalotioid fungi are associated with a wide variety of plants worldwide and are endophytes, pathogens and saprobes. The present study provides an updated phylogenetic placement of Neopestalotiopsis, Pestalotiopsis and Pseudopestalotiopsis using fresh collections from mangrove plants in Thailand. Twelve novel species are characterized based on combined sequence data analyses of internal transcribed spacer (ITS), beta tubulin ( $\beta$-tubulin) and translation elongation factor 1-alpha $(E F 1 \alpha)$ coupled with morphological characters. The taxonomy and phylogenetic relationships of pestalotioid-fungi are reappraised with suggestions for future work.
\end{abstract}

Keywords - 12 new species - Asymptomatic leaves - Acrostichum aureum - Avicenia marina Leaf spots - Mangroves - Neopestalotiopsis - Pestalotiopsis - pestalotiopsis-like - Phylogeny Pseudopestalotiopsis - Rhizophora sp. - Sonneronata alba - Taxonomy

\section{Introduction}

Mangrove forests are one of the most productive ecosystems which play a major role in the ecological communities in coastal tropical and subtropical waters (Hyde \& Lee 1995). They serve as hatcheries and nursery habitats for marine organisms and protect coastlines from catastrophic events such as storms and tidal surges (Hyde \& Jones 1988, Fisher \& Spalding 1993, Hyde \& Lee 1995, Hyde et al. 1998). The greatest diversity of mangrove species occurs in Indonesia, Malaysia and Thailand, which also harbour a great diversity of micro-organisms especially fungi (Alias \& Jones 2009, Alias et al. 2010). However, the phylogenetic and functional description of microbial diversity in mangrove ecosystems has not been addressed to the same extent as for other environments. Even though mangrove ecosystems are very rich in microbial diversity, less than 5\% of species are believed to have been described (Thatoi et al. 2012). Recently developed technologies in molecular biology and genetics offer a great promise to explore the potential of microbial diversity. Hence, the present paper makes an effort to identify the species of fungi in 
mangrove ecosystems in Thailand (Guba 1961, Barr 1975, Nag Raj 1993, Norphanphoun et al. 2018, Kumar et al. 2019).

Several studies have focused on the diversity and the ecology of fungi in this special habitat and there are relatively few studies on disease-causing fungi of mangrove (Cribb \& Cribb 1955, Kohlmeyer \& Kohlmeyer 1979, Hyde \& Jones 1988). Most of the mangrove fungi are reported as saprobes and endophytes (e.g Hu et al. 2007, Jones et al. 2009, Pang et al. 2010, Doilom et al. 2017, Devadatha et al. 2018, Li et al. 2018, Kumar et al. 2019). Zhou et al. (2018) reported that the endophytic fungi Diaporthe sp., Neopestalotiopsis protearum and Pestalotiopsis sp. are predominant in mangrove trees in south China. Endophytic fungal genera such as Cladosporium, Colletotrichum, Fusarium, Pestalotiopsis, Phomopsis, Phyllosticta and Xylaria were also reported from Thailand mangroves (Chaeprasert et al. 2010, Doilom et al. 2017). Nevertheless, endophytic fungi are less well-studied and studies mainly identified taxa to the genus level (Chaeprasert et al. 2010, Abraham et al. 2015, Thomas et al. 2016). Pathogenic mangrove fungi have received much less attention (e.g Hyde \& Cannon 1992, Jones \& Pang 2012, Jones et al. 2019). In the present study we focused on Pestalotioid taxa on Acrostichum aureum, Avicennia marina, Rhizophora (mainly $R$. apiculata and $R$. mucronata) and Sonneronata alba, which were abundant plants in Thailand mangroves. The most notable mangrove plant among Thailand mangroves is Rhizophora, a genus of tropical mangrove trees, which are collectively known as true mangroves (Hassan et al. 2018).

Pestalotioid fungi are characterized based on morphological features such as the median cell colour (Maharachchikumbura et al. 2011). They are common phytopathogens causing a variety of diseases or saprobes or endophytes, and are widely distributed in tropical and temperate regions (Guba 1961, Barr 1975, Nag Raj 1993, Maharachchikumbura et al. 2014). However, species identification in this genus remains a major challenge because of overlapping morphologies (Jeewon et al 2002, 2003, Maharachchikumbura et al. 2012, 2011). Maharachchikumbura et al. (2014) split Pestalotiopsis into three genera: Neopestalotiopsis, Pseudopestalotiopsis and Pestalotiopsis, based on conidia pigment colour, conidiophores and molecular phylogeny. Neopestalotiopsis can be easily distinguished from Pseudopestalotiopsis and Pestalotiopsis by its versicolourous median cells (Maharachchikumbura et al. 2014). Pseudopestalotiopsis differs from Pestalotiopsis by having darker three median cells and knobbed apical appendages (Maharachchikumbura et al. 2014). Many novel species were introduced into this group during recent years (Maharachchikumbura et al. 2016, Liu et al. 2017, Nozawa et al. 2017, Ariyawansa et al. 2018, Watanabe et al. 2018, Tsai et al. 2018, Tibpromma et al. 2018). This study aimed to identify the pestalotioid fungi associated with mangroves in Thailand based on both morphological characters and molecular phylogeny.

\section{Materials \& Methods}

\section{Sample collection and examination of specimens}

Fresh leaf samples (both diseased and healthy) were collected in 2016, from Acrostichum aureum, Avicenia marina, Rhizophora apiculata, R. mucronata and Sonneronata alba from Phetchaburi, Ranong and Trat provinces, Thailand. Permission for collecting specimens were obtained where necessary. Fresh specimens were taken to the laboratory in paper bags, examined and described following Norphanphoun et al. (2017). Morphological characters of conidiomata were examined using a Motic SMZ 168 dissecting microscope (Motic Incorporation Ltd., Hong Kong). Hand sections were mounted in water and examined for morphological details. Micromorphology was studied using a Nikon Ni compound microscope (Nikon Instruments Inc., NY, USA) fitted with a Canon EOS 600D digital camera (Canon Inc., Tokyo, Japan). Photo-plates were made using Adobe Photoshop CS6 Extended version 13.0 × 64 (Adobe Systems, CA, USA), while Tarosoft (R) Image Frame Work program v. 0.9.7 (Tarosoft, Thailand) was used for measurements.

Cultures were obtained by the tissue isolation method outlined in Norphanphoun et al. (2018). Single hyphal tips were transferred onto potato dextrose agar (PDA, Hardy Diagnostics, CA, USA) 
plates throughout a two-week period. Pure cultures were maintained for further studies on PDA. The specimens/dried cultures and living cultures are deposited in the Herbarium Mae Fah Luang University (MFLU) and culture collection Mae Fah Luang University (MFLUCC), Chiang Rai, Thailand and duplicated in the International Collection of Micro-organisms from Plants (ICMP). Facesoffungi numbers were obtained as in Jayasiri et al. (2015) and the new species were registered in Index Fungorum (Index Fungorum 2019).

\section{DNA extraction, PCR amplification and sequencing}

Genomic DNA was extracted from fresh fungal mycelia growing on PDA at room temperature $\left(18-25^{\circ} \mathrm{C}\right)$ for two weeks using a E.Z.N.A. ${ }^{\text {TM }}$ Fungal DNA MiniKit (Omega Biotech, CA, USA) following the manufacturer's protocols. Polymerase chain reactions (PCR) were carried out using primer pairs of ITS1/ITS4 to amplify the ITS region (White et al. 1990), EF1-728f/EF2 to amplify EF1 $\alpha$ (Carbone \& Kohn 1999, O'Donnell et al. 1998) and the partial $\beta$-tubulin region was amplified using primers T1/Bt2b (Glass \& Donaldson 1995, O'Donnell \& Cigelnik 1997).

The amplification reactions were carried out with the following protocol: $50 \mu 1$ reaction volume containing $2 \mu \mathrm{l}$ of DNA template, $2 \mu \mathrm{l}$ of each forward and reverse primers, $25 \mu \mathrm{l}$ of $2 \times$ Bench Top ${ }^{\mathrm{TM}}$ Taq Master Mix (mixture of Taq DNA Polymerase (recombinant): 0.05 units $/ \mu \mathrm{L}$, $\mathrm{MgCl}_{2}$ : $4 \mathrm{mM}$, and dNTPs (dATP, dCTP, dGTP, dTTP): $0.4 \mathrm{mM}$ ) and $19 \mu \mathrm{l}$ of double-distilled water $\left(\mathrm{ddH}_{2} \mathrm{O}\right)$ (sterilized water). The PCR thermal cycle program for each gene is described in Table 1. Purification and sequencing of PCR products with the same primers mentioned above were carried out at Life Biotechnology Co., Shanghai, China.

Table 1 Polymerase chain reactions (PCR) thermal cycle program for each gene.

\begin{tabular}{|c|c|c|}
\hline Gene & Primers & PCR thermal cycle protocals \\
\hline ITS & ITS1/ITS4 & $\begin{array}{l}\text { initially } 95{ }^{\circ} \mathrm{C} \text { for } 3 \mathrm{~min} \text {, followed by } 40 \text { cycles of denaturation at } \\
95^{\circ} \mathrm{C} \text { for } 30 \mathrm{~s} \text {, annealing at } 55^{\circ} \mathrm{C} \text { for } 50 \mathrm{~s} \text {, elongation at } 72{ }^{\circ} \mathrm{C} \text { for } 1 \\
\text { min, final extension at } 72{ }^{\circ} \mathrm{C} \text { for } 7 \mathrm{~min}\end{array}$ \\
\hline$\beta$-tubulin & $\mathrm{T} 1 / \mathrm{Bt} 2 \mathrm{~b}$ & $\begin{array}{l}\text { initially } 95{ }^{\circ} \mathrm{C} \text { for } 3 \mathrm{~min} \text {, followed by } 40 \text { cycles of denaturation at } \\
94{ }^{\circ} \mathrm{C} \text { for } 30 \mathrm{~s} \text {, annealing at } 55^{\circ} \mathrm{C} \text { for } 50 \mathrm{~s} \text {, elongation at } 72^{\circ} \mathrm{C} \text { for } 1 \\
\text { min, final extension at } 72{ }^{\circ} \mathrm{C} \text { for } 7 \mathrm{~min}\end{array}$ \\
\hline $\mathrm{EF} 1 \alpha$ & EF1-728f/EF2 & $\begin{array}{l}\text { initially } 94{ }^{\circ} \mathrm{C} \text { for } 5 \mathrm{~min} \text {, followed by } 40 \text { cycles of denaturation at } \\
94{ }^{\circ} \mathrm{C} \text { for } 30 \mathrm{~s} \text {, annealing at } 52{ }^{\circ} \mathrm{C} \text { for } 30 \mathrm{~s} \text {, elongation at } 72{ }^{\circ} \mathrm{C} \text { for } \\
\text { 30s, final extension at } 72{ }^{\circ} \mathrm{C} \text { for } 7 \mathrm{~min}\end{array}$ \\
\hline
\end{tabular}

\section{Phylogenetic analysis}

The sequences were assembled by Geneious® 11.1 .5 (http://www.geneious.com). Multiple alignments derived in this study were analysed with similar sequences, acquired from GenBank BLASTn queries and recently published articles (Maharachchikumbura et al. 2016, Liu et al. 2017, Nozawa et al. 2017, Tsai et al. 2018, Watanabe et al. 2018). ITSx 1.1 (a Perl-based software tool) was used to extract ITS1, 5.8S and ITS2 (Bengtsson-Palme et al. 2013). BioEdit 7.2.3 (Hall 1999) was used to extract the partition of $\beta$-tubulin, and EF1 $\alpha$, which follow the partition template based on nucleotide BLAST in GenBank. Combined analyses of ITS1+ITS2, 5.8S, $\beta$-tubulin and EF1 $\alpha$ sequence data were performed using maximum parsimony (MP), maximum likelihood (ML) and Bayesian analysis (BI) for each genus separately. The dataset of Neopestalotiopsis consisted of 52 taxa (outgroup: Pestalotiopsis diversiseta MFLUCC 12-0287); 70 taxa for Pestalotiopsis (outgroup: Neopestalotiopsis saprophytica MFLUCC 12-0282) and 27 taxa for Pseudopestalotiopsis (outgroup: Neopestalotiopsis natalensis CBS 138.41). All sequences were aligned for each gene separately using MAFFT v.7.110 online program (http://mafft.cbrc.jp/alignment/server/; Katoh \& Standley 2013) and Gblocks v. 0.91b was used to exclude ambiguously aligned positions in the ITS, EF1 $\alpha$ and $\beta$-tubulin alignments (Castresana 2000, Talavera \& Castresana 2007). A partition homogeneity test (PHT) was performed with 
PAUP* 4.0b10 to determine whether the individual datasets were congruent and could be combined (Swofford 2002). The combined sequence alignments were obtained from MEGA7 version 7.0.14 (Kumar et al. 2015) and missing data were coded as gap (-) and further manual adjustments were made wherever necessary in BioEdit 7.2.3 (Hall 1999). The combined sequence alignment was converted to NEXUS file for maximum parsimony analysis using ClustalX v. 2 (Larkin et al. 2007). The NEXUS file was prepared for MrModeltest v. 2.2 (Nylander 2004) in PAUP* v.4.0b10 (Swofford 2002).

Maximum parsimony (MP) analysis was performed using PAUP* v. 4.0b10 (Swofford 2002) with 1000 bootstrap replicates using heuristic search with random stepwise addition and treebisection reconnection (TBR). Maxtrees were set to 1000 and branches of zero length were collapsed. The following descriptive tree statistics were calculated: parsimony tree length (TL), consistency index (CI), retention index (RI), rescaled consistency index (RC) and homoplasy index (HI).

For both maximum likelihood and Bayesian analyses, a partitioned analysis was performed with the following six partitions: ITS1+ITS2, 5.8S, $\beta$-tubulin (exon), $\beta$-tubulin (intron), EF1 $\alpha$ (exon), and EF1 $\alpha$ (intron). Maximum-likelihood (ML) analysis was performed with RAxML (Stamatakis 2006) implemented in the CIPRES Science Gateway web server (RAxML-HPC2on XSEDE; Miller et al. 2010), 1000 rapid bootstrap replicates were run with GTRGAMMA model of nucleotide evolution. Maximum likelihood bootstrap values (MLBS) equal or greater than 50\% are presented above each node in the resulting phylogenetic trees.

Bayesian inference (BI) analysis was performed using the Markov Chain Monte Carlo (MCMC) method with MrBayes 3.2.2 (Ronquist et al. 2012). The best-fit nucleotide substitution model for each dataset was separately determined using MrModeltest version 2.2 (Nylander 2004). GTR model was selected a best-fit model for the $\beta$-tubulin-exons (Neopestalotiopsis), GTR $+\mathrm{I}$ model was selected a best-fit model for the $\beta$-tubulin-exons (Pseudopestalotiopsis), ITS1+ITS2+5.8S (Neopestalotiopsis), GTR $+\mathrm{I}+\mathrm{G}$ model for $\beta$-tubulin-exons (Pestalotiopsis), HKY model for ITS1+ITS2+5.8S (Pseudopestalotiopsis), HKY $+\mathrm{G}$ model for EF1 $\alpha$-introns (Neopestalotiopsis), EF1 $\alpha$-introns (Pestalotiopsis), and $\beta$-tubulin-introns (Pseudopestalotiopsis), $\mathrm{HKY}+\mathrm{I}$ model for EF1 $\alpha$-introns (Pseudopestalotiopsis), HKY $+\mathrm{I}+\mathrm{G}$ model for ITS1+ITS2+5.8S (Pestalotiopsis) and $\beta$-tubulin-introns (Pestalotiopsis), $\mathrm{K} 80$ model for EF1 $\alpha$-exons (Pseudopestalotiopsis), K80+G model for $\beta$-tubulin-introns (Neopestalotiopsis), SYM model for EF1 $\alpha$-exons (Neopestalotiopsis), SYM+I model was selected a best-fit model for the EF1 $\alpha$-exons (Pestalotiopsis). The MCMC analyses, with four chains starting from random tree topology, were run between 1,000,000-5,000,000 generations for each combined dataset. Trees were sampled every 100 generations. Tracer v. 1.5.0 was used to check parameters including the effective sampling sizes (ESS) >200, the stable likelihood plateaus and burn-in value (Rambaut et al. 2013). The first 5000 samples were excluded as burn-in.

The phylogram was visualized in FigTree v1.4.0 (http://tree.bio.ed.ac.uk/software /figtree/; Rambaut 2014) and made in Adobe Illustrator CC (Adobe Systems, CA, U.S.A.) and Adobe Photoshop CS6 Extended version 13.1.2 × 64. Newly generated sequences in this study are deposited in GenBank (Table 2). The finalized alignments and trees were deposited in TreeBASE. The phylogram was visualized in FigTree v1.4.0 (http://tree.bio.ed.ac.uk/software/figtree/; Rambaut 2014) and made in Adobe Illustrator CC (Adobe Systems, CA, U.S.A.) and Adobe Photoshop CS6 Extended version 13.1.2 × 64. Newly generated sequences in this study are deposited in GenBank (Table 2). The finalized alignments and trees were deposited in TreeBASE, Fig. 1: submission ID: 24243 (Reviewer access URL: http://purl.org/phylo/treebase/phylows/ study/TB2:S24243? $\mathrm{x}$-access-code=9556bf0e6bb1598ab7df74b6c8c86564\&format=html), Fig. 8 : submission ID: 24244 (Reviewer access URL: http://purl.org/phylo/treebase/phylows/study/TB2: S24244? $\mathrm{x}$-access-code=83ce9ad2fa86038ae180067b214435b2\&format=html), Fig. 11: submission ID: 24245 (Reviewer access URL: http://purl.org/phylo/treebase/phylows/study/TB2:S24245?xaccess-code $=\mathrm{cd} 7080 \mathrm{~d} 4 \mathrm{e} 86 \mathrm{cc} 3268 \mathrm{e} 7981 \mathrm{fcf} 2 \mathrm{~b} 7 \mathrm{fc} 47 \&$ format $=\mathrm{html})$. 
Table 2 GenBank accession numbers of the sequences used in phylogenetic analyses.

\begin{tabular}{|c|c|c|c|c|c|c|}
\hline Taxa & Strain number $^{\mathrm{a}}$ & Host & Country & ITS & $\beta$-tubulin & EF1a \\
\hline \multirow[t]{2}{*}{ Neopestalotiopsis acrostichi } & MFLUCC 17-1754 ${ }^{\mathrm{T}}$ & Acrostichum aureum & Thailand & MK764272 & MK764338 & MK764316 \\
\hline & MFLUCC 17-1755 & Acrostichum aureum & Thailand & MK764273 & MK764339 & MK764317 \\
\hline N. brachiata & MFLUCC $17-1555^{\mathrm{T}}$ & Rhizophora apiculata & Thailand & MK764274 & MK764340 & MK764318 \\
\hline \multirow[t]{2}{*}{ N. petila } & MFLUCC 17-1738 ${ }^{\mathrm{T}}$ & Rhizophora mucronata & Thailand & MK764275 & MK764341 & MK764319 \\
\hline & MFLUCC 17-1737 & Rhizophora mucronata & Thailand & MK764276 & MK764342 & MK764320 \\
\hline \multirow[t]{2}{*}{ N. rhizophorae } & MFLUCC 17-1550 ${ }^{\mathrm{T}}$ & Rhizophora mucronata & Thailand & MK764277 & MK764343 & MK764321 \\
\hline & MFLUCC 17-1551 & Rhizophora mucronata & Thailand & MK764278 & MK764344 & MK764322 \\
\hline \multirow[t]{2}{*}{ N. sonneratae } & MFLUCC 17-1745 ${ }^{\mathrm{T}}$ & Sonneronata alba & Thailand & MK764279 & MK764345 & MK764323 \\
\hline & MFLUCC 17-1744 & Sonneronata alba & Thailand & MK764280 & MK764346 & MK764324 \\
\hline \multirow[t]{2}{*}{ N. thailandica } & MFLUCC 17-1730 ${ }^{\mathrm{T}}$ & Rhizophora mucronata & Thailand & MK764281 & MK764347 & MK764325 \\
\hline & MFLUCC 17-1731 & Rhizophora mucronata & Thailand & MK764282 & MK764348 & MK764326 \\
\hline \multirow[t]{2}{*}{ N. alpapicalis } & MFLUCC $17-2544^{\mathrm{T}}$ & Rhyzophora mucronata & Thailand & MK357772 & MK463545 & MK463547 \\
\hline & MFLUCC 17-2545 & symptomatic leaves $R$. apiculate & Thailand & MK357773 & MK463546 & MK463548 \\
\hline N. aotearoa & CBS $367.54^{\mathrm{T}}$ & Canvas & New Zealand & KM199369 & KM199454 & KM199526 \\
\hline N. asiatica & MFLUCC $12-0286^{\mathrm{T}}$ & unidentified tree & China & JX398983 & JX399018 & JX399049 \\
\hline N. australis & CBS $114159^{\mathrm{T}}$ & Telopea sp. & Australia & KM199348 & KM199432 & KM199537 \\
\hline N. chrysea & MFLUCC $12-0261^{\mathrm{T}}$ & dead leaves & China & JX398985 & JX399020 & JX399051 \\
\hline N. clavispora & MFLUCC $12-0281^{\mathrm{T}}$ & Magnolia sp. & China & JX398979 & JX399014 & JX399045 \\
\hline N. cocoes & MFLUCC $15-0152^{\mathrm{T}}$ & Cocos nucifera & Thailand & NR_156312 & - & KX789689 \\
\hline N. coffea-arabicae & HGUP4015 ${ }^{\mathrm{T}}$ & Coffea arabica & China & KF412647 & KF412641 & KF412644 \\
\hline N. cubana & CBS $600.96^{\mathrm{T}}$ & leaf litter & Cuba & KM199347 & KM199438 & KM199521 \\
\hline N. ellipsospora & MFLUCC $12-0283^{\mathrm{T}}$ & dead plant material & China & JX398980 & JX399016 & JX399047 \\
\hline N. egyptiaca & CBS $140162^{\mathrm{T}}$ & Mangifera indica & Egypt & KP943747 & KP943746 & KP943748 \\
\hline N. eucalypticola & CBS $264.37^{\mathrm{T}}$ & Eucalyptus globulus & - & KM199376 & KM199431 & KM199551 \\
\hline N. foedans & CGMCC $3.9123^{\mathrm{T}}$ & unidentified mangrove plant & China & JX398987 & JX399022 & JX399053 \\
\hline N. formicarum & CBS $362.72^{\mathrm{T}}$ & dead ant & Ghana & KM199358 & KM199455 & KM199517 \\
\hline N. honoluluana & CBS $114495^{\mathrm{T}}$ & Telopea sp. & USA & KM199364 & KM199457 & KM199548 \\
\hline$N$. iraniensis & CBS $137768^{\mathrm{T}}$ & Fragaria $\times$ ananassa & Iran & KM074048 & KM074057 & KM074051 \\
\hline N. javaensis & CBS $257.31^{\mathrm{T}}$ & Cocos nucifera & Indonesia & KM199357 & KM199457 & KM199548 \\
\hline N. keteleeria & MFLUCC $13-0915^{\mathrm{T}}$ & living leaves of Keteleeria pubescens & China & KJ503820 & KJ503821 & KJ503822 \\
\hline N. magna & MFLUCC $12-0652^{\mathrm{T}}$ & Pteridium sp. & France & KF582795 & KF582793 & KF582791 \\
\hline N. mesopotamica & CBS $336.86^{\mathrm{T}}$ & Pinus brutia & Iraq & KM199362 & KM199441 & KM199555 \\
\hline
\end{tabular}


Table 2 Continued.

\begin{tabular}{|c|c|c|c|c|c|c|}
\hline Taxa & Strain number ${ }^{a}$ & Host & Country & ITS & $\beta$-tubulin & EF1a \\
\hline N. musae & MFLUCC $15-0776^{\mathrm{T}}$ & Musa sp. & Thailand & NR_156311 & KX789686 & KX789685 \\
\hline N. natalensis & CBS $138.41^{\mathrm{T}}$ & Acacia mollissima & South Africa & NR_156288 & KM199466 & KM199552 \\
\hline N. pernambucana & GS-2014 RV01 ${ }^{\mathrm{T}}$ & Vismia guianensis & Brazil & KJ792466 & - & - \\
\hline \multirow[t]{3}{*}{ N. piceana } & CBS $394.48^{\mathrm{T}}$ & Picea sp. & UK & KM199368 & KM199453 & KM199527 \\
\hline & CBS 254.32 & Cocos nucifera & Indonesia & KM199372 & KM199452 & KM199529 \\
\hline & CBS 225.3 & Mangifera indica & - & KM199371 & KM199451 & KM199535 \\
\hline \multirow[t]{2}{*}{ N. protearum } & CBS $114178^{\mathrm{T}}$ & Leucospermum cuneiforme cv. "Sunbird" & Zimbabwe & JN712498 & KM199463 & KM199542 \\
\hline & CMM1357 & - & - & KY549597 & KY549632 & KY549594 \\
\hline N. rosae & CBS $101057^{\mathrm{T}}$ & Rosa sp. & New Zealand & KM199359 & KM199429 & KM199523 \\
\hline \multirow[t]{2}{*}{ N. rosicola } & CFCC $51992^{\mathrm{T}}$ & Rosa chinensis & China & KY885239 & KY885245 & KY885243 \\
\hline & CFCC 51993 & Rosa chinensis & China & KY885240 & KY885246 & KY885244 \\
\hline N. samarangensis & MFLUCC $12-0233^{\mathrm{T}}$ & Syzygium samarangense & Thailand & JQ968609 & JQ968610 & JQ968611 \\
\hline N. saprophytica & MFLUCC $12-0282^{\mathrm{T}}$ & Magnolia sp. & China & KM199345 & KM199433 & KM199538 \\
\hline N. steyaertii & IMI $192475^{\mathrm{T}}$ & Eucalyptus viminalis & Australia & KF582796 & KF582794 & KF582792 \\
\hline \multirow[t]{2}{*}{ N. surinamensis } & CBS $450.74^{\mathrm{T}}$ & soil under Elaeis guineensis & Suriname & KM199351 & KM199465 & KM199518 \\
\hline & CBS 111494 & Protea eximia & Zimbabwe & - & KM199462 & KM199530 \\
\hline N. umbrinospora & MFLUCC $12-0285^{\mathrm{T}}$ & unidentified plant & China & JX398984 & JX399019 & JX399050 \\
\hline N. vitis & MFLUCC $15-1265^{\mathrm{T}}$ & Vitis vinifera $\mathrm{cv}$. "Summer black" & China & KU140694 & KU140685 & KU140676 \\
\hline N. zimbabwana & CBS $111495^{\mathrm{T}}$ & Leucospermum cunciforme cv. "Sunbird" & Zimbabwe & JX556231 & KM199456 & KM199545 \\
\hline \multirow[t]{2}{*}{ P. rhizophorae } & MFLUCC 17-0416 ${ }^{\mathrm{T}}$ & Rhizophora apiculata & Thailand & MK764283 & MK764349 & MK764327 \\
\hline & MFLUCC 17-0417 & Rhizophora apiculata & Thailand & MK764284 & MK764350 & MK764328 \\
\hline \multirow[t]{2}{*}{ P. thailandica } & MFLUCC 17-1616 ${ }^{\mathrm{T}}$ & Rhizophora apiculata & Thailand & MK764285 & MK764351 & MK764329 \\
\hline & MFLUCC 17-1617 & Rhizophora apiculata & Thailand & MK764286 & MK764352 & MK764330 \\
\hline P. adusta & ICMP $6088^{\mathrm{T}}$ & on refrigerator door PVC gasket & Fiji & JX399006 & JX399037 & JX399070 \\
\hline P. aggestorum & LC6301 ${ }^{\mathrm{T}}$ & Camellia sinensis & China & KX895015 & KX895348 & KX895234 \\
\hline P. anacardiacearum & IFRDCC $2397^{\mathrm{T}}$ & Mangifera indica & China & KC247154 & KC247155 & KC247156 \\
\hline P. arceuthobii & CBS $434.65^{\mathrm{T}}$ & Arceuthobium campylopodum & USA & KM199341 & KM199427 & KM199516 \\
\hline$P$. arengae & CBS $331.92^{\mathrm{T}}$ & Arenga undulatifolia & Singapore & KM199340 & KM199426 & KM199515 \\
\hline P. australasiae & CBS $114126^{\mathrm{T}}$ & Knightia sp. & New Zealand & KM199297 & KM199409 & KM199499 \\
\hline P. australis & CBS $114193^{\mathrm{T}}$ & Grevillea sp. & Australia & KM199332 & KM199383 & KM199475 \\
\hline
\end{tabular}


Table 2 Continued.

\begin{tabular}{|c|c|c|c|c|c|c|}
\hline Taxa & Strain number ${ }^{a}$ & Host & Country & ITS & $\beta$-tubulin & EF1a \\
\hline P. biciliata & CBS $124463^{T}$ & Platanus $\times$ hispanica & Slovakia & KM199308 & KM199399 & KM199505 \\
\hline P. brachiata & $\mathrm{LC} 2988^{\mathrm{T}}$ & Camellia sp. & China & KX894933 & KX895265 & KX895150 \\
\hline P. brassicae & CBS $170.26^{\mathrm{T}}$ & Brassica napus & New Zealand & KM199379 & - & KM199558 \\
\hline P. camelliae & MFLUCC 12-0277 T & Camellia japonica & China & JX399010 & JX399041 & JX399074 \\
\hline P. chamaeropis & CBS $186.71^{\mathrm{T}}$ & Chamaerops humilis & Italy & KM199326 & KM199391 & KM199473 \\
\hline P. clavata & MFLUCC $12-0268^{\mathrm{T}}$ & Buxus sp. & China & JX398990 & JX399025 & JX399056 \\
\hline P. colombiensis & CBS $118553^{\mathrm{T}}$ & Eucalyptus eurograndis & Colombia & KM199307 & KM199421 & KM199488 \\
\hline P. digitalis & ICMP $5434^{\mathrm{T}}$ & Digitalis purpurea & New Zealand & KP781879 & KP781883 & - \\
\hline P. diploclisiae & CBS $115587^{\mathrm{T}}$ & Diploclisia glaucescens & Hong Kong & KM199320 & KM199419 & KM199486 \\
\hline P. distincta & $\mathrm{LC} 3232^{\mathrm{T}}$ & Camellia sinensis & China & KX894961 & KX895293 & KX895178 \\
\hline P. diversiseta & MFLUCC $12-0287^{\mathrm{T}}$ & dead plant material & China & NR_120187 & JX399040 & JX399073 \\
\hline P. dracontomelon & MFUCC $10-0149^{\mathrm{T}}$ & Dracontomelon dao & Thailand & KP781877 & - & KP781880 \\
\hline P. ericacearum & IFRDCC $2439^{\mathrm{T}}$ & Rhododendron delavayi & China & KC537807 & KC537821 & KC537814 \\
\hline \multirow[t]{2}{*}{ P. formosana } & NTUCC $17-009^{\mathrm{T}}$ & on dead grass & Taiwan & MH809381 & MH809385 & MH809389 \\
\hline & NTUCC 17-010 & on dead grass & Taiwan & MH809382 & MH809386 & MH809390 \\
\hline P. furcata & MFLUCC $12-0054^{\mathrm{T}}$ & Camellia sinensis & Thailand & JQ683724 & JQ683708 & JQ683740 \\
\hline P. gaultheria & IFRD $411-014^{\mathrm{T}}$ & Gaultheria forrestii & China & KC537805 & KC537819 & KC537812 \\
\hline P. gibbosa & NOF $3175^{\mathrm{T}}$ & Gaultheria shallon & Canada & LC311589 & LC311590 & LC311591 \\
\hline P. grevilleae & CBS $114127^{\mathrm{T}}$ & Grevillea sp. & Australia & KM199300 & KM199407 & KM199504 \\
\hline P. hawaiiensis & CBS $114491^{\mathrm{T}}$ & Leucospermum sp. (coral) & USA & KM199339 & KM199428 & KM199514 \\
\hline P. hollandica & CBS $265.33^{\mathrm{T}}$ & Sciadopitys verticillata & The Netherlands & KM199328 & KM199388 & KM199481 \\
\hline P. humus & CBS $336.97^{\mathrm{T}}$ & soil & Papua New Guinea & KM199317 & KM199420 & KM199484 \\
\hline P. inflexa & MFLUCC $12-0270^{\mathrm{T}}$ & dead plant material & China & JX399008 & JX399039 & JX399072 \\
\hline P. intermedia & MFLUCC 12-0259 & dead plant material & China & JX398993 & JX399028 & JX399059 \\
\hline P. italiana & MFLUCC 12-0657 T & Cupressus glabra & Italy & KP781878 & KP781882 & KP781881 \\
\hline P. jester & CBS $109350^{\mathrm{T}}$ & Fagraea bodenii & Papua New Guinea & KM199380 & KM199468 & KM199554 \\
\hline P. jiangxiensis & $\mathrm{LC} 4399^{\mathrm{T}}$ & Camellia sp. & China & KX895009 & KX895341 & KX895227 \\
\hline P. jinchanghensis & $\mathrm{LC} 6636^{\mathrm{T}}$ & Camellia sinensis & China & KX895028 & KX895361 & KX895247 \\
\hline P. kenyana & CBS $442.67^{\mathrm{T}}$ & Coffea sp. & Kenya & KM199302 & KM199395 & KM199502 \\
\hline P. knightiae & CBS $114138^{\mathrm{T}}$ & Knightia sp. & New Zealand & KM199310 & KM199408 & KM199497 \\
\hline
\end{tabular}


Table 2 Continued.

\begin{tabular}{|c|c|c|c|c|c|c|}
\hline Taxa & Strain number a & Host & Country & ITS & $\beta$-tubulin & EF1a \\
\hline P. licualacola & HGUP4057 T & Licuala grandis & China & KC492509 & KC481683 & KC481684 \\
\hline P. linearis & MFLUCC 12-0271 T & dead plant material & China & JX398992 & JX399027 & JX399058 \\
\hline P. longiappendiculata & LC3013 T & Camellia sinensis & China & KX894939 & KX895271 & KX895156 \\
\hline P. lushanensis & LC4344 T & Camellia sp. & China & KX895005 & KX895337 & KX895223 \\
\hline P. macadamiae & BRIP 63738B T & Macadamia sp. & Australia & KX186588 & KX186680 & KX186621 \\
\hline P. malayana & CBS $102220 \mathrm{~T}$ & Macaranga triloba & Malaysia & KM199306 & KM199411 & KM199482 \\
\hline P. monochaeta & CBS $144.97 \mathrm{~T}$ & Quercus robur & Netherlands & KM199327 & KM199386 & KM199479 \\
\hline P. montellica & MFLUCC 12-0279 T & dead plant material & China & JX399012 & JX399043 & JX399076 \\
\hline P. neglecta & TAP1100 T & Quercus myrsinaefolia & Japan & AB482220 & LC311599 & LC311600 \\
\hline P. neolitseae & NTUCC 17-011 T & on leaf of Neolitsea villosa & Taiwan & МH809383 & МH809387 & МH809391 \\
\hline P. novae-hollandiae & CBS $130973 \mathrm{~T}$ & Banksia grandis & Australia & KM199337 & KM199425 & KM199511 \\
\hline P. oryzae & CBS $353.69 \mathrm{~T}$ & Oryza sativa & Denmark & KM199299 & KM199398 & KM199496 \\
\hline P. papuana & CBS $331.96 \mathrm{~T}$ & coastal soil & Papua New Guinea & KM199321 & KM199413 & KM199491 \\
\hline \multirow[t]{2}{*}{ P. parva } & CBS 265.37 T & Delonix regia & - & KM199312 & KM199404 & KM199508 \\
\hline & CBS 278.35 & Leucothoe fontanesiana & - & KM199313 & KM199405 & KM199509 \\
\hline P. pallidotheae & MAFF $240993 \mathrm{~T}$ & Pieris japonica & Japan & NR111022 & LC311584 & LC311585 \\
\hline P. portugalica & CBS $393.48 \mathrm{~T}$ & - & Portugal & KM199335 & KM199422 & KM199510 \\
\hline P. rhododendri & IFRDCC $2399 \mathrm{~T}$ & Rhododendron sinogrande & China & KC537804 & KC537818 & KC537811 \\
\hline P. rhodomyrtus & HGUP4230 T & Rhodomyrtus tomentosa & China & KF412648 & KC537818 & KF412645 \\
\hline P. rosea & MFLUCC 12-0258 T & dead plant material & China & JX399005 & JX399036 & JX399069 \\
\hline P. scoparia & CBS $176.25 \mathrm{~T}$ & Chamaecyparis sp. & - & KM199330 & KM199393 & KM199478 \\
\hline P. shorea & MFLUCC 12-0314 T & dead seed wing of Shorea obtusa & Thailand & KJ503811 & KJ503814 & KJ503817 \\
\hline Pestalotiopsis sp. & LC3637 T & Camellia sp. & China & KX894993 & KX895324 & KX895210 \\
\hline P. spathulata & CBS $356.86 \mathrm{~T}$ & Gevuina avellana & Chile & KM199338 & KM199423 & KM199513 \\
\hline P. telopeae & CBS $114161 \mathrm{~T}$ & Telopea sp. & Australia & KM199296 & KM199403 & KM199500 \\
\hline P. trachicarpicola & IFRDCC $2240 \mathrm{~T}$ & Trachycarpus fortunei & China & NR_120109 & - & - \\
\hline P. unicolor & MFLUCC 12-0276 T & dead plant material & China & JX398999 & JX399030 & - \\
\hline P. verruculosa & MFLUCC 12-0274 T & dead plant material & China & JX398996 & - & JX399061 \\
\hline P. yanglingensis & $\mathrm{LC} 4553 \mathrm{~T}$ & Camellia sinensis & China & KX895012 & KX895345 & KX895231 \\
\hline Pseudopestalotiopsis avucenniae & MFLUCC 17-0434 T & Avicennia marina & Thailand & MK764287 & MK764353 & MK764331 \\
\hline
\end{tabular}


Table 2 Continued.

\begin{tabular}{|c|c|c|c|c|c|c|}
\hline Taxa & Strain number ${ }^{a}$ & Host & Country & ITS & $\beta$-tubulin & EF1a \\
\hline Ps. curvatispora & MFLUCC 17-1722 ${ }^{\mathrm{T}}$ & Rhizophora mucronata & Thailand & MK764288 & MK764354 & MK764332 \\
\hline Ps. curvatispora & MFLUCC 17-1723 & Rhizophora mucronata & Thailand & MK764289 & MK764355 & MK764333 \\
\hline Ps. curvatispora & MFLUCC 17-1747 & Rhizophora mucronata & Thailand & MK764290 & MK764356 & MK764334 \\
\hline Ps. rhizophorae & MFLUCC 17-1560 ${ }^{\mathrm{T}}$ & Rhizophora apiculata & Thailand & MK764291 & MK764357 & MK764335 \\
\hline Ps. thailandica & MFLUCC 17-1724 ${ }^{\mathrm{T}}$ & Rhizophora mucronata & Thailand & MK764292 & MK764358 & MK764336 \\
\hline Ps. thailandica & MFLUCC 17-1725 & Rhizophora mucronata & Thailand & MK764293 & MK764359 & MK764337 \\
\hline Ps. ampullaceae & LC6618 ${ }^{\mathrm{T}}$ & Camellia sinensis & China & KX895025 & KX895358 & KX895244 \\
\hline Ps. camelliae-sinesis & LC3011 ${ }^{\mathrm{T}}$ & Camellia sinensis & China & KX894985 & KX895316 & KX895202 \\
\hline Ps. chinensis & LC $3011^{\mathrm{T}}$ & Camellia sinensis & China & KX894937 & KX895269 & KX895154 \\
\hline Ps. cocos & CBS $272.29^{\mathrm{T}}$ & Cocos nucifera & Java, Indonesia & KM199378 & KM199467 & KM199553 \\
\hline Ps. dawaina & MM14-F0015 ${ }^{\mathrm{T}}$ & unknown & Dawei, Myanmar & LC324750 & LC324751 & LC324752 \\
\hline Ps. ignota & $\mathrm{NN} 42909^{\mathrm{T}}$ & unknown & - & KU500020 & - & KU500016 \\
\hline Ps. indica & CBS $459.78^{\mathrm{T}}$ & Hibiscus rosa-sinensis & - & KM199381 & KM199470 & KM199560 \\
\hline Ps. ixorae & NTUCC $17-001.1^{\mathrm{T}}$ & Ixora sp. & - & MG816316 & MG816326 & MG816336 \\
\hline Ps. jiangxiensis & $\mathrm{LC} 4479^{\mathrm{T}}$ & Eurya sp. & China & KX895034 & KX895343 & KX895229 \\
\hline Ps. kawthaungina & MM14-F0083 ${ }^{\mathrm{T}}$ & unknown & Kawthaung, Myanmar & LC324753 & LC324754 & LC324755 \\
\hline Ps. kubahensis & UMAS KUB-P20 ${ }^{\mathrm{T}}$ & Macaranga sp. & Sarawak, Malaysia & KT006749 & - & - \\
\hline Ps. myanmarina & NBRC $112264^{\mathrm{T}}$ & Averrhoa carambola & Dawei, Myanmar & LC114025 & LC114045 & LC114065 \\
\hline \multirow[t]{2}{*}{ Ps. simitheae } & MFLUCC $12-0121^{\mathrm{T}}$ & Pandanus odoratissimus & Thailand & KJ503812 & KJ503815 & KJ503818 \\
\hline & MFLUCC 12-0125 & living leaves of Pandanus odoratissimus & Thailand & KJ503813 & KJ503816 & KJ503819 \\
\hline \multirow[t]{4}{*}{ Ps. taiwanensis } & NTUCC $17-002.1^{\mathrm{T}}$ & Ixora sp. & Taiwan & MG816319 & MG816329 & MG816339 \\
\hline & NTUCC 17-002.2 & Ixora sp. & Taiwan & MG816320 & MG816330 & MG816340 \\
\hline & NTUCC 17-002.3 & Ixora sp. & Taiwan & MG816321 & MG816331 & MG816341 \\
\hline & NTUCC 17-002.4 & Ixora sp. & Taiwan & MG816322 & MG816332 & MG816342 \\
\hline Ps. theae & MFLUCC $12-0055^{\mathrm{T}}$ & Camellia sinensis & Thailand & JQ683727 & JQ683711 & JQ683743 \\
\hline Ps. vietnamensis & NBRC $112252^{\mathrm{T}}$ & Fragaria sp. & Hue, Vietnam & LC114034 & LC114054 & LC114074 \\
\hline
\end{tabular}

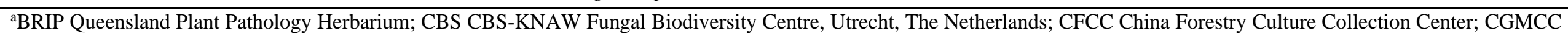

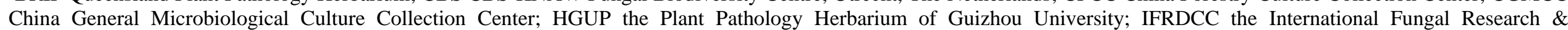

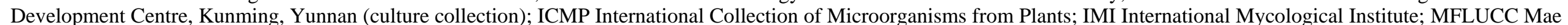

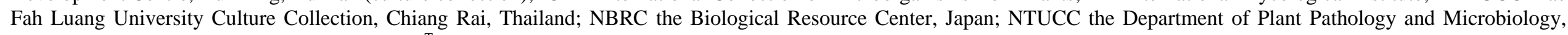
National Taiwan University Culture Collection. ${ }^{\mathrm{T}}$ Ex-type strains. Strains in this study are in bold. 


\section{Results}

\section{Phylogenetic analysis of combined ITS, $\beta$-tubulin and EF1 $\alpha$ sequences}

The results from the partition homogeneity tests (PHT) for all three phylogenetic trees were not significant (level $\leq 95 \%$ ), indicating that the individual datasets were congruent and could be combined. The combined alignment of the three phylogeny analyses trees included 22 strains, of which 11 strains belong to Neopestalotiopsis, four strains belong to Pestalotiopsis and seven strains belong to Pseudopestalotiopsis.

The alignment of Neopestalotiopsis (Fig. 1) comprised 52 taxa, with the outgroup taxon, Pestalotiopsis diversiseta (MFLUCC 12-0287). The total length of the dataset was 1358 characters including alignment gaps, 1-324, 325-482, 483-700, 701-886, 887-1054 and 1055-1358 corresponding to ITS1+ ITS2, 5.8S, $\beta$-tubulin (exon), $\beta$-tubulin (intron), EF1 $\alpha$ (exon) and EF1 $\alpha$ (intron), respectively. The combined dataset contained 1001 constant, 175 parsimony uninformative and 182 parsimony informative characters. The combined dataset was analyzed using MP, ML and BI. The trees generated under different optimality criteria were essentially similar in topology and did not differ significantly (data not shown). The descriptive statistics generated from MP analysis based on the combined dataset of ITS1+ITS2, 5.8S, $\beta$-tubulin (exon), $\beta$-tubulin (intron), EF1 $\alpha$ (exon), and EF1 $\alpha$ (intron) were $\mathrm{TL}=643, \mathrm{CI}=0.673, \mathrm{RI}=0.670, \mathrm{RC}=$ $0.451, \mathrm{HI}=0.327$. The best scoring likelihood tree selected with a final value for the combined dataset $=-5197.543923$.

Neopestalotiopsis acrostichi Norphanphoun, T.C. Wen \& K.D. Hyde, sp. nov.

Fig. 2

Index Fungorum number: IF556434; Facesoffungi number: FoF 05780

Etymology - refers to the host from which the fungus was isolated, Acrostichum aureum L.

Holotype - MFLU 19-0774

Associated with leaf spots of Acrostichum aureum L. Symptoms sub circular to irregular shape, pale brown, appear on adaxial surface leaves of A. aureum, which later expand outwards (Fig. 2c). Small brown spots appeared initially and then gradually enlarged, changing to pale brown circular spots with a black border. They were usually 4-5 circular spots occurred on a single affected leaf (Fig. 2b).Asexual morph: Conidiomata pycnidial, globose, brown, semi-immersed on PDA, releasing conidia in a black, slimy, globose, glistening mass. Conidiophores indistinct. Conidiogenous cells discrete to lageniform, hyaline, smooth, thin-walled, proliferating once percurrently, collarette present and not flared, 10-25 × 2-5 $\mu \mathrm{m}$. Conidia (22-)23-26(-27) $\times(5-$ )5.5-6.5(-7) $\mu \mathrm{m}$, (mean $\pm \mathrm{SD}=24.3 \pm 1.3 \times 6 \pm 0.6 \mu \mathrm{m}$ ), fusiform to clavate, straight to slightly curved, 4-septate; basal cell obconic with a truncate base, hyaline or sometimes pale brown, thinand smooth-walled, $(4.5-) 5-6(-7) \mu \mathrm{m}$ long (mean $\pm \mathrm{SD}=5.5 \pm 0.8 \mu \mathrm{m})$; three median cells $(14-$ )15-16(-18) $\mu \mathrm{m}$ long (mean $\pm \mathrm{SD}=15.9 \pm 1 \mu \mathrm{m})$, brown, septa and periclinal walls darker than rest of the cell, versicolored, wall rugose; second cell from base pale brown, $(4.5-) 5-6(-6.5) \mu \mathrm{m}$ long (mean $\pm \mathrm{SD}=5.6 \pm 0.6 \mu \mathrm{m})$; third cell brown, $4.5-6 \mu \mathrm{m}$ long $($ mean $\pm \mathrm{SD}=4.9 \pm 0.5 \mu \mathrm{m})$; fourth cell brown, (4-)4.5-5(-6.5) $\mu \mathrm{m}$ long (mean $\pm \mathrm{SD}=5.4 \pm 0.6 \mu \mathrm{m})$; apical cell (3-)4.5-5(-6) $\mu \mathrm{m}$ long (mean $\pm \mathrm{SD}=4.9 \pm 0.8 \mu \mathrm{m}$ ), hyaline, conic to acute, with $3-5$ tubular appendages on apical cell, inserted at different loci but in a crest at the apex of the apical cell, unbranched, flexuous, (16-)19-28.5(-33.5) $\mu \mathrm{m}$ long (mean $\pm \mathrm{SD}=24.5 \pm 4.7 \mu \mathrm{m})$; single basal appendage, tubular, unbranched, centric, (4.5-)5-7(-12) $\mu \mathrm{m}$ long, (mean $\pm \mathrm{SD}=7.1 \pm 2 \mu \mathrm{m})$.

Culture characteristics - Colonies on PDA reaching 6-8 $\mathrm{cm}$ diam after $7 \mathrm{~d}$ at room temperature $\left( \pm 25^{\circ} \mathrm{C}\right)$, under light $12 \mathrm{hr} /$ dark $12 \mathrm{hr}$, colonies filamentous to circular, medium dense, aerial mycelium on surface flat or raised, with filiform margin (curled margin), fluffy, white from above and reverse; fruiting bodies black.

Material examined - THAILAND, Chanthaburi Province, leaf spots of Acrostichum aureum L., 25 April 2017, Norphanphoun Chada JT12-1 (MFLU 19-0774 dried culture, holotype; PDD, 


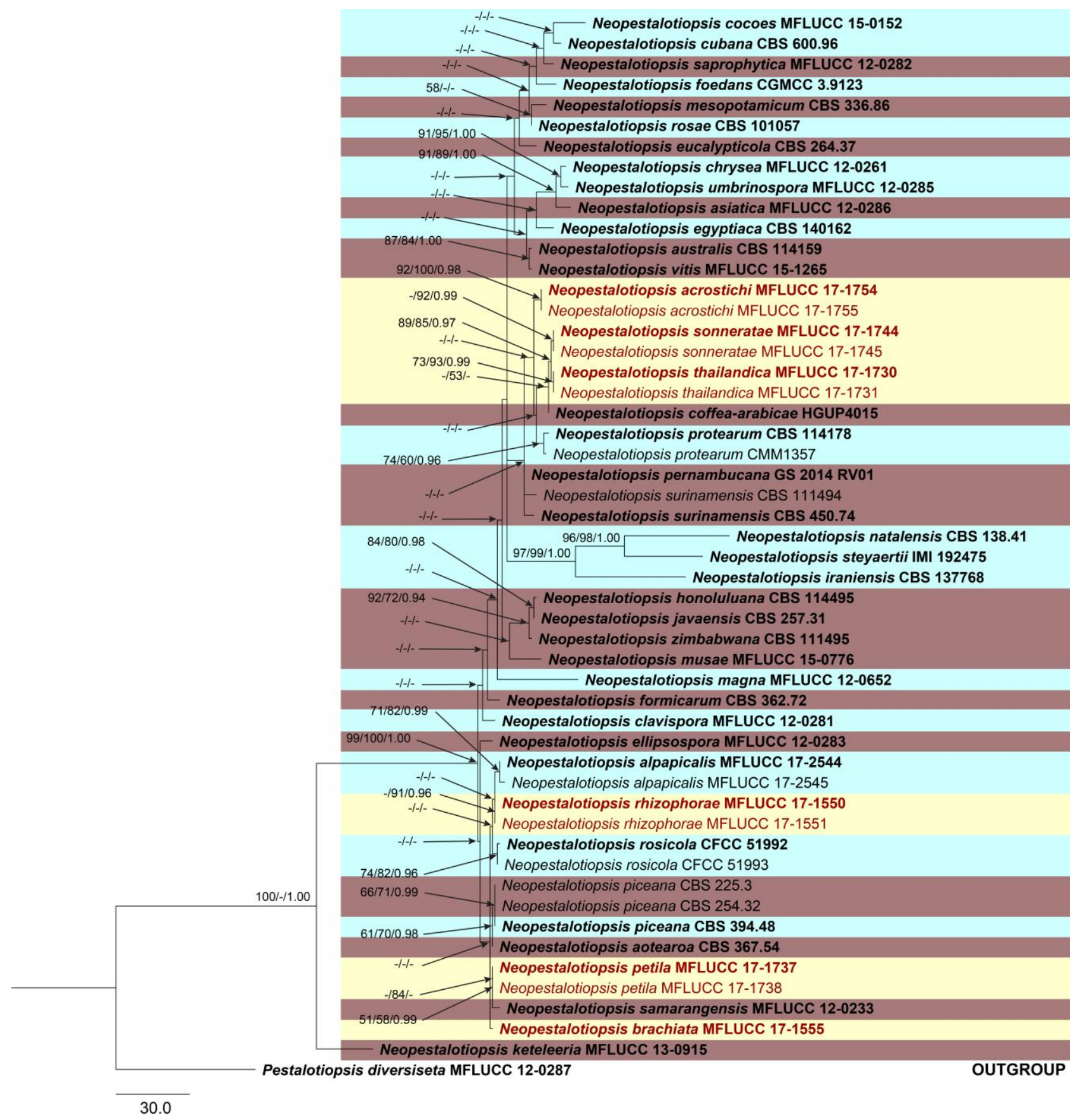

Figure 1 - One of the 1000 most parsimonious trees obtained from a heuristic search of combined ITS, $\beta$-tubulin and EF1 $\alpha$ sequence data for the genus Neopestalotiopsis. The tree is rooted to Pestalotiopsis diversiseta (MFLUCC 12-0287). Maximum parsimony and maximum likelihood bootstrap values $\leq 50 \%$, Bayesian posterior probabilities $\leq 0.90$ (MPBS/MLBS/PPBY) are given at the nodes. The species obtained in this study are in red. Ex-type taxa from other studies are in black bold.

isotype); ex-type-living cultures, MFLUCC 17-1754, TNCC. THAILAND, Chanthaburi Province, leaf spots of Acrostichum aureum L., 25 April 2017, Norphanphoun Chada JT12-2 (MFLU 190775 dried culture, paratype); ex-type-living cultures, MFLUCC 17-1755.

Notes - Neopestalotiopsis acrostichi was isolated from a leaf spot of Acrostichum aureum. The new species resembles several other Neopestalotiopsis species. The combined phylogenetic tree indicated that $N$. acrostichi is sister to $N$. protearum (CBS 114178) and $N$. surinamensis (CBS 450.74) (Fig. 1), but they differ in morphology. Neopestalotiopsis acrostichi have larger conidia than $N$. protearum $(N$. protearum: $(14-) 16-17(-18) \times(6.5-) 8-9(-10))$. Neopestalotiopsis acrostichi is very much similar to $N$. surinamensis (Crous et al. 2011, Maharachchikumbura et al. 
2014). However, $N$. acrostichi differs from this species by having smaller conidia (N. surinamensis: (23-)24-28(-29) $\times(7-) 7.5-9(-9.5))$ with four apical tubular appendages (N. surinamensis: (15)18-27(-28)) (Table 3, Maharachchikumbura et al. 2014). This is the first report of Neopestalotiopsis on A. aureum.

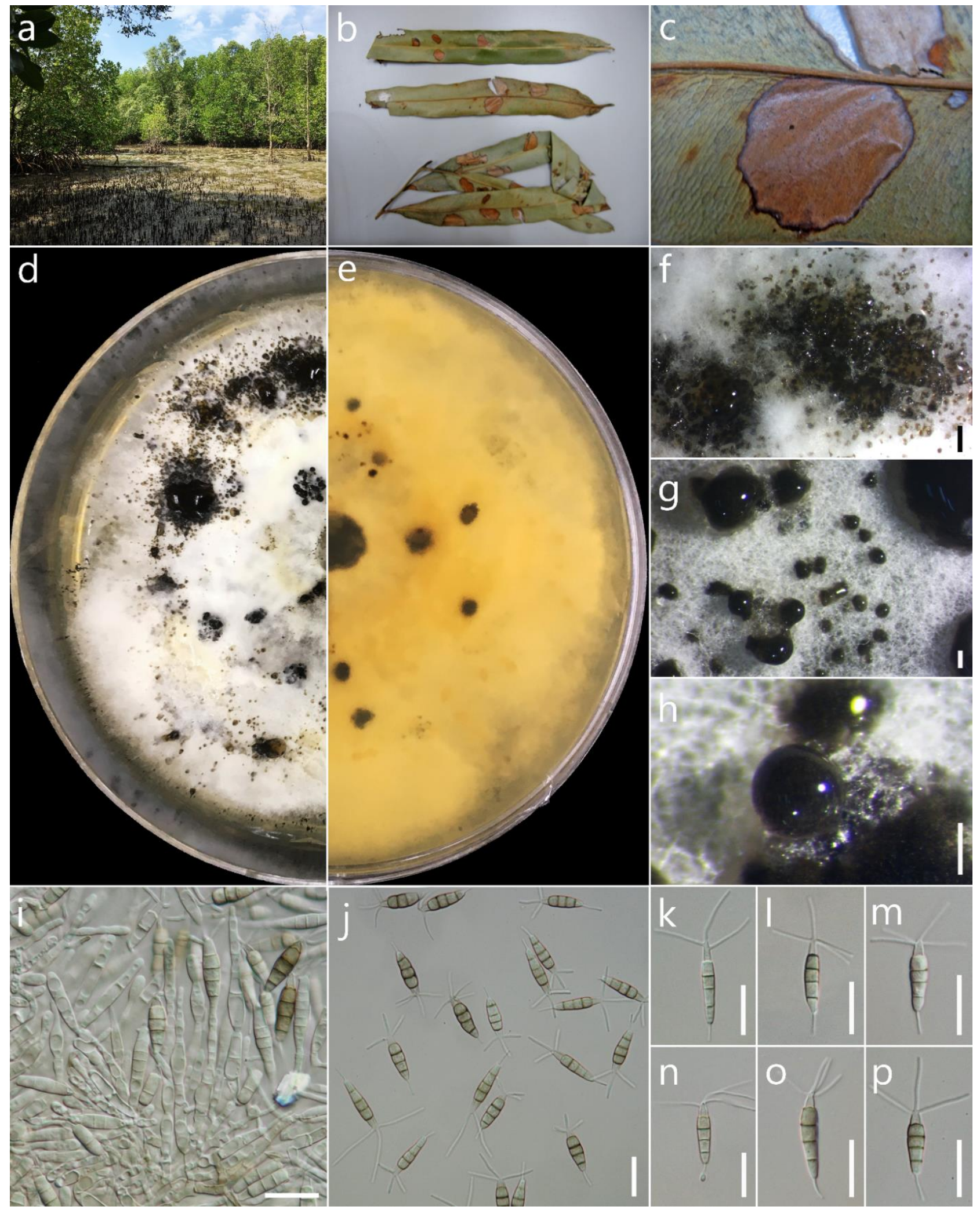

Figure 2 - Neopestalotiopsis acrostichi (MFLU 19-0774, holotype). a Habitat. b, c Leaf spots on Acrostichum aureum. $\mathrm{d}$, e Culture on PDA (d-above, e-reverse). $\mathrm{f}-\mathrm{h}$ Colony sporulating on PDA. $\mathrm{i}$ Conidiogenous cells giving rise to conidia. $\mathrm{j}-\mathrm{p}$ Conidia. Scale bars: $\mathrm{f}-\mathrm{h}=200 \mu \mathrm{m}, \mathrm{i}-\mathrm{p}=20 \mu \mathrm{m}$. 
Neopestalotiopsis brachiata Norphanphoun, T.C. Wen \& K.D. Hyde, sp. nov.

Index Fungorum number: IF556435; Facesoffungi number: FoF 05771

Etymology - the specific epithet "brachiata" refers to the character of apical appendages (Latin 'brachiata' means 'branches').

Holotype - MFLU 19-0776

Associated with leaf spots of Rhizophora apiculata Blume. Symptoms circular or sub circular shape, grayish brown, slightly sunken spots appear on adaxial surface leaves of $R$. apiculata, which later expand outwards (Fig. 3c). Small auburn spots appeared initially and then gradually enlarged, changing to grayish brown circular ring spots with a dark brown border. They were usually $>5$ circulars, which occurred on a single affected leaf. In severe cases, lesions spreaded evenly on the leaves (Fig. 3b). Asexual morph: Conidiomata pycnidial, globose, brown, semi-immersed on PDA, releasing conidia in a black, slimy, globose, glistening mass. Conidiophores indistinct. Conidiogenous cells discrete to ampulliform to lageniform, hyaline, smooth- and thin-walled, simple, proliferating 1-2 times percurrently, collarette present and not flared, 5-10 $\times 5-8 \mu \mathrm{m}$. Conidia (18-)18.5-25(-26) $\times(5-) 5.5-6(-6.5) \mu \mathrm{m}$ (mean $\pm \mathrm{SD}=21 \pm 2.2 \times 5.5 \pm 0.6 \mu \mathrm{m}$ ), fusiform to clavate, straight to slightly curved, 4-septate; basal cell obconic with a truncate base, hyaline or sometimes pale brown, thin- and smooth-walled, (3-)3.5-4(-5) $\mu \mathrm{m}$ long (mean $\pm \mathrm{SD}=3.8 \pm 0.7$ $\mu \mathrm{m}$ ); three median cells (10-)10.5-15(-16) $\mu \mathrm{m}$ long (mean $\pm \mathrm{SD}=12.7 \pm 1.7 \mu \mathrm{m}$ ), brown, septa and periclinal walls darker than rest of the cell, versicolored, wall rugose; second cell from base pale brown, (3-)4-5(-6) $\mu \mathrm{m}$ long (mean $\pm \mathrm{SD}=4.4 \pm 0.8 \mu \mathrm{m}$ ); third cell brown, $(3.5-) 4-5(-6) \mu \mathrm{m}$ long (mean $\pm \mathrm{SD}=4.2 \pm 0.7 \mu \mathrm{m}$ ); fourth cell brown, $(3-) 4-5(-5.5) \mu \mathrm{m}$ long (mean $\pm \mathrm{SD}=4.1 \pm$ $0.5 \mu \mathrm{m}$ ); apical cell (4-)4.5-5(-6) $\mu \mathrm{m}$ long (mean $\pm \mathrm{SD}=4.5 \pm 0.7 \mu \mathrm{m})$, hyaline, conic to acute; with 1-3 tubular appendages on apical cell, inserted at different loci in a crest at the apex of the apical cell, branched, flexuous, (8.5-)9.5-33(-34) $\mu \mathrm{m}$ long (mean $\pm \mathrm{SD}=20 \pm 7.6 \mu \mathrm{m}$ ); single basal appendage, tubular, unbranched, centric, $(3.5-) 4-9(-10) \mu \mathrm{m}$ long (mean $\pm \mathrm{SD}=6.5 \pm 1.6 \mu \mathrm{m})$.

Culture characteristics - Colonies on PDA reaching 6-7 cm diam after $7 \mathrm{~d}$ at room temperature $\left( \pm 25^{\circ} \mathrm{C}\right)$, under light $12 \mathrm{hr} /$ dark $12 \mathrm{hr}$, colonies filamentous to circular, medium dense, aerial mycelium on surface flat or raised, with filiform margin (curled margin), fluffy, white from above and reverse; fruiting bodies black.

Material examined - THAILAND, Ngao, Ranong Province, Ngao Mangrove Forest Research Centre, leaf spots of Rhizophora apiculata, 6 December 2016, Norphanphoun Chada NG33 (MFLU 19-0776 dried culture, holotype; PDD, isotype); ex-type-living cultures, MFLUCC 17-1555, TNCC.

Notes - Neopestalotiopsis brachiata is similar to N. rosicola (strain CFCC 51992) in conidial size $(N$. brachiata $(18-) 18.5-25(-26) \times(5-) 5.5-6(-6.5) \mu \mathrm{m} v$ s. $N$. rosicola: $(19-) 20-25.5(-26) \times$ $(5-) 5.5-8(-8.5) \mu \mathrm{m})$ but phylogenetically distinct (Jiang et al. 2018). In the multigene phylogenetic analysis presented here, the new species formed a sister clade to N. aotearoa (strain CBS 367.54), $N$. piceanae (strain CBS 394.48) and $N$. petila (in this study), which appear to be phylogenetically distinct (Fig. 1). The conidia of N. brachiata are different from N. aotearoa $((19.5-) 21-28(-29) \times$ $(6-) 6.5-8.5(-9) \mu \mathrm{m}), N$. piceanae $((19-)) 19.5-25(-26) \times(7-) 7.5-9(-9.5) \mu \mathrm{m})$ and $N$. petila $((20-$ 21-26.5(-27.5) $\times(5.5-) 6-7(-8) \mu \mathrm{m})$ and also differs by having branched, flexuous apical tubular appendages (Maharachchikumbura et al. 2014).

Neopestalotiopsis petila Norphanphoun, T.C. Wen \& K.D. Hyde, sp. nov.

Fig. 4

Index Fungorum number: IF556436; Facesoffungi number: FoF 05772

Etymology - the latin "petilus" meaning slander/slim, which refers to the shape of conidia.

Holotype - MFLU 19-0777

Associated with leaf spots of Rhizophora mucronata Lam. Symptoms subcircular to the irregular shape, pale brown, slightly sunken spots appear on adaxial surface leaves of $R$. mucronata, which later expand outwards (Fig. 4c). Small auburn spots appeared initially and then gradually enlarged, changing to pale-auburn circular ring spots with a dark auburn border. 


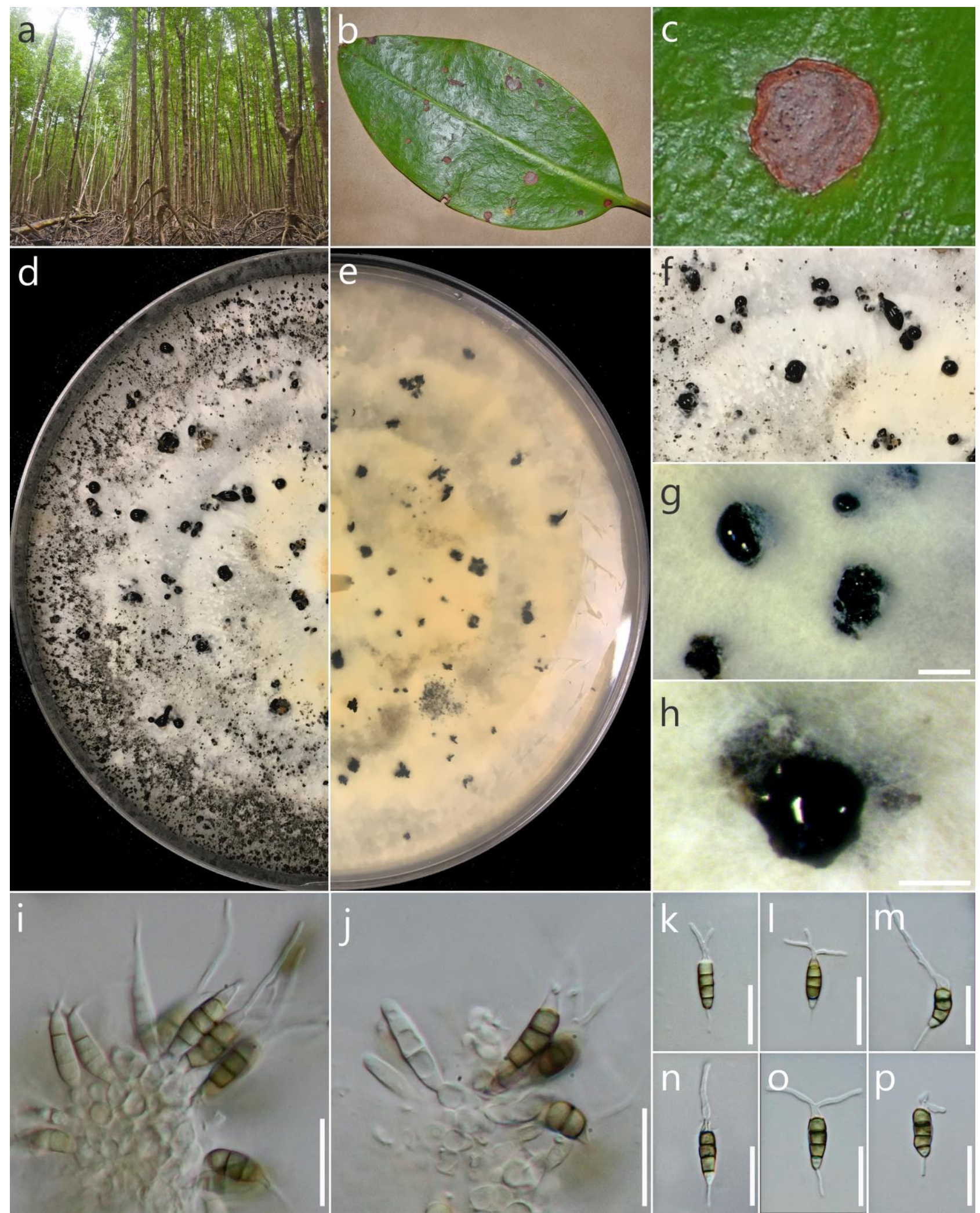

Figure 3 - Neopestalotiopsis brachiata (MFLU 19-0776, holotype). a Habitat. b, c Leaf spots on Rhizophora apiculata. d, e Culture on PDA (d-above, e-reverse). $\mathrm{f}-\mathrm{h}$ Colony sporulating on PDA. i Conidiogenous cells giving rise to conidia. $\mathrm{j}-\mathrm{p}$ Conidia. Scale bars: $\mathrm{g}=1000 \mu \mathrm{m}, \mathrm{h}=500 \mu \mathrm{m}$, $\mathrm{i}-\mathrm{p}=20 \mu \mathrm{m}$.

They were usually $>5$ circulars, which occurred on a single affected leaf. In severe cases, lesions spread evenly on the leaves (Fig. 4b). Asexual morph: Conidiomata pycnidial, globose, brown, semi-immersed on PDA, releasing conidia in a black, slimy, globose, glistening mass. 
Conidiophores distinct. Conidiogenous cells discrete to integrated, hyaline, smooth- and thinwalled, proliferating one time percurrently, collarette present and not flared. Conidia (20-)21$26.5(-27.5) \times(5.5-) 6-7(-8) \mu \mathrm{m}($ mean $\pm \mathrm{SD}=24.5 \pm 2.0 \times 6.7 \pm 0.7 \mu \mathrm{m})$, fusiform to clavate, straight to slightly curved, 4-septate; basal cell obconic with a truncate base, hyaline or sometimes pale brown, thin- and smooth-walled, (3-)4-5.5(-6) $\mu \mathrm{m}$ long (mean $\pm \mathrm{SD}=4.52 \pm 0.8 \mu \mathrm{m}$ ); three median cells $(12.5-) 13.5-15(-17) \mu \mathrm{m}$ long (mean $\pm \mathrm{SD}=15.3 \pm 1.0 \mu \mathrm{m})$, brown, septa and periclinal walls darker than rest of the cell, versicolored, wall rugose; second cell from base pale brown, (4.5-)5-6(-7) $\mu \mathrm{m}$ long (mean $\pm \mathrm{SD}=5.2 \pm 0.5 \mu \mathrm{m})$; third cell brown, $(3.5-) 4-5(-5.5) \mu \mathrm{m}$ long (mean $\pm \mathrm{SD}=4.8 \pm 0.5 \mu \mathrm{m}$ ); fourth cell brown, $(4.5-) 5-5.5(-6) \mu \mathrm{m}$ long (mean $\pm \mathrm{SD}=5.3 \pm$ $0.5 \mu \mathrm{m}$ ); apical cell (3-)4-5(-7) $\mu \mathrm{m}$ long (mean $\pm \mathrm{SD}=4.6 \pm 1.0 \mu \mathrm{m})$, hyaline, conic to acute; with 2-3 tubular appendages on apical cell, inserted at different loci but in a crest at the apex of the apical cell, unbranched, flexuous, $(21-) 22-29(-33) \mu \mathrm{m}$ long (mean $\pm \mathrm{SD}=25.5 \pm 3.1 \mu \mathrm{m})$; single basal appendage, tubular, unbranched, centric, $(2-) 3-8(-9) \mu \mathrm{m}$ long (mean $\pm \mathrm{SD}=6 \pm 2.1 \mu \mathrm{m}$ ).

Culture characteristics - Colonies on PDA reaching 5-6 cm diam after $7 \mathrm{~d}$ at room temperature $\left( \pm 25^{\circ} \mathrm{C}\right)$, under light $12 \mathrm{hr} /$ dark $12 \mathrm{hr}$, colonies filamentous to circular, medium dense, aerial mycelium on surface flat or raised, with filiform margin (curled margin), fluffy, white from above and reverse; fruiting bodies black.

Material examined - THAILAND, Kor Chang, Trat Province, leaf spots of Rhizophora mucronata, 27 April 2017, Norphanphoun Chada KC05-1 (MFLU 19-0777 dried culture, holotype; PDD, isotype); ex-type-living cultures, MFLUCC 17-1737, TNCC. THAILAND, Kor Chang, Trat Province, leaf spots of $R$. mucronata, 27 April 2017, Norphanphoun Chada KC05-2 (MFLU 190778 dried culture, paratype); living cultures, MFLUCC 17-1738.

Notes - Based on multigene analyses, Neopestalotiopsis petila is closely related to $N$. aotearoa (strain CBS 367.54), $N$. piceana (strain CBS 394.48), $N$. brachiata (in this study) and $N$. samarangensis (strain MFLUCC 12-0233) (Fig. 1). However, N. petila differs from N. aotearoa in having long apical appendages (N. petila: $(21-) 22-29(-33)$ vs. N. aotearoa: $(3-) 5-12(-13))$ and differs from $N$. piceana by having 2-3 apical appendages ( $N$. piceana containing only 3 appendages) and short basal appendage (N. petila: (2-)3-8(-9) vs. N. piceana: 6-23) and larger conidia than $N$. samarangensis $(N$. petila: $(20-) 21-26.5(-27.5) \times(5.6-) 6-7(-7.8) \quad v s . \quad N$. samarangensis: 18-21 × 6.5-7.5) (Maharachchikumbura et al. 2014, 2013). The morphological differences between $N$. petila and $N$. brachiata have been mentioned above as notes of $N$. brachiata. Thus, $N$. petila is considered a novel species.

Neopestalotiopsis rhizophorae Norphanphoun, T.C. Wen \& K.D. Hyde, sp. nov.

Fig. 5

Index Fungorum number: IF556437; Facesoffungi number: FoF 05773

Etymology - refers to the host from which the fungus was collected, Rhizophora mucronata Lam.

Holotype - MFLU 19-0779

Associated with leaf spots of Rhizophora mucronata Lam. Symptoms irregular to subcircular shape, brown, slightly sunken spots appear on adaxial surface leaves of $R$. mucronata, which later expand outwards on the surface of the leaves (Fig. 5c). Small auburn spots appeared initially and then gradually enlarged, changing to tawny circular ring spots with a dark mahogany border and jagged edge. They were usually $>5$ circulars, which occurred on a single affected leaf. In severe cases, lesions spread evenly on the leaves (Fig. 5b). Asexual morph: Conidiomata pycnidial, globose, brown, semi-immersed on PDA releasing conidia in a black, slimy, globose, glistening mass. Conidiophores distinct. Conidiogenous cells discrete or integrated, ampulliform, clavate or

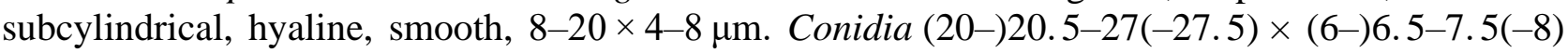
$\mu \mathrm{m}($ mean $\pm \mathrm{SD}=24.5 \pm 0.3 \times 7.2 \pm 0.6 \mu \mathrm{m})$, fusiform to clavate, straight to slightly curved, 4septate; basal cell obconic with a truncate base, hyaline or sometimes pale brown, thin- and smooth-walled, $(2-) 3-4(-5) \mu \mathrm{m}$ long (mean $\pm \mathrm{SD}=3.7 \pm 0.6 \mu \mathrm{m})$; three median cells (14-)15.5$17(-19.5) \mu \mathrm{m}$ long (mean $\pm \mathrm{SD}=16.7 \pm 1.4 \mu \mathrm{m})$, brown, septa and periclinal walls darker than rest 


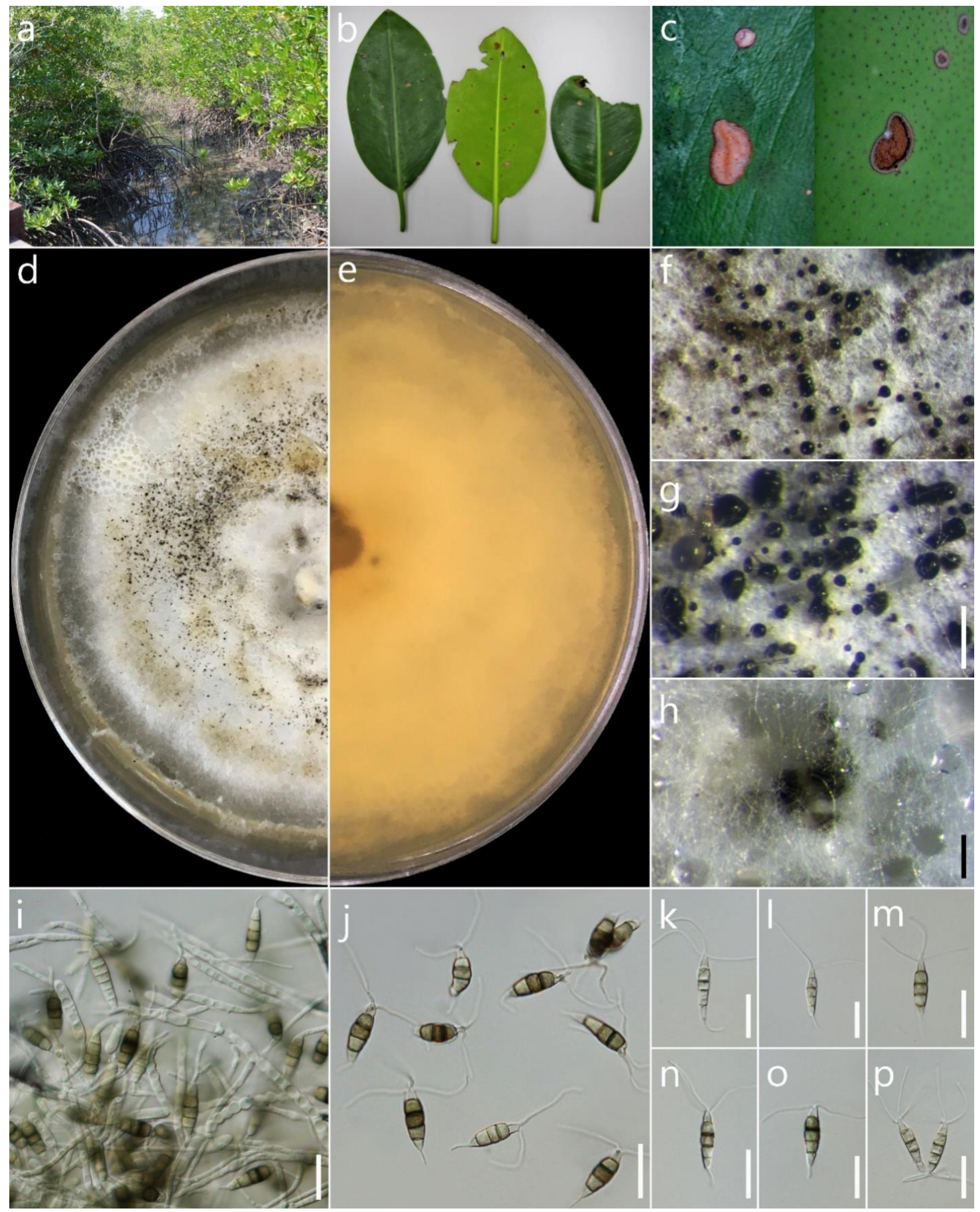

Figure 4 - Neopestalotiopsis petila (MFLU 19-0777, holotype). a Habitat. b, c Leaf spots on Rhizophora mucronata. $\mathrm{d}$, e Culture on PDA (d-above, e-reverse). $\mathrm{f}-\mathrm{h}$ Colony sporulating on PDA. i Conidiogenous cells giving rise to conidia. $\mathrm{j}-\mathrm{p}$ Conidia. Scale bars: $\mathrm{g}-\mathrm{h}=200 \mu \mathrm{m}, \mathrm{i}-\mathrm{p}=20 \mu \mathrm{m}$.

of the cell, versicolored, wall rugose; second cell from base pale brown, (4.5-)5-6(-7) $\mu \mathrm{m}$ long (mean $\pm \mathrm{SD}=5.9 \pm 0.7 \mu \mathrm{m}$ ); third cell brown, $(4-) 5-7(-10) \mu \mathrm{m}$ long (mean $\pm \mathrm{SD}=5.2 \pm 0.6 \mu \mathrm{m}$ ); fourth cell brown, $(4-) 5-5.5(-6.5) \mu \mathrm{m}$ long (mean $\pm \mathrm{SD}=5.5 \pm 0.6 \mu \mathrm{m})$; apical cell $(3-) 3.5-4(-$ 5.5) $\mu \mathrm{m}$ long (mean $\pm \mathrm{SD}=4.1 \pm 0.7 \mu \mathrm{m}$ ), hyaline, conic to acute; with 2-3 tubular appendages on 
apical cell, inserted at different loci but in a crest at the apex of the apical cell, unbranched, flexuous, (6-)12.5-22(-24) $\mu \mathrm{m}$ long (mean $\pm \mathrm{SD}=17.5 \pm 4.4 \mu \mathrm{m}$ ); single basal appendage, tubular, unbranched, centric, with or without knob (2.5-)3-9.5(-10) $\mu \mathrm{m}$ long (mean $\pm \mathrm{SD}=5.2 \pm$ $1.8 \mu \mathrm{m})$.

Culture characteristics - Colonies on PDA reaching 7-8 $\mathrm{cm}$ diam after $7 \mathrm{~d}$ at room temperature $\left( \pm 25^{\circ} \mathrm{C}\right)$, under light $12 \mathrm{hr} /$ dark $12 \mathrm{hr}$, colonies filamentous to circular, medium dense, aerial mycelium on surface flat or raised, with filiform margin (curled margin), fluffy, white from above and reverse; fruiting bodies black.

Material examined - THAILAND, Ngao, Ranong Province, Ngao Mangrove Forest Research Centre, leaf spots of Rhizophora mucronata, 6 December 2016, Norphanphoun Chada NG16a (MFLU 19-0779 dried culture, holotype; PDD, isotype); ex-type-living cultures, MFLUCC 171550, TNCC. THAILAND, Ngao, Ranong Province, Ngao Mangrove Forest Research Centre, leaf spots of Rhizophora mucronata, 6 December 2016, Norphanphoun Chada NG16b (MFLU 19-0780 dried culture, paratype); ex-type-living cultures, MFLUCC 17-1551.

Notes - The new species Neopestalotiopsis rhizophorae (MFLUCC 17-1550, MFLUCC 17$1551)$ is isolated from $R$. mucronata, from Ranong province in Thailand. Neopestalotiopsis rhizophorae is most similar to $N$. petila and $N$. thailandica (this study) in its conidial size (N. petila: $(20-) 21-26.5(-27.5) \times(5.5-) 6-7(-8) \mu \mathrm{m}, N$. thailandica: $(20-) 21-25(-25.5) \times(5.5-) 6-7(-7.5)$ $\mu \mathrm{m})$ (Table 3). However, based on combined gene phylogenetic analysis, $N$. rhizophorae is separated from $N$. petila and $N$. thailandica, and nested between these two species; $N$. alpapicalis (MFLUCC 17-2544, MFLUCC 17-2545), the collections from $R$. mucronata in Phuket, Thailand and $N$. rosicola (strain CFCC 51992, CFCC 51993), the collections from Rosa chinensis in China (Fig. 1). Neopestalotiopsis rhizophorae can be distinguished from $N$. alpapicalis by larger conidia and longer appendages $(N$. alpapicalis: conidial size 14-22.5 $\times 5-7 \mu \mathrm{m}$, apical appendages 5.5-15 $\mu \mathrm{m}$, basal appendages 3-6.5 $\mu \mathrm{m}$, Table 3 ) with polymorphic nucleotide differences of $\beta$-tubulin (5bp) and EF1 $\alpha$ (3-bp) sequence data. It differs from $N$. rosicola by ITS (4-bp) and EF1 $\alpha$ (4-bp) sequence data.

Neopestalotiopsis sonneratae Norphanphoun, T.C. Wen \& K.D. Hyde, sp. nov.

Fig. 6

Index Fungorum number: IF556438; Facesoffungi number: FoF 05774

Etymology - refers to the host from which the fungus was isolated, Sonneronata alba L.

Holotype - MFLU 19-0781

Associated with leaf spots on Sonneronata alba L. Symptoms small circular shape, auburn, slightly spots appear on adaxial surface leaves of $S$. alba, which later expand outwards on the surface of the leaves (Fig. 6c). Small auburn spots appeared initially and then gradually enlarged, changing to dark brown circular spots and border with blurred edge. They were usually $>5$ circulars, which occurred on a single affected leaf (Fig. 6b). Asexual morph: Conidiomata pycnidial, 200-400 $\mu \mathrm{m}$ diam, globose, brown, semi-immersed on PDA, releasing conidia in a black, slimy, globose, glistening mass. Conidiophores indistinct. Conidiogenous cells discrete to lageniform, hyaline, smooth- and thin-walled, 3-8 $\times 2-6 \mu \mathrm{m}$, proliferating one time percurrently, collarette present and not flared. Conidia (21.5-)24-26(-28) $\times 7-7.5(-8)$, (mean \pm SD $=24 \pm 1.6 \times$ $7.51 \pm 0.4 \mu \mathrm{m}$ ), fusiform to clavate, straight to slightly curved, 4-septate; basal cell obconic with a truncate base, hyaline or sometimes pale brown, thin- and smooth-walled, (2-)3-3.5(-4) $\mu \mathrm{m}$ long (mean $\pm \mathrm{SD}=3 \pm 0.6$ ); three median cells $(14.5-) 15-16.5(-17.5) \mu \mathrm{m}$ long (mean $\pm \mathrm{SD}=15.8 \pm$ $0.9)$, brown, septa and periclinal walls darker than rest of the cell, versicolored, wall rugose; second cell from base pale brown, $(4.5-) 5-6(-7) \mu \mathrm{m}$ long (mean $\pm \mathrm{SD}=5.6 \pm 0.8 \mu \mathrm{m}$ ); third cell brown, (4-)5-5.5(-6) $\mu \mathrm{m}$ long (mean $\pm \mathrm{SD}=5.2 \pm 0.7 \mu \mathrm{m}$ ); fourth cell brown, (4-)5-6(-7) $\mu \mathrm{m}$ long (mean $\pm \mathrm{SD}=5.1 \pm 0.7 \mu \mathrm{m}$ ); apical cell (3.5-)4-4.5(-5) $\mu \mathrm{m}$ long (mean $\pm \mathrm{SD}=4 \pm 0.6 \mu \mathrm{m}$ ), hyaline, conic to acute; with 1-3 tubular appendages on apical cell, inserted at different loci but in a crest at the apex of the apical cell, unbranched, flexuous, (5.5-)7-8(-14) $\mu \mathrm{m}$ long (mean $\pm \mathrm{SD}=8.5 \pm 2$ ); single basal appendage, tubular, unbranched, centric, $(2.5-) 3-4(-5) \mu \mathrm{m}$ long (mean $\pm \mathrm{SD}=3.4 \pm$ $0.9)$ long. 


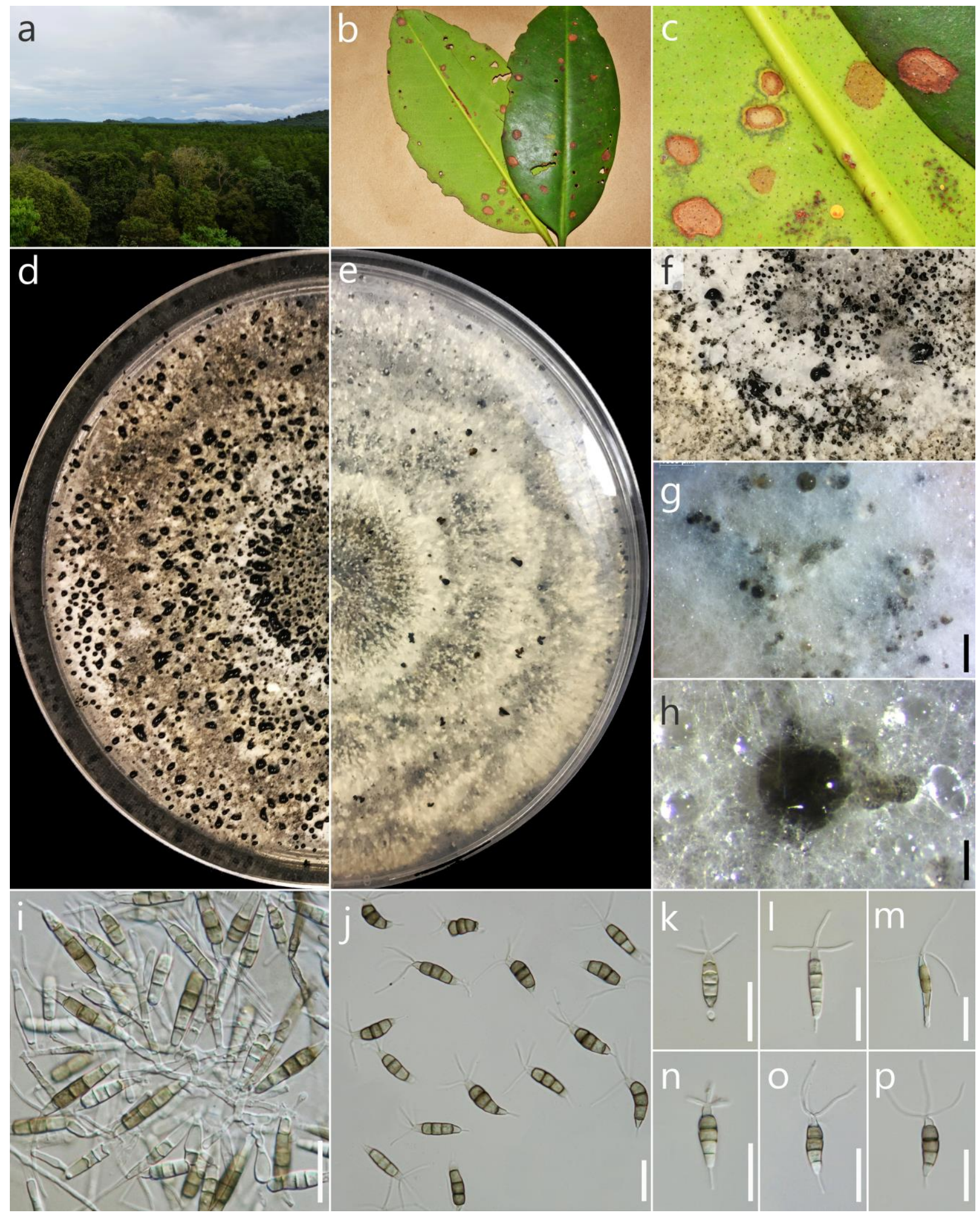

Figure 5 - Neopestalotiopsis rhizophorae (MFLU 19-0779, holotype). a Habitat. b, c Leaf spots on Rhizophora mucronata. d, e Culture on PDA (d-above, e-reverse). $\mathrm{f}-\mathrm{h}$ Colony sporulating on PDA. $\mathrm{i}$ Conidiogenous cells giving rise to conidia. $\mathrm{j}-\mathrm{p}$ Conidia. Scale bars: $\mathrm{g}=1000 \mu \mathrm{m}, \mathrm{h}=100 \mu \mathrm{m}, \mathrm{i}=$ $50 \mu \mathrm{m}, \mathrm{j}-\mathrm{p}=20 \mu \mathrm{m}$.

Culture characteristics - Colonies on PDA reaching 5-6 cm diam after $7 \mathrm{~d}$ at room temperature $\left( \pm 25^{\circ} \mathrm{C}\right)$, under light $12 \mathrm{hr} /$ dark $12 \mathrm{hr}$, colonies filamentous to circular, medium dense, aerial 
mycelium on surface flat or raised, with filiform margin (curled margin), moderate-to-strongly fluffy, fluffy to floccose, white from above and reverse; fruiting bodies black.

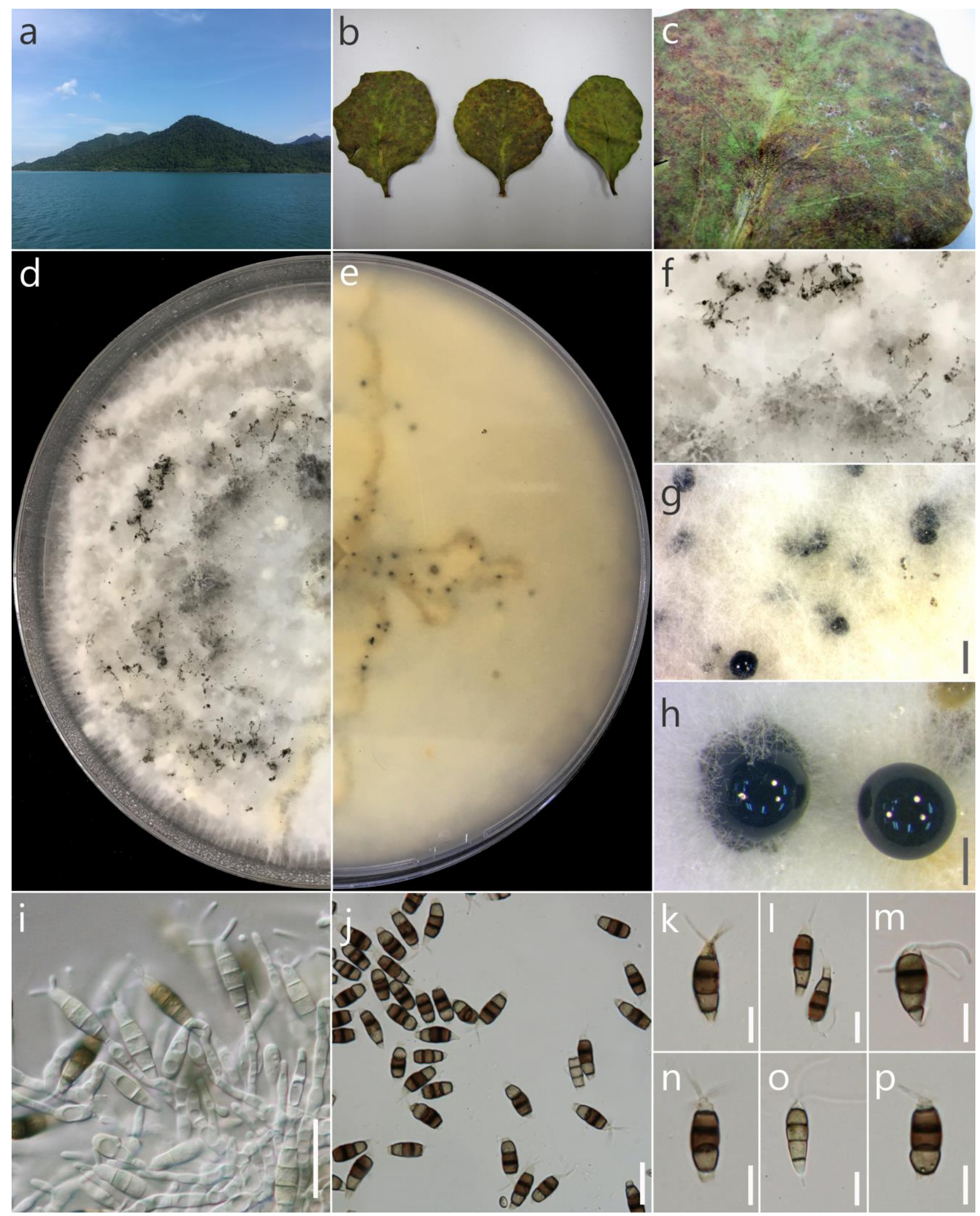

Figure 6 - Neopestalotiopsis sonneratae (MFLU 19-0781, holotype). a Habitat. b, c Leaf spots on Sonneronata alba. d, e Culture on PDA (d-above, e-reverse). $\mathrm{f}-\mathrm{h}$ Colony sporulating on PDA. i Conidiogenous cells giving rise to conidia. $\mathrm{j}-\mathrm{p}$ Conidia. Scale bars: $\mathrm{g}=1000 \mu \mathrm{m}, \mathrm{h}=500 \mu \mathrm{m}$, $\mathrm{i}, \mathrm{j}=20 \mu \mathrm{m}, \mathrm{k}-\mathrm{p}=10 \mu \mathrm{m}$. 
Material examined - THAILAND, Kor Chang, Trat Province, leaf spots of Sonneronata alba, 27 April 2017, Norphanphoun Chada KC01-1 (MFLU 19-0781 dried culture, holotype; PDD, isotype); ex-type-living cultures, MFLUCC 17-1744, TNCC. THAILAND, Kor Chang, Trat Province, leaf spots of Sonneronata alba, 27 April 2017, Norphanphoun Chada KC01-2 (MFLU 19-0782 dried culture, paratype); ex-type-living cultures, MFLUCC 17-1745.

Notes - Neopestalotiopsis sonneratae was isolated from a leaf spot of Sonneronata alba in Thailand. In the phylogenetic analyses of combined genes, $N$. sonneratae forms a sister group to $N$. coffea-arabicae (strain HGUP4015) and $N$. thailandica (in this study) (Fig. 1). However, there are significant differences in morphological characteristics; $N$. coffea-arabicae and $N$. thailandica conidia are smaller $(N$. coffea-arabicae: $16-20 \times 5-7 \mu \mathrm{m}, N$. thailandica: $(20-) 21-25(-25.5) \times$ $(5.7-) 6-7(-7.3) \mu \mathrm{m})$ with both species containing longer apical appendages ( $N$. coffea-arabicae: 11-16 $\mu \mathrm{m}, N$. thailandica: (30-)32. 5-38(-40) $\mu \mathrm{m})$ and $N$. thailandica having two apical appendages (Song et al. 2013).

Neopestalotiopsis thailandica Norphanphoun \& K.D. Hyde, sp. nov.

Fig. 7

Index Fungorum number: IF556439; Facesoffungi number: FoF 05775

Etymology - refers to the country where the fungus was collected, Thailand.

Holotype - MFLU 19-0783

Associated with leaf spots of Rhizophora mucronata Lam. Symptoms small irregular to subcircular shape, brown, slightly sunken spots appear on adaxial surface leaves of $R$. mucronata, which later expand outwards on the surface of the leaves (Fig. 7c). Small auburn spots appeared initially and then gradually enlarged, changing to tawny circular ring spots with a dark mahogany border and jagged edge. They were usually $>5$ circulars, which occurred on a single affected leaf. In severe cases, lesions spread evenly on the leaves (Fig. 7b). Asexual morph: Conidiomata pycnidial, globose, brown, semi-immersed on PDA, releasing conidia in a black, slimy, globose, glistening mass. Conidiophores distinct. Conidiogenous cells discrete to lageniform, hyaline, smooth- and thin-walled, proliferating 1-2 times percurrently, collarette present and not flared. Conidia (20-)21-25(-25.5) × 6-7(-7.5) $\mu \mathrm{m}$ (mean $\pm \mathrm{SD}=22.6 \pm 1.3 \times 6.6 \pm 0.5 \mu \mathrm{m})$, fusiform to clavate, straight to slightly curved, 4(-7)-septate; basal cell obconic with a truncate base, hyaline or sometimes pale brown, thin- and smooth-walled, (2.5-)3-4(-4.5) $\mu \mathrm{m}$ long (mean $\pm \mathrm{SD}=3.7 \pm 0.5$ $\mu \mathrm{m})$; threemedian cells (12-)12.5-15(-16) $\mu \mathrm{m}$ long (mean $\pm \mathrm{SD}=14.2 \pm 0.9 \mu \mathrm{m})$, brown, septa and periclinal walls darker than rest of the cell, versicolored, wall rugose; second cell from base pale brown, (4-)4.5-5(-5.5) $\mu \mathrm{m}$ long (mean $\pm \mathrm{SD}=4.6 \pm 0.3 \mu \mathrm{m}$ ); third cell brown, (3.5-)11-26(-27.5) $\mu \mathrm{m}$ long (mean $\pm \mathrm{SD}=4.5 \pm 0.4 \mu \mathrm{m}$ ); fourth cell brown, $(4-) 5-5.5(-6) \mu \mathrm{m}$ long (mean $\pm \mathrm{SD}=5.1$ $\pm 0.6 \mu \mathrm{m}$ ); apical cell $(3.5-) 4-5.5(-6) \mu \mathrm{m}$ long (mean $\pm \mathrm{SD}=4.7 \pm 0.6 \mu \mathrm{m}$ ), hyaline, conic to acute; with 1-2 tubular appendages on apical cell, inserted at different loci but in a crest at the apex of the apical cell, unbranched, flexuous, (30-)32.5-38(-40) $\mu \mathrm{m}$ long (mean $\pm \mathrm{SD}=34.5 \pm 3.7 \mu \mathrm{m}$ ); single basal appendage, tubular, unbranched, centric, $(3-) 6-9(-10) \mu \mathrm{m}$ long (mean $\pm \mathrm{SD}=7.6 \pm$ $2.2 \mu \mathrm{m})$.

Culture characteristics - Colonies on PDA reaching 5-6 $\mathrm{cm}$ diam after $7 \mathrm{~d}$ at room temperature $\left( \pm 25^{\circ} \mathrm{C}\right)$, under light $12 \mathrm{hr} /$ dark $12 \mathrm{hr}$, colonies filamentous to circular, medium dense, aerial mycelium on surface flat or raised, with filiform margin (curled margin), fluffy, white from above and reverse; fruiting bodies black.

Material examined - THAILAND, Kor Chang, Trat Province, leaf spots of Rhizophora mucronata, 27 April 2017, Norphanphoun Chada KC11-1 (MFLU 19-0783 dried culture, holotype; PDD, isotype); ex-type-living cultures, MFLUCC 17-1730, TNCC.THAILAND, Kor Chang, Trat Province, leaf spots of Rhizophora mucronata, 27 April 2017, Norphanphoun Chada KC11-2 (MFLU 19-0784 dried culture, paratype); ex-type-living cultures, MFLUCC 17-1731.

Notes - The collections (MFLUCC 17-1730, MFLUCC 17-1731) were observed and introduced as $N$. thailandica. The new species is introduced with the type from Rhizophora mucronata from Trat Province in Thailand. Neopestalotiopsis thailandica is most similar to 


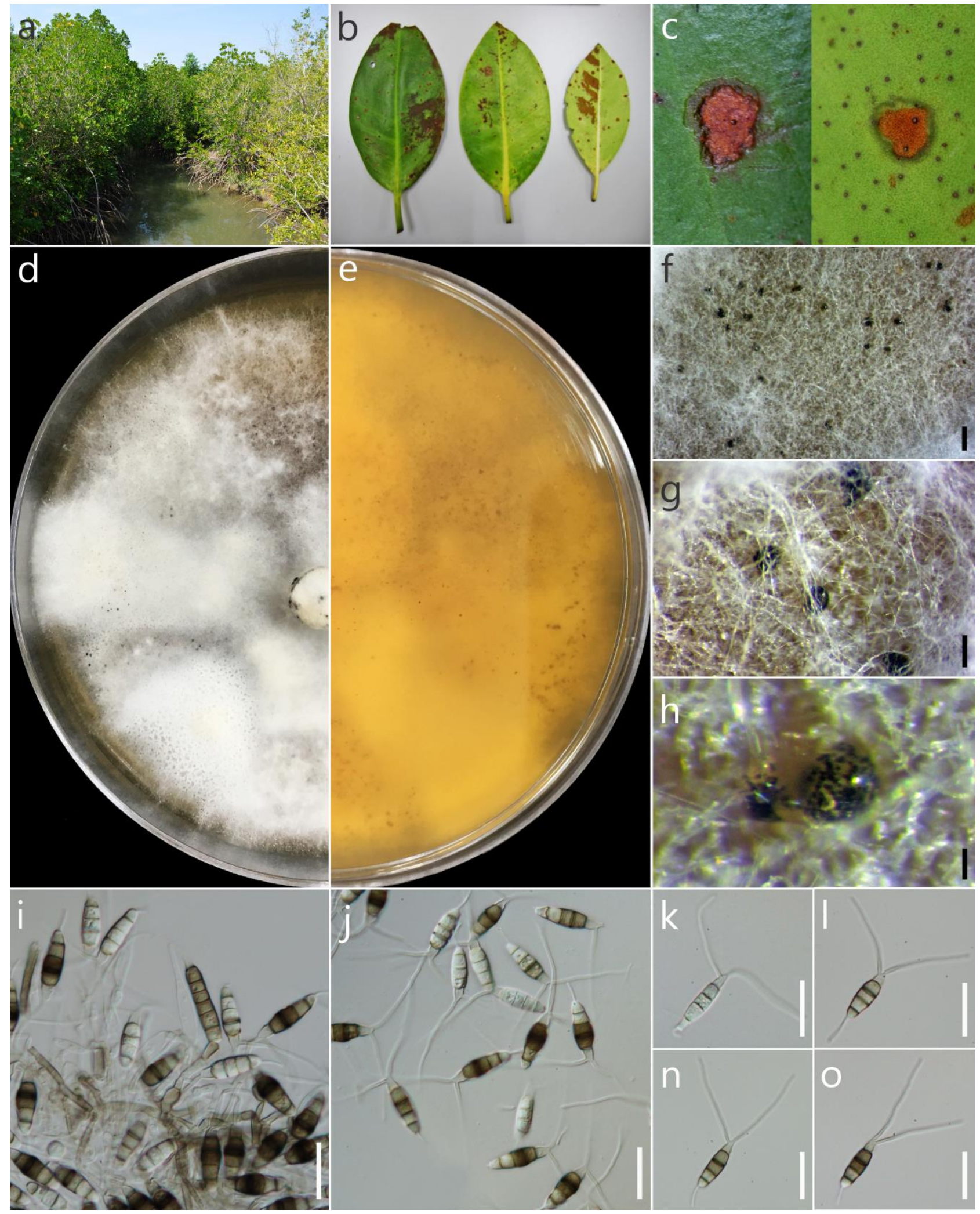

Figure 7 - Neopestalotiopsis thailandica (MFLU 19-0783, holotype). a Habitat. b, c Leaf spots on Rhizophora mucronata. d, e Culture on PDA (d-above, e-reverse). $\mathrm{f}-\mathrm{h}$ Colony sporulating on PDA. $\mathrm{i}$ Conidiogenous cells giving rise to conidia. $\mathrm{j}-\mathrm{o}$ Conidia. Scale bars: $\mathrm{f}=500 \mu \mathrm{m}, \mathrm{g}=200 \mu \mathrm{m}$, $\mathrm{h}=50 \mu \mathrm{m}, \mathrm{i}-\mathrm{O}=20 \mu \mathrm{m}$.

$N$. petila and N. rhizophorae (both species are introduced in this study) with its conidial size. The morphological differences between $N$. petila and $N$. rhizophorae have been mentioned above as notes of $N$. rhizophorae (Table 3 ). However, based on the combined gene phylogenetic analysis, $N$. 
thailandica is separated from other species in the genus and is sister to $N$. coffea-arabicae (HGUP4015, Song et al. 2013), which was isolated from leaves of Coffea arabica L. and $N$. sonneratae (in this study) from leaf spots of Sonneronata alba (Fig. 1). However, their morphology is different. Neopestalotiopsis thailandica differs from $N$. coffea-arabicae by having larger conidia (N. thailandica: $(20-) 21-25(-25.5) \times 6-7(-7.5)$ vs. $N$. coffea-arabicae: $16-20 \times 5-7)$ ), and longer apical appendages (N. thailandica:(30-)32.5-38(-40) vs. N. coffea-arabicae:11-16) (Table 3). The morphological differences between $N$. thailandica and $N$. sonneratae are mentioned above under the notes of $N$. sonneratae. Thus, based on morphology and phylogeny it is considered that $N$. thailandica is a novel species.

The second phylogenetic tree (Pestalotiopsis) comprised 70 taxa with the outgroup, Neopestalotiopsis saprophytica (MFLUCC 12-0282). The total length of the dataset was 1440 characters including alignment gaps, 1-380, 381-538, 539-779, 780-979, 980-1147, and 11481440 corresponding to ITS1+ ITS2, 5.8S, $\beta$-tubulin (exon), $\beta$-tubulin (intron), EF1 $\alpha$ (exon), and EF1 $\alpha$ (intron), respectively. The combined dataset contained 887 constant, 174 parsimony uninformative and 379 parsimony informative characters. The combined dataset was analyzed using MP, ML and BI. The trees generated under different optimality criteria were essentially similar in topology and did not differ significantly (data not shown). The descriptive statistics generated from MP analysis based on the combined dataset of ITS1+ITS2, 5.8S, $\beta$-tubulin (exon), $\beta$-tubulin (intron), $\mathrm{EF} 1 \alpha$ (exon), and $\mathrm{EF} 1 \alpha$ (intron) were $\mathrm{TL}=1664, \mathrm{CI}=0.510, \mathrm{RI}=0.685, \mathrm{RC}=$ $0.349, \mathrm{HI}=0.490$. The best scoring likelihood tree selected with a final value for the combined dataset $=-10362.397645$.

Pestalotiopsis rhizophorae Norphanphoun, T.C. Wen \& K.D. Hyde, sp. nov.

Fig. 9 Index Fungorum number: IF556440; Facesoffungi number: FoF 05781

Etymology - refers to the host from which the fungus was isolated, Rhizophora apiculate Blume.

Holotype - MFLU 19-0785

Associated with leaf spots of Rhizophora apiculata Blume. Symptoms small irregular spots shape, rufous, slightly sunken spots adaxial surface leaves of $R$. apiculata, which later expand outwards on the surface of the leaves (Fig. 9c). Small rufous spots appeared initially and then gradually enlarged, changing to tawny circular ring spots with a dark brown border and smooth edge. They were usually few circulars, which occurred on a single affected leaf (Fig. 9b). Asexual morph: Conidiomata 2 0-7 $0 \mu \mathrm{m}$ diam, pycnidial, globose, brown, semi-immersed on PDA, rreleasing conidia in a black, slimy, globose, glistening mass. Conidiophores indistinct. Conidiogenous cells discrete to lageniform, hyaline, smooth- and thin-walled, 10-20 $01-2 \mu \mathrm{m}$, proliferating 1-2 times percurrently, collarette present and not flared. Conidia (17-)17.5-23(-23.5) $\times(5.5-) 6-6.5(-7) \mu \mathrm{m}$ (mean $\pm \mathrm{SD}=20 \pm 1.6 \times 6.3 \pm 0.5 \mu \mathrm{m}$ ), fusiform to clavate, straight to slightly curved, 4-septate; basal cell obconic with a truncate base, hyaline or sometimes pale brown, thin- and smooth-walled, (2-)3-3.5(-5) $\mu \mathrm{m}$ long (mean $\pm \mathrm{SD}=3.7 \pm 0.7 \mu \mathrm{m}$ ); three median cells (11-)11.5-14(-14.5) $\mu \mathrm{m}$ long (mean $\pm \mathrm{SD}=13.2 \pm 1.2 \mu \mathrm{m})$, brown, septa and periclinal walls darker than rest of the cell, concolorous, wall rugose; second cell from base pale brown, (3.5-)4$5(-5.5) \mu \mathrm{m}$ long (mean $\pm \mathrm{SD}=4.6 \pm 0.6 \mu \mathrm{m})$; third cell brown, $(3-) 4-4.5(-5) \mu \mathrm{m}$ long (mean $\pm \mathrm{SD}$ $=4.1 \pm 0.6 \mu \mathrm{m})$; fourth cell brown, $(3.5-) 4-5(-5.5) \mu \mathrm{m}$ long (mean $\pm \mathrm{SD}=4.6 \pm 0.5 \mu \mathrm{m})$; apical cell $(1.8-) 2-3(-4.5) \mu \mathrm{m}$ long (mean $\pm \mathrm{SD}=3.1 \pm 0.5 \mu \mathrm{m})$, hyaline, conic to acute; with $1-2$ tubular appendages on apical cell, inserted at different loci but in a crest at the apex of the apical cell, unbranched, flexuous, (7.5-)8-13(-14.5) $\mu$ m long (mean $\pm \mathrm{SD}=11.4 \pm 2.4 \mu \mathrm{m}$ ); single basal appendage, tubular, unbranched, centric, $1.5-4.5(-5) \mu \mathrm{m}$ long (mean $\pm \mathrm{SD}=2.5 \pm 1.1 \mu \mathrm{m}$ ).

Culture characteristics - Colonies on PDA reaching 5-6 cm diam after $7 \mathrm{~d}$ at room temperature $\left( \pm 25^{\circ} \mathrm{C}\right)$, under light $12 \mathrm{hr} /$ dark $12 \mathrm{hr}$, colonies filamentous to circular, medium dense, aerial mycelium on surface flat or raised, with filiform margin (curled margin), fluffy, white from above and reverse; fruiting bodies black. 


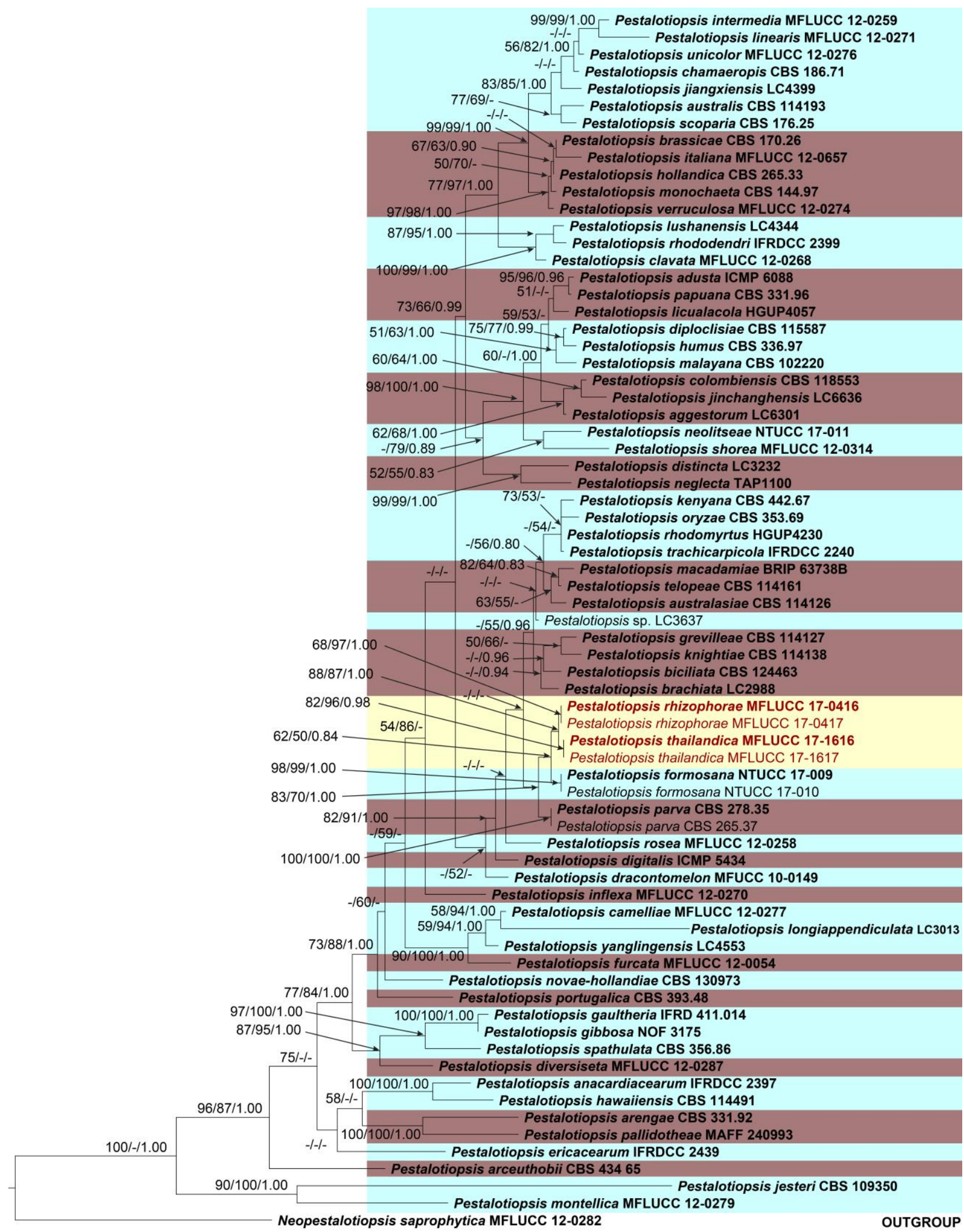

30.0

Figure 8 - One of the 1000 most parsimonious trees obtained from a heuristic search of combined ITS, $\beta$-tubulin and EF1 $\alpha$ sequence data for the genus Pestalotiopsis. The tree is rooted to Neopestalotiopsis saprophytica (MFLUCC 12-0282). Maximum parsimony and maximum likelihood bootstrap values $\leq 50 \%$. Bayesian posterior probabilities $\leq 0.90$ (MPBS/MLBS/PPBY) are given at the nodes. The species obtained in this study are in red font. Ex-type taxa from other studies are in black bold. 


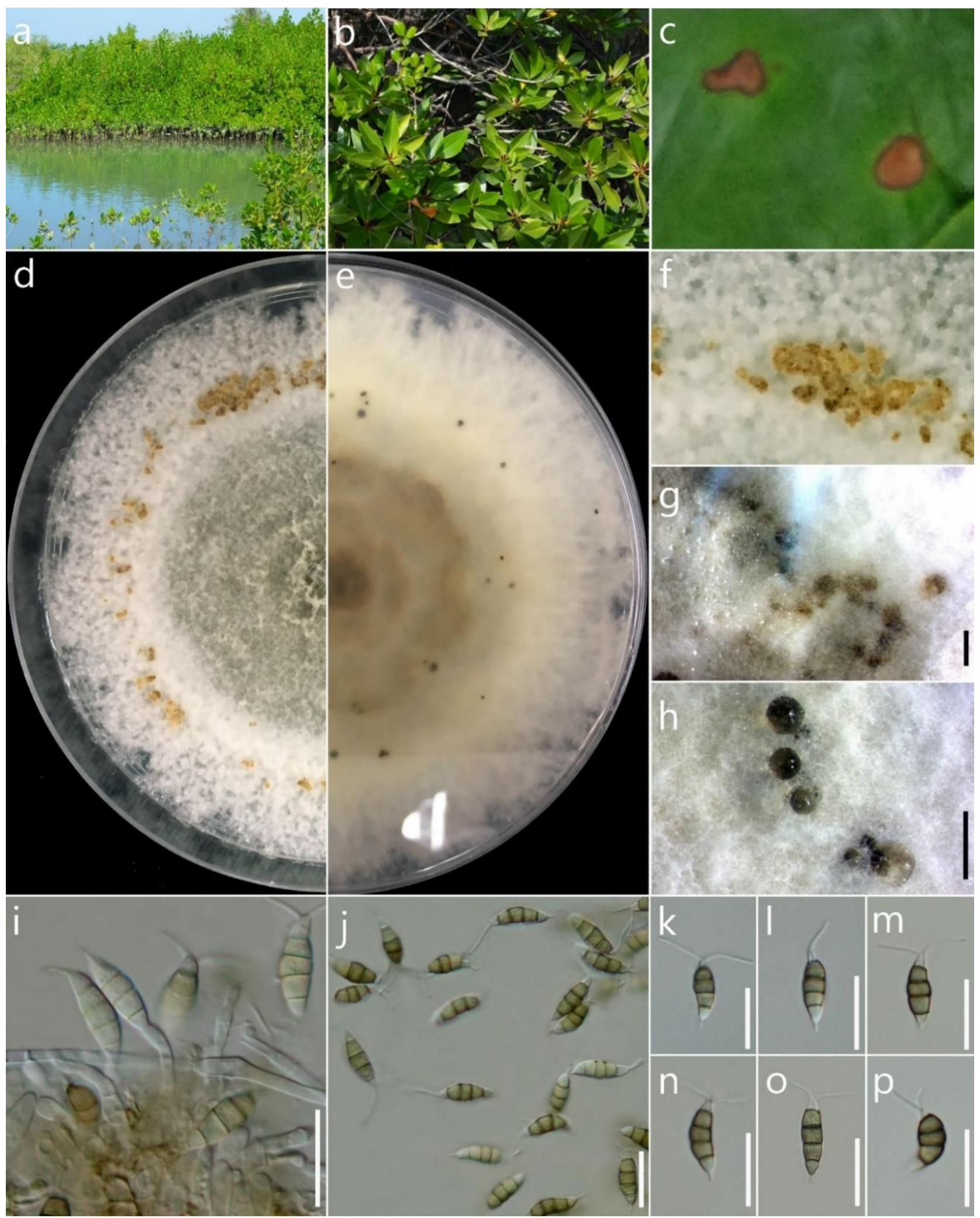

Figure 9 - Pestalotiopsis rhizophorae (MFLU 19-0785, holotype). a Habitat b, c Leaf spots of Rhizophera apiculata. d, e Culture on PDA (d-above, e-reverse). $\mathrm{f}-\mathrm{h}$ Colony sporulating on PDA. $\mathrm{i}$ Conidiogenous cells giving rise to conidia. $\mathrm{j}-\mathrm{p}$ Conidia. Scale bars: $\mathrm{f}-\mathrm{h}=100 \mu \mathrm{m}, \mathrm{i}-\mathrm{p}=20 \mu \mathrm{m}$.

Material examined - THAILAND, The Sirindhorn International Environmental Park, Cha-am District, Phetchaburi Province, leaf spots on Rhizophora apiculata, 30 November 2016, Norphanphoun Chada NNS28a (MFLU 19-0785, holotype; PDD, isotype); ex-type-living cultures, MFLUCC 17-0416, TNCC. THAILAND, The Sirindhorn International Environmental Park, Cha- 
am District, Phetchaburi Province, leaf spots on Rhizophora apiculata, 30 November 2016, Norphanphoun Chada NNS28b (MFLU 19-0786, paratype); living cultures, MFLUCC 17-0417.

Notes - Pestalotiopsis rhizophorae formed a distinct clade in the multi-locus tree and is sister to $P$. formosana (strain NTUCC 17-009), $P$. parva (strain CBS 265.37) and $P$ thailandica (in this study) (Fig. 2). Pestalotiopsis rhizophorae differs from $P$ thailandica and $P$. formosana by shorter conidia (P. rhizophorae: (17-)17.5-23(-23.5) $\mu \mathrm{m}$ vs. $P$ thailandica: (17-)17.5-28(-29) $\mu \mathrm{m}, P$. formosana: $(15-) 18-22(-26) \times(5-) 6-7 \mu \mathrm{m})$; shorter apical appendages $(P$. rhizophorae: $(7.5-) 8-$ 13(-14.5) $\mu \mathrm{m}$ vs. $P$ thailandica: $(5.5-) 11-34(-38) \mu \mathrm{m})$ and basal appendages $(P$. rhizophorae: (1.3-)1.5-4.5(-5) $\mu \mathrm{m}$ vs. $P$ thailandica: $(2-) 2.5-9.5(-10) \mu \mathrm{m}, P$. formosana: $(2-) 3-5(-6) \mu \mathrm{m})$ (Table 4, Ariyawansa and Hyde 2018, Maharachchikumbura et al. 2014). Pestalotiopsis rhizophorae is similar to $P$. parva in conidial size $(P$. parva: $(16-) 16.5-20(-21) \times 5-7(-7.5) \mu \mathrm{m})$ but differs by having 1-2 apical appendages (Table 4, Ariyawansa and Hyde 2018, Maharachchikumbura et al. 2014). The two species (P. rhizophorae, P. thailandica) are also different in four base pairs in ITS, one base pair in $\beta$-tubulin and EF1 $\alpha$. Therefore, the collection in the present study is designated as a new species.

Pestalotiopsis thailandica Norphanphoun, Doilom \& K.D. Hyde, sp. nov.

Fig. 10

Index Fungorum number: IF556441; Facesoffungi number: FoF 05782

Etymology - refers to the country where the fungus was collected, Thailand.

Holotype - MFLU 19-0787

Isolated from asymptomatic leaf of Rhizophora apiculata Blume. Asexual morph: Conidiomata pycnidial, globose, brown, semi-immersed on PDA, releasing conidia in a black, slimy, globose, glistening mass. Conidiophores indistinct. Conidiogenous cells discrete to lageniform, hyaline, smooth- and thin-walled, proliferating 1-2 times percurrently, collarette present and not flared. Conidia (17-)17.5-28(-29) $\times(4.9-) 5.5-6.5(-7.1) \mu \mathrm{m}$ (mean $\pm \mathrm{SD}=23.3 \pm$ $3.0 \times 5.8 \pm 0.5 \mu \mathrm{m}$ ), fusiform to clavate, straight to slightly curved, 4-septate; basal cell obconic with a truncate base, hyaline or sometimes pale brown, thin- and smooth-walled, $(1.8-) 2-4(-6) \mu \mathrm{m}$ long (mean $\pm \mathrm{SD}=3.9 \pm 1.3 \mu \mathrm{m})$; three median cells $(12-) 12.5-16(-18) \mu \mathrm{m}$ long (mean $\pm \mathrm{SD}=$ $15.3 \pm 1.5 \mu \mathrm{m})$, brown, septa and periclinal walls darker than rest of the cell, versicolored, wall rugose; second cell from base pale brown, (4-)4.5-6(-7) $\mu \mathrm{m}$ long (mean $\pm \mathrm{SD}=5.6 \pm 0.8 \mu \mathrm{m}$ ); third cell brown, (3.5-)4-4.5(-5.5) $\mu \mathrm{m}$ long (mean $\pm \mathrm{SD}=4.6 \pm 0.5 \mu \mathrm{m})$; fourth cell brown, (3.5)4-5(-6.5) $\mu \mathrm{m}$ long (mean $\pm \mathrm{SD}=5.1 \pm 0.7 \mu \mathrm{m})$; apical cell $(2-) 3.5-4(-6) \mu \mathrm{m}$ long (mean $\pm \mathrm{SD}=$ $4.2 \pm 1.0 \mu \mathrm{m})$, hyaline, conic to acute; with 1-2 tubular appendages on apical cell, inserted at same loci at the apex of the apical cell, unbranched, flexuous, (5.5-)11-34(-38) $\mu \mathrm{m}$ long (mean $\pm \mathrm{SD}=$ $22.2 \pm 8.5 \mu \mathrm{m})$; single basal appendage, tubular, unbranched, centric, $(2-) 2.5-9.5(-10) \mu \mathrm{m}$ long (mean $\pm \mathrm{SD}=4 \pm 2.0 \mu \mathrm{m}$ ).

Culture characteristics - Colonies on PDA reaching 5-6 $\mathrm{cm}$ diam after $7 \mathrm{~d}$ at room temperature $\left( \pm 25^{\circ} \mathrm{C}\right)$, under light $12 \mathrm{hr} /$ dark $12 \mathrm{hr}$, colonies filamentous to circular, medium dense, aerial mycelium on surface flat or raised, with filiform margin (curled margin), fluffy, white from above and reverse; fruiting bodies black.

Material examined - THAILAND, The Sirindhorn International Environmental Park, Chaam, Cha-am District, Phetchaburi Province, asymptomatic leaf of Rhizophora apiculata, 30 November 2016, Mingkwan Doilom NNSE03AL (MFLU 19-0787, holotype; PDD, isotype); extype-living cultures, MFLUCC 17-1616, TNCC. THAILAND, The Sirindhorn International Environmental Park, Cha-am, Cha-am District, Phetchaburi Province, asymptomatic leaf of Rhizophora apiculata, 30 November 2016, Mingkwan Doilom NNSE03DL (MFLU 19-0788, paratype); living cultures, MFLUCC 17-1617.

Notes - Pestalotiopsis thailandica isolated as an endophyte from a living leaf of Rhizophora apiculata is introduced here as a new species. In the phylogenetic analyses based on combined genes, the species appeared as a distinct species represented by two strains and is sister to $P$. rhizophorae (in this study) (Fig. 2). Although these two species are found on the same host and 


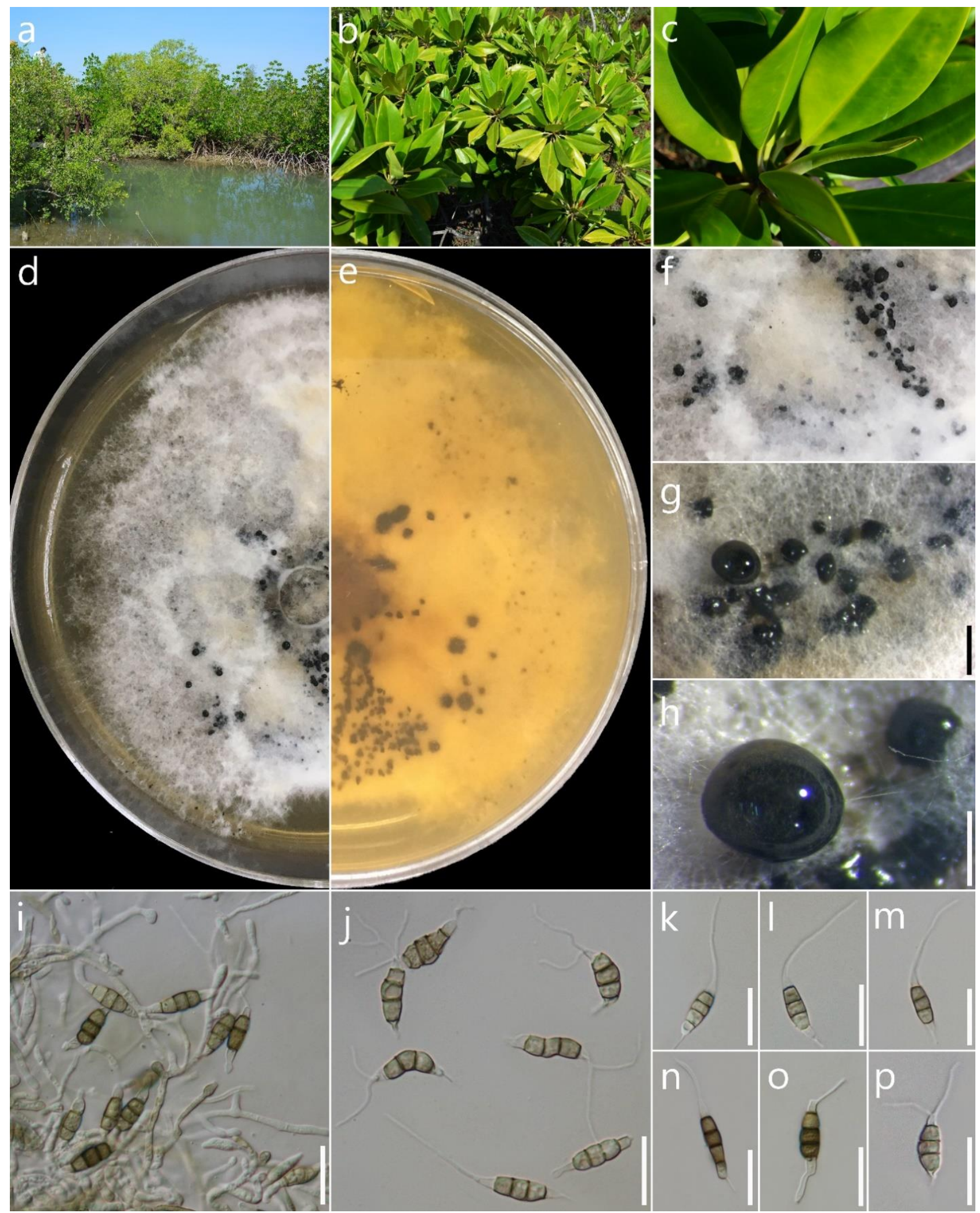

Figure 10 - Pestalotiopsis thailandica (MFLU 19-0787, holotype). a Habitat. b, c Leaf of Rhizophera apiculata. d, e Culture on PDA (d-above, e-reverse). $\mathrm{f}-\mathrm{h}$ Colony sporulating on PDA. $\mathrm{i}$ Conidiogenous cells giving rise to conidia. $\mathrm{j}-\mathrm{p}$ Conidia. Scale bars: $\mathrm{g}=1000 \mu \mathrm{m}, \mathrm{h}=100 \mu \mathrm{m}$, $\mathrm{i}-\mathrm{p}=20 \mu \mathrm{m}$.

location, $P$. thailandica differs by having larger conidia (P. rhizophorae: $(17-) 17.5-23(-23.5) \times$ $(5.5-) 6-6.5(-7) \mu \mathrm{m})$ and longer apical appendages $(P$. rhizophorae: $(7.5-) 8-13(-14.5) \mu \mathrm{m})$ than $P$. rhizophorae (Table 4) (see notes under $P$. rhizophorae). In the phylogenetic analyses, $P$. 
rhizophorae is also related to $P$. formosana (NTUCC 17-009) and $P$. parva (CBS 265. 37). However, Pestalotiopsis formosana and $P$. parva are different from $P$. thailandica by having smaller conidia (P. formosana: (15-)18-22(-26) $\mu \mathrm{m}, P$. parva: $(16-) 16.5-20(-21) \mu \mathrm{m})$ and shorter apical appendages $(P$. formosana: $(8-) 11-16(-20) \mu \mathrm{m}, P$. parva: $(6-) 6.5-12(-13) \mu \mathrm{m})$ with $2-3$ tubular apical appendages (Table 4, Ariyawansa \& Hyde 2018, Maharachchikumbura et al. 2014).

The third phylogenetic tree comprised 27 taxa, with Neopestalotiopsis natalensis (CBS 138. 41) as the outgroup taxon. The total length of the dataset was 1404 characters including alignment gaps, 1-335, 336-493, 494-742, 743-925, 926-1093 and 1094-1404 corresponding to ITS1+ ITS2, 5. 8S, $\beta$-tubulin (exon), $\beta$-tubulin (intron), EF1 $\alpha$ (exon), and EF1 $\alpha$ (intron), respectively). The combined dataset contained 1122 constant, 193 parsimony uninformative and 89 parsimony informative characters. The combined dataset was analyzed using MP, ML and BI. The trees generated under different optimality criteria were essentially similar in topology and did not differ significantly (data not shown). The descriptive statistics generated from MP analysis based on the combined dataset of ITS1+ITS2, 5.8S, $\beta$-tubulin (exon), $\beta$-tubulin (intron), EF1 $\alpha$ (exon), and $\mathrm{EF} 1 \alpha$ (intron) were $\mathrm{TL}=386, \mathrm{CI}=0.832, \mathrm{RI}=0.823, \mathrm{RC}=0.685, \mathrm{HI}=0.168$. The best scoring likelihood tree selected with a final value for the combined dataset $=-3905.071762$.

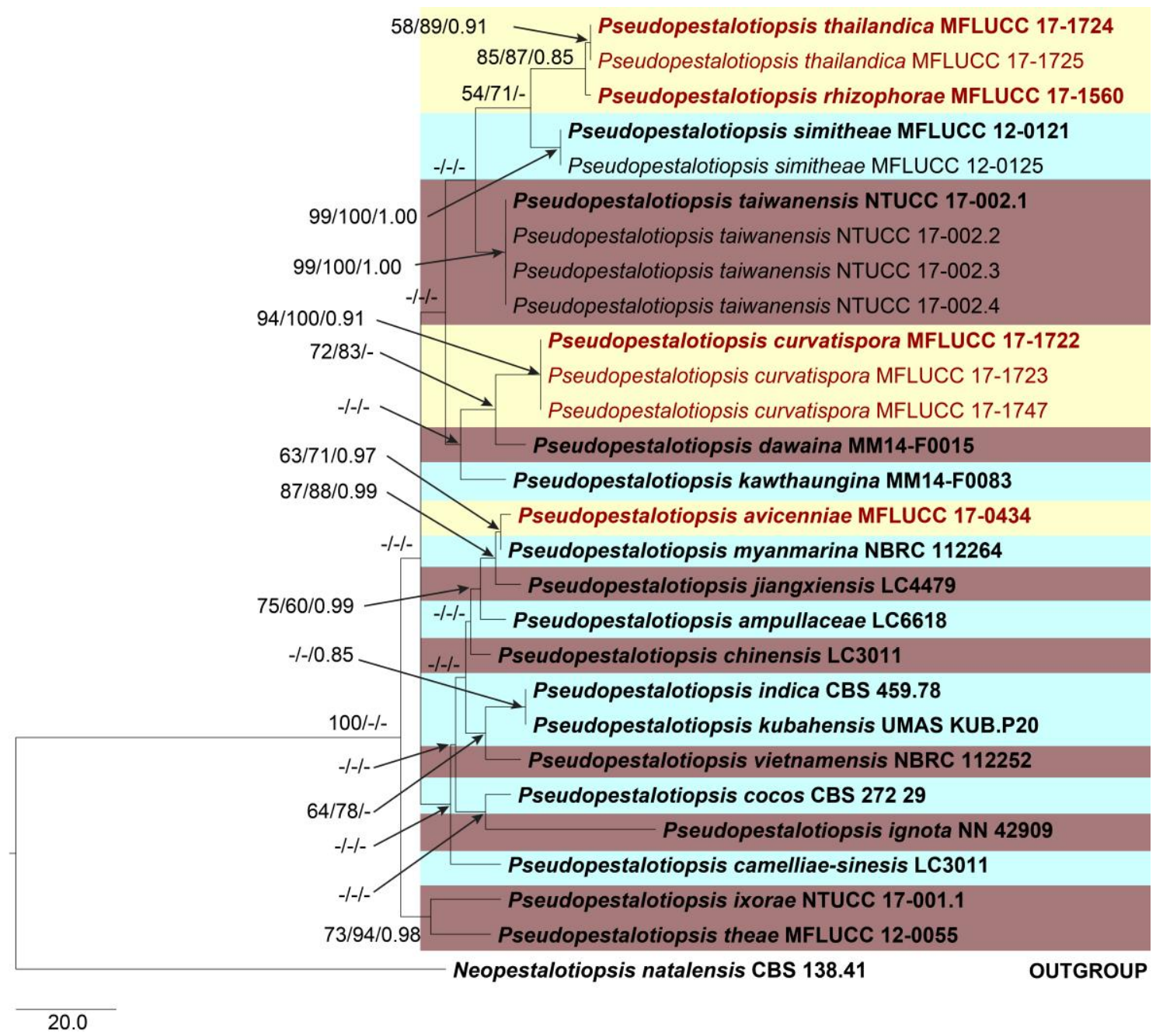

Figure 11 - One of the 93 most parsimonious trees obtained from a heuristic search of combined ITS, $\beta$-tubulin and EF1 $\alpha$ sequence data of the genus Pseudopestalotiopsis. The tree is rooted with Neopestalotiopsis natalensis (CBS 138. 41). Maximum parsimony and maximum likelihood bootstrap values $\leq 50 \%$, Bayesian posterior probabilities $\leq 0.90$ (MPBS/MLBS/PPBY) are given at the nodes. The species obtained in this study are in red font. Ex-type taxa from other studies are in black bold. 
Pseudopestalotiopsis avicenniae Norphanphoun, T.C. Wen \& K.D. Hyde, sp. nov.

Index Fungorum number: IF556442; Facesoffungi number: FoF 05776

Etymology - refers to the host from which the fungus was isolated, Avicennia marina (Forssk.) Vierh.

Holotype - MFLU 19-0789

Associated with leaf spots of Avicennia marina (Forssk.) Vierh. Symptoms circular to irregular, grayish-brown, slightly sunken spots adaxial surface leaves of A. marina, which later expand outwards on the surface of the leaves (Fig. 12c). Small black spots appeared initially and then gradually enlarged, changing to beige circular ring spots with a black border and smooth edge. They were usually $>5$ circulars, which occurred on a single affected leaf. In severe cases, lesions spread evenly on the leaves (Fig. 12b). Asexual morph: Conidiomata 300-1000 $\mu \mathrm{m}$ diam, pycnidial, globose, brown, semi-immersed on PDA, releasing conidia in a black, slimy, globose, glistening mass. Conidiophores indistinct. Conidiogenous cells discrete to lageniform, hyaline, smooth- and thin-walled, 3-6 $\times 2-56 \mu \mathrm{m}$, proliferating 1-2 times percurrently, collarette present and not flared. Conidia (22-)22.5-26.5(-27) $\times(5-) 5.5-6(-6.5) \mu \mathrm{m}$ (mean $\pm \mathrm{SD}=23.9 \pm 1.4 \times 6 \pm$ $0.4 \mu \mathrm{m})$, fusiform to clavate, straight to slightly curved, 4-septate; basal cell obconic with a truncate base, hyaline or sometimes pale brown, thin- and smooth-walled, (3-)4-4.5(-6) $\mu \mathrm{m}$ long (mean \pm $\mathrm{SD}=4 \pm 0.7 \mu \mathrm{m})$; three median cells $(15-) 15.5-17(-18) \mu \mathrm{m}$ long (mean $\pm \mathrm{SD}=16.3 \pm 0.8 \mu \mathrm{m})$, brown, septa and periclinal walls darker than rest of the cell, versicolored, wall rugose; second cell from base pale brown, (4.5-)5-6(-6.5) $\mu \mathrm{m}$ long (mean $\pm \mathrm{SD}=5.5 \pm 0.5 \mu \mathrm{m})$; third cell brown, $(4.5-) 5-5.5(-6) \mu \mathrm{m}$ long (mean $\pm \mathrm{SD}=4.9 \pm 0.4 \mu \mathrm{m}$ ); fourth cell brown, $(5-) 5.5-6(-7) \mu \mathrm{m}$ long (mean $\pm \mathrm{SD}=5.9 \pm 0.5 \mu \mathrm{m})$; apical cell $(2-) 2.5-4(-5) \mu \mathrm{m}$ long (mean $\pm \mathrm{SD}=3.5 \pm 1.0 \mu \mathrm{m})$, hyaline, conic to acute; with 1-3 tubular appendages on apical cell, inserted at different loci but in a crest at the apex of the apical cell, unbranched, flexuous, (14-)15.5-28.5(-35.5) $\mu \mathrm{m}$ long (mean \pm $\mathrm{SD}=21.3 \pm 5.9 \mu \mathrm{m})$; single basal appendage, tubular, unbranched, centric, $(2-) 3-4(-4.5) \mu \mathrm{m}$ long (mean $\pm \mathrm{SD}=3.1 \pm 0.7 \mu \mathrm{m}$ ).

Culture characteristics - Colonies on PDA reaching 5-7 cm diam after $7 \mathrm{~d}$ at room temperature $\left( \pm 25^{\circ} \mathrm{C}\right)$, under light $12 \mathrm{hr} /$ dark $12 \mathrm{hr}$, colonies filamentous to circular, medium dense, aerial mycelium on surface flat or raised, with filiform margin (curled margin), fluffy, white from above and reverse; fruiting bodies black.

Material examined - THAILAND, The Sirindhorn International Environmental Park, Chaam, Cha-am District, Phetchaburi Province, leaf spots of Avicennia marina, 30 November 2016, Norphanphoun Chada NNS05-1 (MFLU 19-0789, holotype; PDD, isotype); ex-type-living cultures, MFLUCC 17-0434, TNCC.

Notes - The combined phylogenetic tree showed that Ps. avicenniae is sister to Ps. jiangxiensis (strain LC4479) and Ps. elaeidis (=Ps. myanmarina, strain NBRC 112264) (Fig. 1, Liu et al. 2017, Nozawa et al. 2017). Pseudopestalotiopsis avicenniae is morphologically similar to Ps. jiangxiensis, but phylogenetically clearly distinct as an independent lineage (Fig. 3). Pseudopestalotiopsis avicenniae is phylogenetically closer to Ps. elaeidis (strain NBRC 112264), which was collected from Averrhoa carambola in Myanmar, but can be distinguished by its larger conidia (Ps. elaeidis: 31-38.5 × 6.5-9 $\mu \mathrm{m}$ ) and 2-3 apical appendages (Table 5, Nozava et al. 2017).

Pseudopestalotiopsis curvatispora Norphanphoun, T.C. Wen \& K.D. Hyde, sp. nov.

Fig. 13

Index Fungorum number: IF556443; Facesoffungi number: FoF 05777

Etymology - in reference to the Latin word "curvatus" in reference to the shape of the conidia.

Holotype - MFLU 19-0790

Associated with leaf spots of Rhizophora mucronata Blume. Symptoms small subcircular, grayish brown, slightly sunken spots adaxial surface leaves of $R$. mucronata, which later expand outwards on the surface of the leaves (Fig. 13c). Small brown spots appeared initially and then 


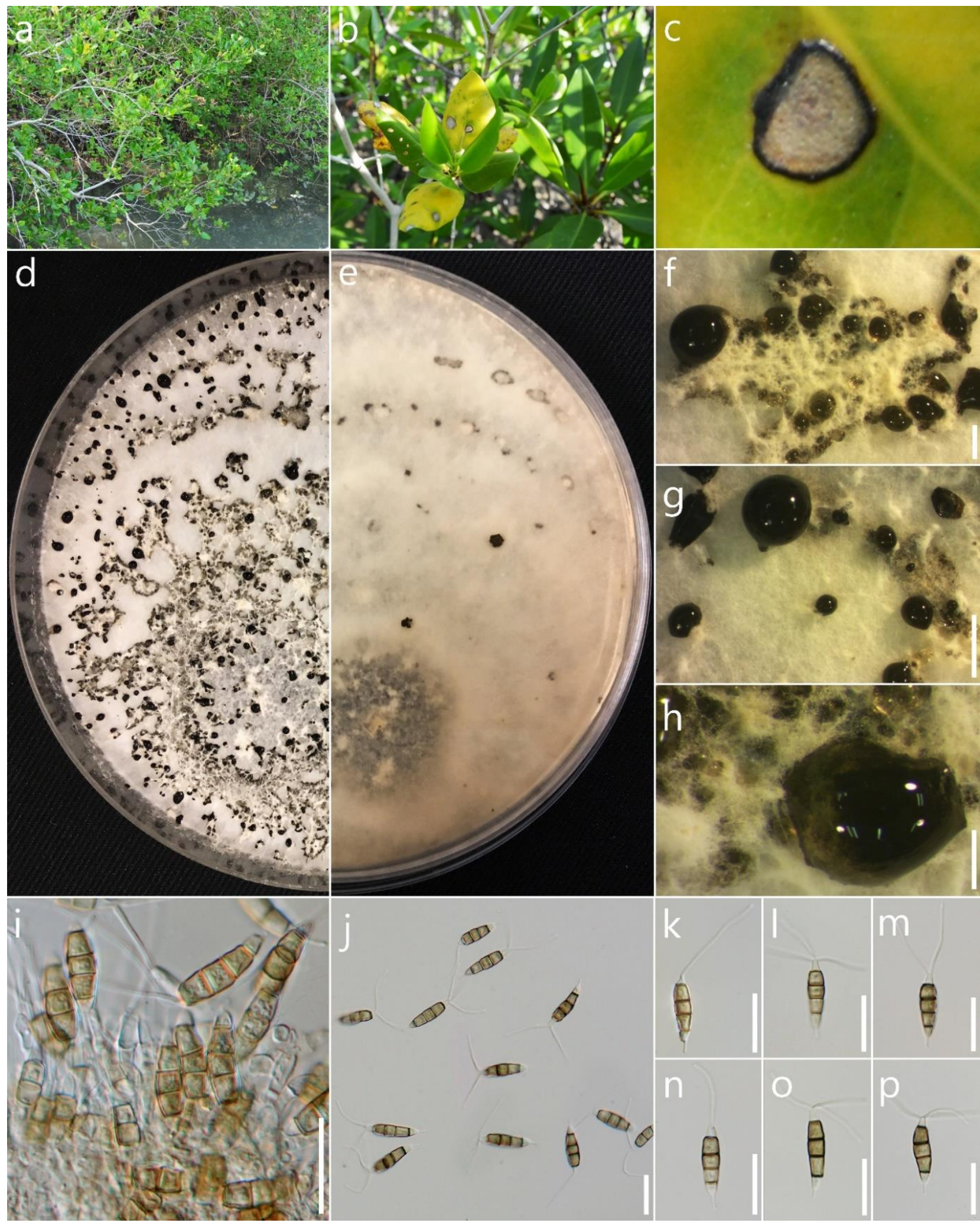

Figure 12 - Pseudopestalotiopsis avicenniae (MFLU 19-0789, holotype). a Habitat. b, c Leaf spots on Avicennia marina. $\mathrm{d}$, e Culture on PDA (d-above, e-reverse). $\mathrm{f}-\mathrm{h}$ Colony sporulating on PDA. $\mathrm{i}$ Conidiogenous cells giving rise to conidia. $\mathrm{j}-\mathrm{p}$ Conidia. Scale bars: $\mathrm{f}=1000 \mu \mathrm{m}, \mathrm{g}=200 \mu \mathrm{m}$, $\mathrm{h}=500 \mu \mathrm{m}, \mathrm{i}-\mathrm{p}=20 \mu \mathrm{m}$.

gradually enlarged, changing to beige circular ring spots with a dark brown border and smooth edge. They were usually $>5$ circulars, which occurred on a single affected leaf. In severe cases, lesions spread evenly on the leaves (Fig. 13b). Asexual morph: Conidiomata 200-400 $\mu \mathrm{m}$ diam, 
pycnidial, globose, brown, semi-immersed on PDA, rreleasing conidia in a black, slimy, globose, glistening mass. Conidiophores indistinct. Conidiogenous cells discrete to lageniform, hyaline, smooth- and thin-walled, 5.5-11 $\times 3-5.5 \mu \mathrm{m}$, proliferating 1-2 times percurrently, collarette present and not flared. Conidia (18.5-)22-25(-26.5) $\times(6-) 6.5-7 \mu \mathrm{m}$ (mean $\pm \mathrm{SD}=23.5 \pm 2 \times 6.6$ $\pm 0.4 \mu \mathrm{m})$, fusiform to clavate, straight to slightly curved, 4-septate; basal cell obconic with a truncate base, hyaline or sometimes pale brown, thin- and smooth-walled, (3-)3.5-4(-5) $\mu \mathrm{m}$ long (mean $\pm \mathrm{SD}=4.3 \pm 0.7 \mu \mathrm{m}$ ); three median cells $(11-) 13-14(-15) \mu \mathrm{m}$ long (mean $\pm \mathrm{SD}=13.8 \pm 1.1$ $\mu \mathrm{m})$, brown, septa and periclinal walls darker than rest of the cell, versicolored, wall rugose; second cell from base pale brown, 4-5 $\mu \mathrm{m}$ long (mean $\pm \mathrm{SD}=4.5 \pm 0.5 \mu \mathrm{m}$ ); third cell brown, (3.5-)4-5(5.5) $\mu \mathrm{m}$ long (mean $\pm \mathrm{SD}=4.4 \pm 0.6 \mu \mathrm{m}$ ); fourth cell brown, $(3.5-) 4-5(-5.5) \mu \mathrm{m}$ long (mean $\pm \mathrm{SD}$ $=4.6 \pm 0.6 \mu \mathrm{m})$; apical cell $(3.5-) 4-6(-7) \mu \mathrm{m}$ long (mean $\pm \mathrm{SD}=5.1 \pm 1.1 \mu \mathrm{m})$, hyaline, conic to acute; with 1-2 tubular appendages on an apical cell, inserted at different loci but in a crest at the apex of the apical cell, unbranched or branched irregularly along their length resulting in 1-2 branches, flexuous, (10-)20-29(-35) $\mu \mathrm{m}$ long (mean $\pm \mathrm{SD}=22.7 \pm 8.1 \mu \mathrm{m}$ ); single basal appendage, tubular, unbranched, centric, (5.5-)9-12(-13.5) $\mu \mathrm{m}$ long (mean $\pm \mathrm{SD}=9.2 \pm 2 \mu \mathrm{m}$ ).

Culture characteristics - Colonies on PDA reaching 3-4 cm diam after $7 \mathrm{~d}$ at room temperature $\left( \pm 25^{\circ} \mathrm{C}\right)$, under light $12 \mathrm{hr} /$ dark $12 \mathrm{hr}$, colonies filamentous to circular, medium dense, aerial mycelium on surface flat or raised, with filiform margin (curled margin), fluffy, white from above and reverse; fruiting bodies black.

Material examined - THAILAND, Trat Province, leaf spots of Rhizophora mucronata, 27 April 2017, Norphanphoun Chada KC12-1, (MFLU 19-0791, holotype; PDD, isotype); ex-typeliving cultures, MFLUCC 17-1722, TNCC. THAILAND, Trat Province, leaf spots of Rhizophora mucronata, 27 April 2017, Norphanphoun Chada KC12-2, (MFL 19-0792, paratype); living cultures, MFLUCC 17-1723. THAILAND, Trat Province, leaf spots of Rhizophora apiculata, 27 April 2017, Norphanphoun Chada KC18-2 (MFLU 19-0790, paratype); ex-type-living cultures, MFLUCC 17-1747.

Notes - The new species Pseudopestalotiopsis curvatispora is introduced, which was isolated from a leaf spot on Rhizophora mucronata, with the morphology of curved conidia and flexuous branched apical appendages. Based on combined gene phylogenetic analyses, it showed that Ps. curvatispora is nested in between Ps. simitheae (MFLUCC 12-0121) and Ps. thailandica (in this study), which are morphologically different (Table 5). Pseudopestalotiopsis simitheae is (MFLUCC 12-0121), distinct from other species by forming a well-separated clade (Fig. 3). This is also supported by morphological differences (larger conidia $(22-30 \mu \mathrm{m})$ and 2-4 tubular, shorter apical and basal appendages (apical appendages: 14.5-26.5 $\mu \mathrm{m}$, basal appendages: 4-6.5 $\mu \mathrm{m}$, Song et al. 2014)). Pseudopestalotiopsis thailandica is different from Ps. simitheae by having larger conidia $((24-) 24.5-30(-30.5) \mu \mathrm{m})$, longer apical appendages $((26.5-) 28-36(-39.5) \mu \mathrm{m})$ and shorter basal appendages $((3.5-) 4.5-5(-6.5) \mu \mathrm{m})$ (Table 5, Song et al. 2014).

Pseudopestalotiopsis rhizophorae Norphanphoun, T.C. Wen \& K.D. Hyde, sp. nov.

Fig. 14 Index Fungorum number: IF556444; Facesoffungi number: FoF 05778

Etymology - refers to the host from which the fungus was isolated, Rhizophora apiculata Blume.

Holotype - MFLU 19-0793

Associated with leaf spots of Rhizophora apiculata Blume. Symptoms irregular shape, pale brown, slightly sunken spots adaxial surface leaves of $R$. apiculata, which later expand outwards on the surface of the leaves (Fig. 14c). Small brown spots appeared initially and then gradually enlarged, changing to pale brown irregular spots with auburn border. They were usually $>5$ circular spots occurred on a single affected leaf. In severe cases, lesions spread evenly on the leaves with defected leaves (Fig. 14b). Asexual morph: Conidiomata 100-200 $\mu \mathrm{m}$ diam, pycnidial, globose, brown, semi-immersed on PDA, releasing conidia in a black, slimy, globose, glistening mass. Conidiophores indistinct. Conidiogenous cells discrete to lageniform, hyaline, smooth- and 


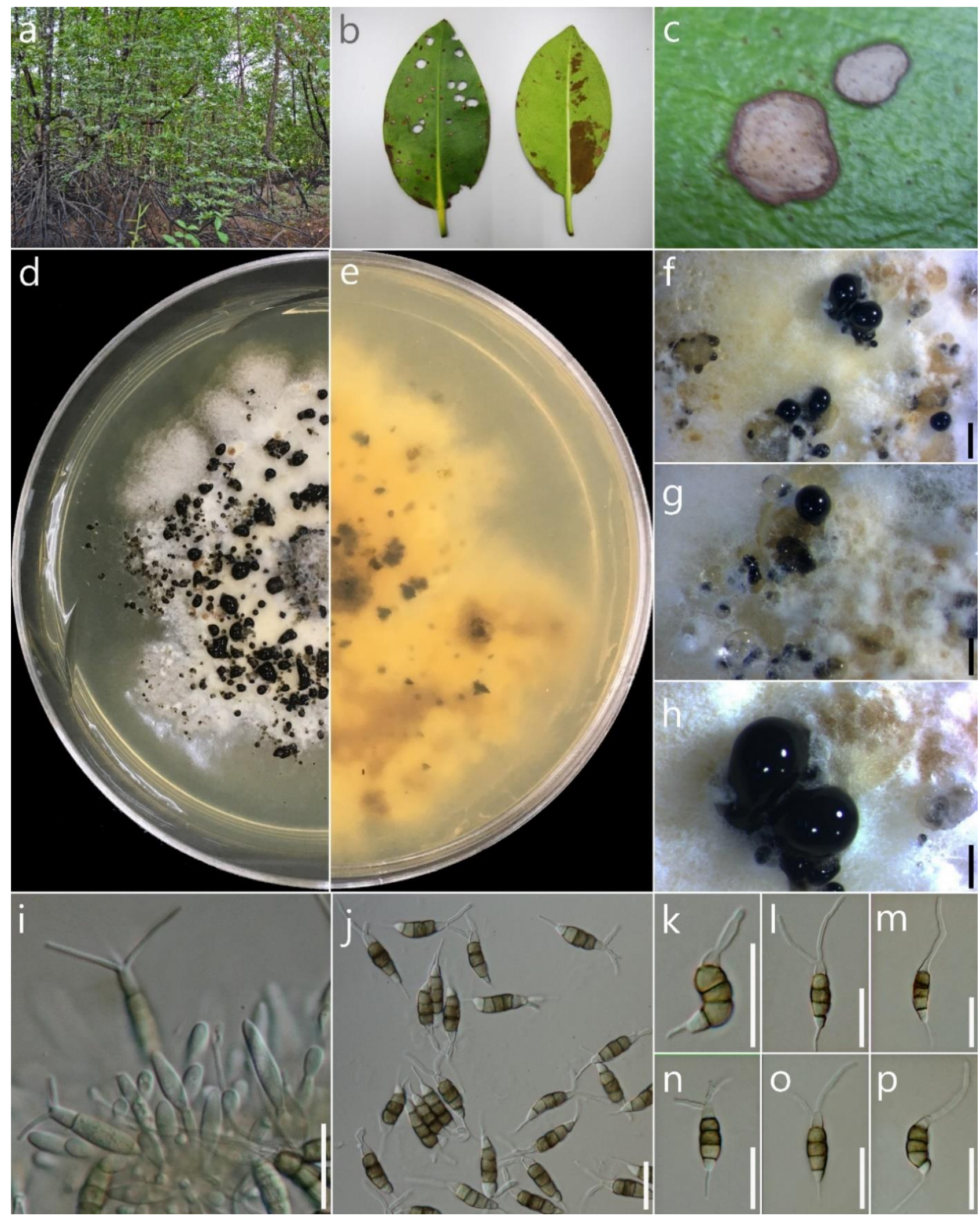

Figure 13 - Pseudopestalotiopsis curvatispora (MFLU 19-0 791, holotype). a Habitat. b, c Leaf spots of Rhizophera mucronata. d, e Culture on PDA (d-above, e-reverse). $\mathrm{f}-\mathrm{h}$ Colony sporulating on PDA. i Conidiogenous cells giving rise to conidia. $\mathrm{j}-\mathrm{p}$ Conidia. Scale bars: $\mathrm{f}=1000 \mu \mathrm{m}$, $\mathrm{g}, \mathrm{h}=500 \mu \mathrm{m}, \mathrm{i}-\mathrm{p}=20 \mu \mathrm{m}$.

thin-walled, 5-8 $\times 5-6 \mu \mathrm{m}$, proliferating 1-2 times percurrently, collarette present and not flared. Conidia (23-)24-29.5(-30) $\times 5-5.5(-6) \mu \mathrm{m}$ (mean $\pm \mathrm{SD}=26 \pm 2.5 \times 5.4 \pm 0.4 \mu \mathrm{m}$ ), fusiform to clavate, straight to slightly curved, 4-septate; basal cell obconic with a truncate base, hyaline or 
sometimes pale brown, thin- and smooth-walled, $(3-) 4-5(-6) \mu \mathrm{m}$ long (mean $\pm \mathrm{SD}=4.5 \pm 0.9$ $\mu \mathrm{m})$; three median cells (14.5-)15-19(-19.5) $\mu \mathrm{m}$ long (mean $\pm \mathrm{SD}=16.4 \pm 2 \mu \mathrm{m}$ ), brown, septa and periclinal walls darker than rest of the cell, versicolored, wall rugose; second cell from base pale brown, (4-)5-5.5(-7) $\mu \mathrm{m}$ long (mean $\pm \mathrm{SD}=5.8 \pm 1 \mu \mathrm{m}$ ); third cell brown, 4-5(-5.5) $\mu \mathrm{m}$ long (mean $\pm \mathrm{SD}=4.7 \pm 0.4 \mu \mathrm{m}$ ); fourth cell brown, $(4.5-) 5.5-6(-7.5) \mu \mathrm{m}$ long (mean $\pm \mathrm{SD}=5.9 \pm 1.0$ $\mu \mathrm{m})$; apical cell (3.5-)4-5(-7) $\mu \mathrm{m}$ long (mean $\pm \mathrm{SD}=5.2 \pm 0.8 \mu \mathrm{m})$, hyaline, conic to acute; with 2-3 tubular appendages on apical cell, inserted at different loci but in a crest at the apex of the apical cell, unbranched, flexuous, (16.5-)18-25(-26) $\mu \mathrm{m}$ long (mean $\pm \mathrm{SD}=22 \pm 3.1 \mu \mathrm{m}$ ); single basal appendage, tubular, unbranched, centric, (3-)3.5-6(-7) $\mu \mathrm{m}$ long (mean $\pm \mathrm{SD}=5 \pm 1.2 \mu \mathrm{m}$ ).

Culture characteristics - Colonies on PDA reaching 5-6 cm diam after $7 \mathrm{~d}$ at room temperature $\left( \pm 25^{\circ} \mathrm{C}\right)$, under light $12 \mathrm{hr} /$ dark $12 \mathrm{hr}$, colonies filamentous to circular, medium dense, aerial mycelium on surface flat or raised, with filiform margin (curled margin), fluffy, white from above and reverse; fruiting bodies black.

Material examined - THAILAND, Ngao, Ranong Province, Ngao Mangrove Forest Research Centre, leaf spots of Rhizophora apiculata, 6 December 2016, Norphanphoun Chada NG38a (MFLU 19-0793, holotype; PDD, isotype); ex-type-living cultures, MFLUCC 17-1560, TNCC.

Notes - Pseudopestalotiopsis rhizophorae formed an independent branch in the phylogeny presented here (Fig. 3) and is closely related to Ps. dawaina (MM14-F0015) and Ps. kubahensisa (UMAS KUB-P20). Pseudopestalotiopsis rhizophorae differs from Ps. dawaina and Ps. kubahensisa in length of appendage in both ends: shorter apical appendage (Ps. dawaina: 20.5-33.5 $\mu \mathrm{m}$, Ps. kubahensisa: $16-29.5 \mu \mathrm{m}$ ) and longer basal appendage (Ps. dawaina: 2.5-6.5 $\mu \mathrm{m}$, Ps. kubahensisa: 3-6 $\mu \mathrm{m}$ ) (Table 5). Thus, Ps. rhizophorae is introduced as a new species.

Pseudopestalotiopsis thailandica Norphanphoun \& K.D. Hyde, sp. nov.

Fig. 15

Index Fungorum number: IF556445; Facesoffungi number: FoF 05779

Etymology - refers to the country where the fungus was collected, Thailand.

Holotype - MFLU 19-0794

Associated with leaf spots of Rhizophora mucronata Blume. Symptoms subcircular to the irregular shape, pale brown, slightly sunken spots adaxial surface leaves of $R$. mucronata, which later expand outwards on the surface of the leaves (Fig. 15c). Small auburn spots appeared initially and then gradually enlarged, changing to pale-auburn circular ring spots with a dark auburn border. They were usually $>5$ circulars, which occurred on a single affected leaf. In severe cases, lesions spread evenly on the leaves (Fig. 15b). Asexual morph: Conidiomata 250-500 $\mu \mathrm{m}$ diam, pycnidial, globose, brown, semi-immersed on PDA, releasing conidia in a black, slimy, globose, glistening mass. Conidiophores indistinct. Conidiogenous cells discrete to lageniform, hyaline, smooth- and thin-walled, proliferating 1-2 times percurrently, collarette present and not flared. Conidia (2424.5-30(-30.5) $\times(5-) 5.5-6(-6.7) \mu \mathrm{m}($ mean $\pm \mathrm{SD}=26.6 \pm 2.2 \times 5.9 \pm 0.3 \mu \mathrm{m})$, fusiform to clavate, straight to slightly curved, 4-septate; basal cell obconic with a truncate base, hyaline or sometimes pale brown, thin- and smooth-walled, (3.5-)4-5(-6.6) $\mu \mathrm{m}$ long (mean $\pm \mathrm{SD}=4.4 \pm 1 \mu \mathrm{m}$ ); three median cells (13.5-) 16-18(-19) $\mu \mathrm{m}$ long (mean $\pm \mathrm{SD}=17.2 \pm 1.5 \mu \mathrm{m})$, brown, septa and periclinal walls darker than rest of the cell, versicolored, wall rugose; second cell from base pale brown, (5)5.5-6(-7.5) $\mu \mathrm{m}$ long (mean $\pm \mathrm{SD}=5.8 \pm 0.9 \mu \mathrm{m})$; third cell brown, (5-)5.5-6(-6.2) $\mu \mathrm{m}$ long (mean $\pm \mathrm{SD}=5.6 \pm 0.5 \mu \mathrm{m}$ ); fourth cell brown, (5.5-)6-6.5(-7) $\mu \mathrm{m}$ long (mean $\pm \mathrm{SD}=6.2 \pm 0.6$ $\mu \mathrm{m})$; apical cell (3.5-)4.5-5(-7) $\mu \mathrm{m}$ long (mean $\pm \mathrm{SD}=4.5 \pm 1.1 \mu \mathrm{m}$ ), hyaline, conic to acute; with 1-2 tubular appendages on apical cell, inserted at different loci but in a crest at the apex of the apical cell, unbranched, flexuous, $(26.5-) 28-36(-39.5) \mu \mathrm{m}$ long (mean $\pm \mathrm{SD}=31.3 \pm 3.9 \mu \mathrm{m}$ ); single basal appendage, tubular, unbranched, centric, (3.5-)4.5-5(-6.5) $\mu \mathrm{m}$ long (mean $\pm \mathrm{SD}=4.8$ $\pm 1 \mu \mathrm{m})$.

Culture characteristics - Colonies on PDA reaching 5-6 cm diam after $7 \mathrm{~d}$ at room temperature $\left( \pm 25^{\circ} \mathrm{C}\right)$, under light $12 \mathrm{hr} /$ dark $12 \mathrm{hr}$, colonies filamentous to circular, medium dense, aerial mycelium on surface flat or raised, with filiform margin (curled margin), fluffy, white from above and reverse; fruiting bodies black. 


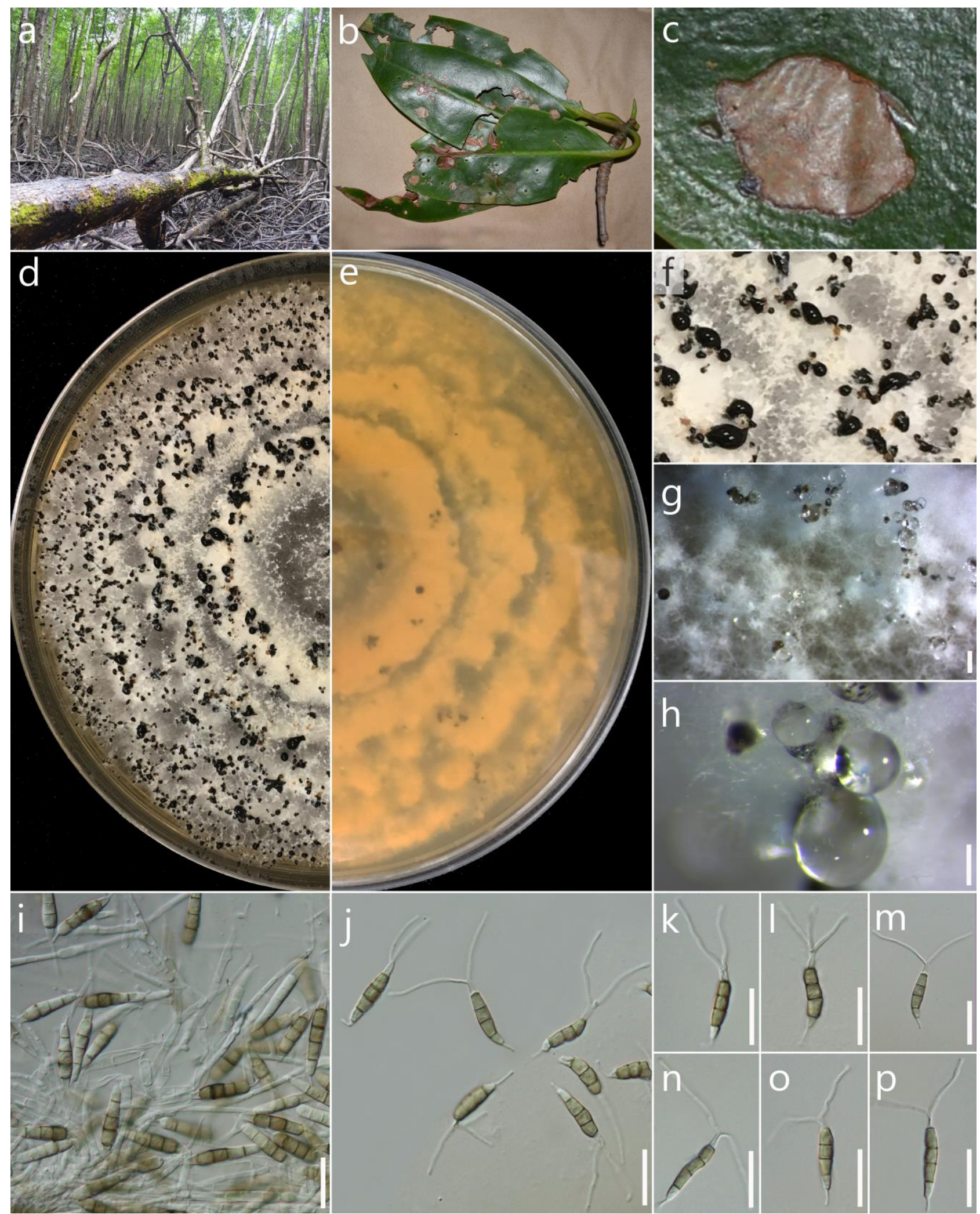

Figure 14 - Pseudopestalotiopsis rhizophorae (MFLU 19-0793, holotype). a Habitat. b, c Leaf spots of Rhizophora apiculata. d, e Culture on PDA (d-above, e-reverse). $\mathrm{f}-\mathrm{h}$ Colony sporulating on PDA. i Conidiogenous cells giving rise to conidia. j-p Conidia. Scale bars: $\mathrm{g}=500 \mu \mathrm{m}, \mathrm{h}=200$ $\mu \mathrm{m}, \mathrm{i}-\mathrm{p}=20 \mu \mathrm{m}$.

Material examined - THAILAND, Chanthaburi Province, leaf spots of Rhizophora mucronata, 27 April 2017, Norphanphoun Chada KC21-1 (MFLU 19-0794, holotype; PDD, isotype); ex-type-living cultures, MFLUCC 17-1724, TNCC. THAILAND, Chanthaburi Province, 
leaf spots of Rhizophora mucronata, 27 April 2017, Norphanphoun Chada KC21-12 (MFLU 190795, paratype); ex-type-living cultures, MFLUCC 17-1725.

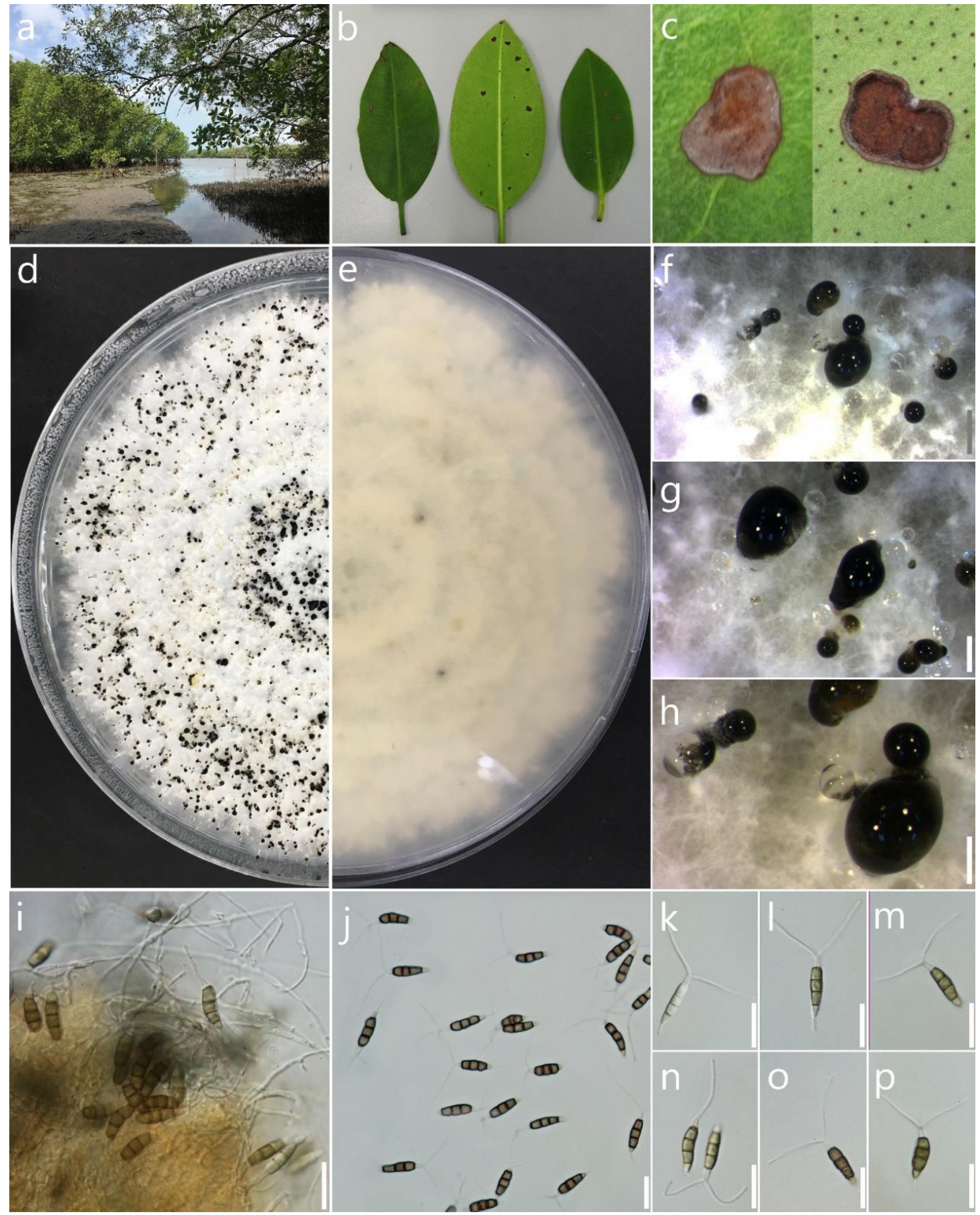

Figure 15 - Pseudopestalotiopsis thailandica (MFLU 19-0794, holotype). a Habitat. b, c Leaf spots on Rhizophora mucronata. d, e Culture on PDA (d-above, e-reverse). f-h Colony sporulating on PDA. i Conidiogenous cells giving rise to conidia. $j-p$ Conidia. Scale bars: $f=1000 \mu \mathrm{m}$, $\mathrm{g}, \mathrm{h}=500 \mu \mathrm{m}, \mathrm{i}-\mathrm{p}=20 \mu \mathrm{m}$. 
Notes - The new species, Pseudopestalotiopsis thailandica was isolated from a leaf spot from Rhizophora mucronata from Chanthaburi Province, Thailand. Based on the combined gene phylogenetic analysis, it showed that Ps. thailandica is sister to Ps. curvatispora (in this study), and morphology differences as mentioned in the notes of Ps. curvatispora. Thus, it is considered that Ps. thailandica is a novel species.

\section{Discussion}

Mangrove forests occurring at the interface of terrestrial and marine ecosystems harbour a rich biological diversity of plants, animals and microorganisms (Beger et al. 2010). However, the phylogenetic and functional description of microbial diversity in mangrove ecosystems has not been addressed to the same extent as for other environments. A previous study on mangrove fungi reported some pathogenic fungi causing leaf spots on Rhizophora, e.g. leaf spots on Rhizophora mangle in Puerto Rico caused by Anthostomella (Stevens 1920), leaf spots on R. mangle in Florida caused by Cercospora (McMillan 1964), leaf spots on Rhizophora sp. caused by Pestalotiopsis sp., Colletotrichum sp. and Polystigma sonneratiae (Hyde \& Cannon 1992, Xu et al. 2009). Heart and butt rot disease on Rhizophora sp. caused by Heterobasidion annosum, Ganoderma, Phellinus sp., and Inonotus sp. (Ryvarden 2000, Fox 2001, Sakayarojet al. 2012) and canker and dieback on Rhizophora sp. caused by Phytophthora cinnamomi (Vollbrecht et al. 1995, Barnard 2000, Kinge \& Mih 2011) have also been reported. A leaf spot of Avicennia marina caused by Pseudocercospora avicenniae (Shivas et al. 2009) was recorded in Queensland, Australia. Thatoi et al. (2013) reported more than 200 species of endophytic fungi from mangroves in India. Norphanphoun et al. (2018) reported three novel species of Cytospora (C. lumnitzericola, $C$. thailandica and $C$. xylocarpi) from Lumnitzera racemosa, Xylocarpus granatum and $X$. moluccensis from Phetchaburi and Ranong provinces in Thailand, and Kumar et al. (2019) reported an endophytic species, Neopestalotiopsis alpapicalis on $R$. apiculata and $R$. mucronata from Krabi Province, Thailand.

In this study, we examined 22 strains isolated from leaf collections from Phetchaburi, Ranong and Trat Provinces in Thailand. As a result of these sampling endeavors, we described 12 novel species, including six novel species of Neopestalotiopsis (N. acrostichi, N. brachiata, N. petila, N. rhizophorae, $N$. sonneratae, $N$. thailandica), two novel species of Pestalotiopsis (P. rhizophorae, $P$. thailandica) and four novel species of Pseudopestalotiopsis (Ps. avicenniae, Ps. curvatispora, $P s$. rhizophorae and Ps. thailandica), based on morphological and molecular phylogenetic evidence, which clearly differentiated these new species from known species. As many pestalotioid species have overlapping morphological traits, sequence data is essential to resolve these three genera and introduce new species. Combined gene sequences of ITS, $\beta$-tubulin and EF1 $\alpha$ can provide a better resolution for Pestalotiopsis and Pesudopestalotiopsis. However, more genes are needed to provide better resolution and support in Neopestalotiopsis. Furthermore, this is the first report of Neopestalotiopsis, Pestalotiopsis and Pseudopestalotiopsis fungi associated on mangrove plants in Thailand (Acrostichum aureum, Avicennia marina, Rhizophora mucronata, $R$. apiculata and Sonneronata alba), which indicates that there may be a high undescribed diversity of fungi in this ecosystem as has been shown elsewhere in terrestrial habitats in northern Thailand (Hyde et al. 2018). We found 12 new species from mangrove ecosystems, however further study is needed to establish whether these species are unique to such hosts and habitats. 
Table 3 Comparison of conidia of Neopestalotiopsis species related to this study.

\begin{tabular}{|c|c|c|c|c|c|}
\hline \multirow{2}{*}{ Species } & \multirow{2}{*}{ Strain } & \multirow{2}{*}{ Conidial size $(\mu \mathrm{m})$} & \multicolumn{2}{|c|}{ Apical appendages } & \multirow{2}{*}{$\begin{array}{c}\text { Basal appendage } \\
\text { Length }(\mu \mathrm{m})\end{array}$} \\
\hline & & & Number & Length $(\mu \mathrm{m})$ & \\
\hline N. acrostichi & $\begin{array}{l}\text { MFLUCC 17-1754/ } \\
\text { MFLUCC 17-1755 }\end{array}$ & $(22-) 23-26(-27.2) \times(5-) 5.5-6.5(-7.1)$ & $3-4(4)$ & $(16-) 19-65(-69.5)$ & $(7-) 12.5-36(-37)$ \\
\hline N. alpapicalis $^{\mathrm{g}}$ & MFLUCC 17-2544 & $14-22.3 \times 5-6.8$ & $1-4(1,2)$ & $5.6-15$ & $3.1-6.4$ \\
\hline N. aotearoa $^{\mathrm{d}}$ & CBS 367.54 & $(19.5-) 21-28(-29) \times(6-) 6.5-8.5(-9)$ & $2-3$ & $(3-) 5-12(-13)$ & $1.5-4$ \\
\hline N. coffea-arabicae $\mathrm{f}^{\mathrm{f}}$ & HGUP4015 & $16-20 \times 5-7$ & $2-4$ & $11-16$ & $3-5$ \\
\hline N. musae & MFLUCC 15-0776 & $18.6-2594.1-5$ & $2-3$ & $16.3-25$ & $4.6-10.3$ \\
\hline N. picean $a^{\mathrm{d}}$ & CBS 394.48 & $(19-) 19.5-25(-26) \times(7-) 7.5-9(-9.5)$ & 3 & $(19-) 21-31(-33)$ & $6-23$ \\
\hline N. protearum ${ }^{\mathrm{a}}$ & CBS 114178 & $(14-) 16-17(-18) \times(6.5-) 8-9(-10)$ & $2-4$ & $(10-) 15-17(-22)$ & $(2-) 3-3.5(-5)$ \\
\hline N. brachiata & MFLUCC $17-1555$ & $(18-) 18.5-25(-26) \times(4.7-) 5.5-6(-6.3)$ & $1-3$ & $(8.5-) 9.5-33(-34)$ & $(3.5-) 4-9(-10)$ \\
\hline N. rhizophorae & $\begin{array}{l}\text { MFLUCC 17-1550/ } \\
\text { MFLUCC 17-1551 }\end{array}$ & $(20-) 20.5-27(-27.5) \times(6-) 6.5-7.5(-8.2)$ & $1-4(3)$ & $(6-) 12.5-22(-24)$ & $(2.5-) 4-9.5(-10)$ \\
\hline N. rosicola ${ }^{\mathrm{c}}$ & CFCC 51992 & $(18.9-) 20.2-25.5(-26.2) \times(5-) 5.5-8(-8.5)$ & $2-4(2,3)$ & $(16.5-) 17-22.8(-25.9)$ & $2-9.5$ \\
\hline N. samarangensis ${ }^{\mathrm{e}}$ & MFLUCC 12-0233 & $18-21 \times 6.5-7.5$ & 3 & $12-18$ & $3.5-5.2$ \\
\hline N. sonneratae & $\begin{array}{l}\text { MFLUCC 17-1744/ } \\
\text { MFLUCC 17-1745 }\end{array}$ & $(21.6-) 24-26(-28.2) \times(6.8-) 7-7.5(-8.1)$ & $1-3$ & $(5.3-) 7-8(-13.8)$ & $(2.5-) 3-4(-4.7)$ \\
\hline N. surinamensis ${ }^{\mathrm{d}}$ & CBS 450.74 & $(23-) 24-28(-29) \times(7-) 7.5-9(-9.5)$ & $2-3$ & $(15-) 18-27(-28)$ & Up to $5-7$ \\
\hline N. thailandica & $\begin{array}{l}\text { MFLUCC 17-1730/ } \\
\text { MFLUCC 17-1731 }\end{array}$ & $(20-) 21-25(-25.5) \times(5.7-) 6-7(-7.3)$ & $1-2$ & $(30-) 32.5-38(-40)$ & $(3-) 6-9(-10)$ \\
\hline N. petila & $\begin{array}{l}\text { MFLUCC 17-1737/ } \\
\text { MFLUCC 17-1738 }\end{array}$ & $(20-) 21-26.5(-27.5) \times(5.6-) 6-7(-7.8)$ & $2-3$ & $(21-) 22-29(-33)$ & $(2-) 3-8(-9)$ \\
\hline
\end{tabular}

Strains in this study are in bold.

${ }^{\mathrm{a}}$ Crous et al. (2011); ${ }^{\mathrm{b}} \mathrm{Hyde}$ et al. (2016); ${ }^{\mathrm{c} J i a n g}$ et al. (2018); ${ }^{\mathrm{d}}$ Maharachchikumbura et al. (2014); ${ }^{\mathrm{e}}$ Maharachchikumbura et al. (2013); ${ }^{\mathrm{f}}$ Song et al. (2013); ${ }^{\mathrm{g}}$ Kumar et al. (2019).

Table 4 Comparison of conidia of Pestalotiopsis species related to this study.

\begin{tabular}{|c|c|c|c|c|c|}
\hline \multirow{2}{*}{ Species } & \multirow{2}{*}{ Strain } & \multirow{2}{*}{ Conidial size $(\mu \mathrm{m})$} & \multicolumn{2}{|c|}{ Apical appendages $(\mu \mathrm{m})$} & \multirow{2}{*}{ Basal appendage $(\mu \mathrm{m})$} \\
\hline & & & Number & Length & \\
\hline P. formosana ${ }^{\text {a }}$ & NTUCC 17-009 & $(15-) 18-22(-26) \times(5-) 6-7$ & $2-3$ & $(8-) 11-16(-20)$ & $(2-) 3-5(-6)$ \\
\hline P. parva ${ }^{\mathrm{b}}$ & CBS 265.37 & $(16-) 16.5-20(-21) \times 5-7(-7.5)$ & $2-3$ & $(6-) 6.5-12(-13)$ & $2-4$ \\
\hline P. rhizophorae & $\begin{array}{l}\text { MFLUCC 17-0416/ } \\
\text { MFLUCC 17-0417 }\end{array}$ & $(17-) 17.5-23(-23.5) \times(5.5-) 6-6.5(-7)$ & $1-2$ & $(7.5-) 8-13(-14.5)$ & $(1.3-) 1.5-4.5(-5)$ \\
\hline P.thailandica & $\begin{array}{l}\text { MFLUCC 17-1616/ MFLUCC } \\
17-1617\end{array}$ & $(17-) 17.5-28(-29) \times(4.9-) 5.5-6.5(-7.1)$ & $1-2$ & $(5.5-) 11-34(-38)$ & $(2-) 2.5-9.5(-10)$ \\
\hline
\end{tabular}

Strains in this study are in bold.

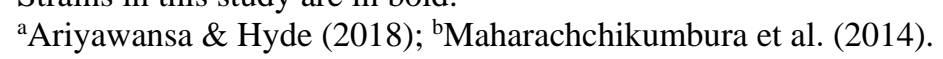


Table 5 Comparison of conidia of Pseudopestalotiopsis species related to this study.

\begin{tabular}{|c|c|c|c|c|c|}
\hline \multirow{2}{*}{ Species } & \multirow{2}{*}{ Strain } & \multirow{2}{*}{ Conidial size $(\mu \mathrm{m})$} & \multicolumn{2}{|c|}{ Apical appendages $(\mu \mathrm{m})$} & \multirow{2}{*}{$\begin{array}{c}\text { Basal appendage } \\
(\mu \mathrm{m})\end{array}$} \\
\hline & & & Number & Length & \\
\hline Ps. ampullacea ${ }^{\mathrm{b}}$ & LC6618 & $21-31.5 \times 6.5-9$ & $2-3$ & $17-25$ & $3.5-7$ \\
\hline Ps.avicenniae & MFLUCC 17-0434 & $(22-) 22.5-26.5(-27) \times(5-) 5.5-6(-6.4)$ & $1-3$ & $(14-) 15.5-28.5(-35.5)$ & $(2-) 3-4(-4.5)$ \\
\hline Ps. chinensis ${ }^{\mathrm{b}}$ & LC3011 & $25.5-35.5 \times 6-9$ & $2-3$ & $24-41$ & $5-12$ \\
\hline Ps.rhizophorae & MFLUCC 17-1560 & $(18.5-) 22-25(-26.5) \times(6.2-) 6.5-7(-7.2)$ & $1-2$ & $(10.2-) 20-29(-35)$ & $(5.5-) 9-12(-13.5)$ \\
\hline Ps. dawaina ${ }^{\mathrm{d}}$ & MM14-F0015 & $22-31 \times 8-9.5$ & 3 & $20.5-33.5$ & $2.5-6.5$ \\
\hline $\begin{array}{l}\text { Ps. elaeidis } \\
(=\text { Ps. myanmarina })^{\mathrm{c}}\end{array}$ & NBRC 112264 & $31-38.5 \times 6.5-9$ & $2-3$ & $22.5-38.5$ & unbranched \\
\hline Ps. jiangxiensis ${ }^{\mathrm{b}}$ & LC4479 & $22-29 \times 6-9$ & $2-4(3)$ & $16.5-32$ & $6.5-19.5$ \\
\hline Ps. kawthaungina ${ }^{\mathrm{d}}$ & MM14-F0083 & $29.5-34.5 \times 7-9$ & 3 & $28-41$ & $4.5-9$ \\
\hline Ps. kubahensis ${ }^{\mathrm{a}}$ & UMAS KUB-P20 & $(26-) 27-30(-33) \times 5.6-7.3$ & $2-4(3)$ & $15.9-29.4$ & $3.1-6.0$ \\
\hline Ps.curvatispora & $\begin{array}{l}\text { MFLUCC 17-1722/ } \\
\text { MFLUCC 17-1723 }\end{array}$ & $(18.6-) 19-26(-26.4) \times(5.5-) 6-7(-7.4)$ & $2-3$ & $(5.5-) 6-24(-26.6)$ & $(5.8-) 5-11(-12.2)$ \\
\hline Ps. simitheae $e^{\mathrm{e}}$ & MFLUCC 12-0121 & $22-30 \times 5-6.5$ & $2-4$ & $14.5-26.5$ & $4-6.5$ \\
\hline Ps. taiwanensis ${ }^{\mathrm{f}}$ & NTUCC 17-002.1 & $21-26 \times 6-7$ & $2-5$ & $16-25$ & $3-7$ \\
\hline Ps. thailandica & $\begin{array}{l}\text { MFLUCC 17-1724/ } \\
\text { MFLUCC 17-1725 }\end{array}$ & $(24-) 24.5-30(-30.5) \times(5-) 5.5-6(-6.7)$ & $1-3$ & $(26.5-) 28-36(-39.5)$ & $(3.5-) 4.5-5(-6.6)$ \\
\hline
\end{tabular}

Strain in this study are in bold.

${ }^{\mathrm{a}}$ Lateef et al. (2015); ${ }^{\mathrm{b}}$ Liu et al. (2017); ${ }^{\mathrm{c}}$ Nozawa et al. (2017); ${ }^{\mathrm{d}}$ Nozawa et al. (2018); ${ }^{\mathrm{e}}$ Song et al. 2014; ${ }^{\mathrm{f}}$ Tsai et al. (2018).

\section{Acknowledgements}

This work was supported by the Science and Technology Foundation of Guizhou Province (No. (2017)5788); the Mushroom Research Foundation (MRF), Chiang Rai, Thailand; the Thailand Research Fund and Mae Fah Luang University entitled "Biodiversity, Phylogeny and role of fungal endophytes on above parts of Rhizophora apiculata and Nypa fruticans" (grant no. RSA5980068) and Mae Fah Luang University for a grant "Diseases of mangrove trees and maintenance of good forestry practice" (Grant number: 60201000201) for support. We would like to thank the Ranong Mangrove Forest Research Center, Ranong and the Sirindhorn International Environmental Park, Cha-am, Cha-am District, Phetchaburi, Thailand for allowing us to collect the samples. We are also grateful to Mingkwan Doilom for help us to on species Pestalotiopsis thailandica and Sajeewa S.N. Maharachchikumbura for the comments and suggestion given on this paper to make it a success, Shaun Pennycook, for checking the name of the novel species and Kunhtida Phutthacharoen, Naruemon Huanraluek are thanked for the help given to this study in observing the morphology. 


\section{References}

Abraham S, Basukriadi A, Pawiroharsono S, Sjamsuridzal W. 2015 - Insecticidal activity of ethyl acetate extracts from culture filtrates of mangrove fungal endophytes. Mycobiology 43, 137149.

Alias SA, Jones EBG. 2009 - Marine fungi from mangroves of Malaysia. Institute Ocean and Earth Sciences, University Malaya, 108.

Alias SA, Zainuddin N, Jones EBG. 2010 - Biodiversity of marine fungi in Malaysian mangroves. Botanica Marina 53, 545-554.

Ariyawansa HA, Hyde KD. 2018 - Additions to Pestalotiopsis in Taiwan. Mycosphere 9, 9991013.

Barnard EL. 2000 - Inonotus root and butt rot of pines in Florida. Plant Pathology Circular 403.

Barr ME. 1975 - The genus Ostreichnion. Mycotaxon 3, 81-88.

Beger M, Grantham HS, Pressey RL, Wilson KA et al. 2010 - Conservationplanning for connectivity across marine, freshwater, andterrestrial realms. Biological Conservation 143, $565-575$.

Bengtsson-Palme J, Ryberg M, Hartmann M, Branco S, et al. 2013 - Improved software detection and extraction of ITS 1 and ITS2 from ribosomal ITS sequences of fungi and other eukaryotes for analysis of environmental sequencing data. Methods in Ecology and Evolution 4, 914919.

Carbone I, Kohn LM. 1999 - A method for designing primer sets for speciation studies in filamentous ascomycetes. Mycologia 91, 553-556.

Castresana J. 2000 -Selection of conserved blocks from multiple alignments for their use in phylogenetic analysis. Molecular Biology and Evolution 17, 540-552.

Chaeprasert S, Piapukiew J, Whalley AJ, Sihanonth P. 2010 - Endophytic fungi from mangrove plant species of Thailand: their antimicrobial and anticancer potentials. Botanica Marina 53, $555-564$.

Cribb AB, Cribb JW. 1955 - Marine fungi from Queensland-I. Papers of the University of Queensland. Department of Botany 3, 78-81.

Crous PW, Summerell BA, Swart L. 2011 - Fungal pathogens of Proteaceae. Persoonia 27, 20-45.

Devadatha B, Sarma VV, Jeewon R, Wanasinghe DN et al. 2018 - Thyridariella, a novel marine fungal genus from India: morphological characterization and phylogeny inferred from multigene DNA sequence analyses. Mycological Progress, 1-14.

Doilom M, Manawasinghe IS, Jeewon R, Jayawardena RS et al. 2017 - Can ITS sequence data identify fungal endophytes from cultures? A case study from Rhizophora apiculata. Mycosphere 8, 1869-1892.

Fisher P, Spalding M. 1993 - Protected areas with mangrove habitat. World Conservation Centre, Cambridge, UK.

Fox RTV. 2001 - 'Fungal foes in your garden'. Butt Rot Mycologist 15, 184-185.

Glass NL, Donaldson GC. 1995 - Development of primer sets designed for use with the PCR to amplify conserved genes from filamentous ascomycetes. Applied and Environmental Microbiology 61, 1323-30.

Guba EF. 1961 - Monograph of Pestalotia and Monochaetia. Harvard University Press, Cambridge.

Hall TA. 1999 - BioEdit: a user-friendly biological sequence alignment editor and analysis program for Windows 95/98/NT. Nucleic Acids Symposium Series 41, 95-98.

Hassan MK, Jintana V, Kuittinen S, Pappinen A. 2018 - "Management Practices and Aboveground Biomass Production Patterns of Rhizophora apiculata Plantation: Study from a Mangrove Area in Samut Songkram Province, Thailand". BioResources 13, 7826-7850.

Hu HL, Jeewon R, Zhou DQ, Zhou TX, Hyde KD. 2007 - Phylogenetic diversity of endophytic Pestalotiopsis species in Pinus armandii and Ribes spp.: evidence from rDNA and betatubulin gene phylogenies. Fungal Diversity 24, 1-22. 
Hyde KD, Cannon PF. 1992 - 'Polystigma sonneratiae causing leaf spots on the mangrove genus Sonneratia'. Australian Systematic Botany 5, 415-420.

Hyde KD, Jones EBG. 1988 - Marine mangrove fungi. Marine Ecology 9, 15-33.

Hyde KD, Lee SY. 1995 - Ecology of mangrove fungi and their role in nutrient cycling: What gaps occur in our knowledge?. Hydrobiologia 295, 107-118.

Hyde KD, Jones EBG, Leano E, Pointing SB et al. 1998 - Role of fungi in marine ecosystems. Biodiversity and Conservation 7, 1147-1161.

Hyde KD, Hongsanan S, Jeewon R, Bhat DJ et al. 2016 - Fungal diversity notes 367-490: taxonomic and phylogenetic contributions to fungal taxa. Fungal Diversity 80, 1-270.

Hyde KD, Norphanphoun C, Chen J, Dissanayake AJ et al. 2018 - Thailand's amazing diversity up to $96 \%$ of fungi in northern Thailand are novel. Fungal Diversity 93, 215-239.

Index Fungorum. 2019 - www.indexfungorum.org

Jayasiri SC, Hyde KD, Ariyawansa HA, Bhat J et al. 2015 - The Faces of Fungi database: fungal names linked with morphology, phylogeny and human impacts. Fungal Diversity 74, 3-18.

Jeewon R, Liew ECY, Hyde KD. 2002 - Phylogenetic relationships of Pestalotiopsis and allied genera inferred from ribosomal DNA sequences and morphological characters. Molecular Phylogenetics and Evolution 25, 378-392.

Jeewon R, Liew ECY, Simpson JA, Hodgkiss IJ, Hyde KD. 2003 - Phylogenetic significance of morphological characters in the taxonomy of Pestalotiopsis species. Molecular Phylogenetics and Evolution 27, 372-383.

Jiang N, Bonthond G, Fan XL, Tian CM. 2018 - Neopestalotiopsis rosicola sp. nov. causing stem canker of Rosa chinensis in China. Mycotaxon 133, 271-283.

Jones EBG, Zuccaro A, Mitchell J, Nakagiri A et al. 2009 - 'Phylogenetic position of freshwater and marine Sigmoidea species: introducing a marine hyphomycete Halosigmoidea gen. nov.(Halosphaeriales)'. Botanica Marina 52, 349-359.

Jones EBG and Pang KL. 2012 - 'Tropical aquatic fungi'. Biodiversity and conservation 21, $2403-$ 2423.

Jones G, Ka-Lai Pang KL, Abdel-Wahab MA, Scholz B et al. 2019 - An online resource for marine fungi. Fungal Diversity, https://doi.org/10.1007/s13225-019-00426-5(0123456789().,volV)(0123456789,-().volV) ().

Katoh K, Standley DM. 2013 - MAFFT multiple sequence alignment Software Version 7: improvements in performance and usability. Molecular Biology and Evolution 30, 772-780.

Kinge TR and Mih AM. 2011 - 'Ganoderma ryvardense sp. nov. associated with basal stem rot (BSR) disease of oil palm in Cameroon'. Mycosphere 2, 179-188.

Kohlmeyer J, Kohlmeyer E. 1979 - Marine mycology. The higher fungi. Academic Press, New York, USA, 1-690.

Kumar S, Stecher G, Tamura K. 2015 - MEGA7: Molecular Evolutionary Genetics Analysis version 7.0. Molecular Biology and Evolution 33, 1870-1874.

Kumar V, Cheewangkoon R, Gentekaki E, Maharachchikumbura S et al. 2019 - Neopestalotiopsis alpapicalis sp. nov. a new endophyte from tropical mangrove trees in Krabi Province (Thailand). Phytotaxa 393, 251-262.

Larkin MA, Blackshields G, Brown NP, Chenna R et al. 2007 - Clustal W and Clustal X version 2.0. Bioinformatics 23, 2947-2948.

Lateef AA, Sepiah M, Bolhassan MH. 2015 - Description of Pseudopestalotiopsiskubahensis sp. nov., a new species of microfungi from Kubah National Park, Sarawak, Malaysia. Current Research in Environmental \& Applied Mycology 5, 376-381.

Li J, Jeewon R, Phookamsak R, Bhat DJ et al. 2018 - Marinophialophora garethjonesii gen. et sp. nov.: a new hyphomycete associated with Halocyphina from marine habitats in Thailand. Phytotaxa 345, 1-12.

Liu F, Hou LW, Raza M, Cai L. 2017 - Pestalotiopsis and allied genera from Camellia, with description of 11 new species from China. Scientific Reports 7, 1-19. 
Maharachchikumbura SSN, Guo LD, Chukeatirote E, Ekachai C et al. 2011 - Pestalotiopsis morphology, phylogeny, biochemistry and diversity. Fungal Diversity 50, 167-187.

Maharachchikumbura SSN, Guo LD, Cai L, Ekachai C et al. 2012 - A multi-locus backbone tree for Pestalotiopsis, with a polyphasic characterization of 14 new species. Fungal Diversity 56, 95-129.

Maharachchikumbura SSN, Guo LD, Chukeatirote E, McKenzie EHC, Hyde KD. 2013 - A destructive new disease of Syzygium samarangense in Thailand caused by the new species Pestalotiopsis samarangensis. Tropical Plant Pathology 38, 227-235.

Maharachchikumbura SSN, Hyde KD, Groenewald JZ, Xu J, Crous PW. 2014 - Pestalotiopsis revisited. Studies Mycology 79, 121-186.

Maharachchikumbura SSN, Guo LD, Liu ZY, Hyde KD. 2016 - Pseudopestalotiopsisignota and Ps. camelliae spp. nov. associated with grey blight disease of tea in China. Mycological Progress $15,22$.

McMillan Jr RT. 1964 - Studies of a recently described Cercospora on Rhizophora mangle. Plant Disease Reporter 48, 909-911.

Nag Raj TR. 1993 - Coelomycetous anamorphs with appendage-bearing conidia. Mycologue publications, Waterloo, 1-1101.

Norphanphoun C, Doilom M, Daranagama DA, Phookamsak R et al. 2017 - Revisiting the genus Cytospora and allied species. Mycosphere 8, 51-97.

Norphanphoun C, Raspé O, Jeewon R, Wen TC, Hyde KD. 2018 -Morphological and phylogenetic characterisation of novel Cytospora species associated with mangroves. MycoKeys 38, 93-120.

Nozawa S, Yamaguchi K, Van Hop D, Phay N et al. 2017 - Identification of two new species and asexual morph from the genus Pseudopestalotiopsis. Mycoscience 58, 328-337.

Nozawa S, Ando K, Phay N, Watanabe K. 2018 - Pseudopestalotiopsis dawaina sp. nov. and Ps. kawthaungina sp. nov.: two new species from Myanmar. Mycological Progress 17, 865-870.

Nylander JAA. 2004 - MrModeltest 2.0. Program distributed by the author. Evolutionary Biology Centre, Uppsala University.

O'Donnell K, Cigelnik E. 1997 - Two divergent intragenomic rDNA ITS2 types within a monophyletic lineage of the fungus Fusarium are nonorthologous. Molecular Phylogenetics and Evolution 7, 103-116.

O'Donnell K, Kistler HC, Cigelnik E, Ploetz RC. 1998 - Multiple evolutionary origins of the fungus causing Panama disease of banana: concordant evidence from nuclear and mitochondrial gene genealogies. Proceedings of the National Academy of Sciences of the United States of America 95, 2044-2049.

Pang KL, Alias SA, Chiang MWL, Vrijmoed LLP. 2010 - 'Sedecimiella taiwanensis gen. et sp. nov., a marine mangrove fungus in the Hypocreales (Hypocreomycetidae, Ascomycota)'. Botanica Marina 53, 493-498.

Rambaut A, Suchard MA, Xie D, Drummond AJ. 2013 - Tracer v1. 6, http://beast.bio.ed.ac.uk/software/tracer/ (Accessed April 2019).

Rambaut A. 2014 -FigTree v1. 4: Tree Figure drawing tool. http: / / treebio.ed.ac.uk/software/figtree/

Ronquist F, Teslenko M, van der Mark P, Ayres DL et al. 2012 - MrBayes 3.2: efficient Bayesian phylogenetic inference and model choice across a large model space. Syst Biol 61, 539-542.

Ryvarden L. 2000 - 'Studies in neotropical polypores 2: a preliminary key to neotropical species of Ganoderma with a laccate pileus'. Mycologia 92, 180-191.

Sakayaroj J, Pang KL, Jones EBG. 2012 - 'Multigene phylogeny of the Halosphaeriaceae: Its ordinal status, relationships between genera and morphological character evolution'. Fungal Diversity 46, 87-109.

Shivas RG, Young AJ, Crous PW. 2009 - Pseudocercospora avicenniae. Fungal Planet 40. Persoonia 23, 192-193. 
Song Y, Geng K, Zhang B, Hyde KD et al. 2013 - Two new species of Pestalotiopsis from Southern China. Phytotaxa 126, 22-30.

Song Y, Tangthirasunun N, Maharachchikumbura SSN, Jiang Y et al. 2014 - Novel Pestalotiopsis species from Thailand point to the rich undiscovered diversity of this chemically creative genus. Cryptogamie Mycologie 35, 139-149.

Stamatakis A. 2006 - RAxML-VI-HPC: maximum likelihood - based phylogenetic analyses with thousands of taxa and mixed models. Bioinformatics 22, 2688-2690.

Stevens FL 1920 - New or noteworthy Puerto Rican fungi. Botanical Gazette 70, 399-402.

Swofford DL. 2002 - PAUP: phylogenetic analysis using parsimony, (* and other methods). Version 4.0 b10. Sunderland, MA, USA: Sinauer Associates. https://doi.org/10.1111/j.00143820.2002.tb00191.x

Talavera G, Castresana J. 2007 - Improvement of phylogenies after removing divergent and ambiguously aligned blocks from protein sequence alignments. Systematic Biology 56, 564577.

Thanoi H, Behera BC, Mishra RR, Dutta SK. 2012 - Biodiversity and biotechnological potential of microorganisms from mangrove ecosystems: a review. Annals of Microbiology 63, 1-19.

Thatoi H, Behera BC, Mishra RR. 2013 - Ecological role and biotechnological potential of mangrove fungi: a review. Mycology 4, 54-71.

Thomas SAL, Fleming R, Shaw LN, Baker BJ. 2016 - Isolation of bioactive secondary metabolites from mangrove fungal endophytes using epigenetic regulation. Planta Med. 82, S1-S381.

Tibpromma S, Hyde KD, McKenzie EHC, Bhat DJ et al. 2018 - Fungal diversity notes 840-928: micro-fungi associated with Pandanaceae. Fungal Diversity 92, 1-160.

Tsai I, Maharachchikumbura SSN, Hyde KD, Ariyawansa HA. 2018 - Molecular phylogeny, morphology and pathogenicity of Pseudopestalotiopsis species of Ixora in Taiwan. Mycological Progress 17, 941-952.

Vollbrecht G, Johansson U, Eriksson H, Stenlid J. 1995 - 'Butt rot incidence, yield and growth pattern in a tree species experiment in southwestern Sweden'. Forest Ecological Management 76, 87-93.

Watanabe K, Nozawa S, Hsiang T, Callan B. 2018 - The cup fungus Pestalopezia brunneopruinosa is Pestalotiopsis gibbosa and belongs to Sordariomycetes. PLoS ONE 13, e0197025.

White T, Bruns T, Lee S, Taylor J. 1990 - Amplification and direct sequencing of fungal ribosomal RNA genes for phylogenetics. In: Innis MA, Gelfand DH, Sninsky JJ, White TJ, Eds., PCR Protocols: A Guide to Methods and Applications, Academic Press, Inc., New York, 315-322.

$\mathrm{Xu}$ J, Kjer J, Sendker J, Wray V et al. 2009 - Chromones from the endophytic fungus Pestalotiopsis sp. isolated from the Chinese mangrove plant Rhizophora mucronata. Journal of Natural Products 72, 662-5.

Zhou J, Diao X, Wang T, Chen G et al. 2018 - Phylogenetic diversity and antioxidant activities of culturable fungal endophytes associated with the mangrove species Rhizophora stylosa and $R$. mucronata in the South China Sea. PLoS ONE 13, e0197359. 
Supplementary Table 1 Nucleotides differences in the ITS, $\beta$-tubulin and EF1 $\alpha$ sequences of Neopestalotiopsis discussed in the paper.

\begin{tabular}{|c|c|c|c|c|c|c|c|c|c|c|c|c|c|c|c|}
\hline \multirow{2}{*}{ Taxon } & \multirow{2}{*}{ Strain } & \multicolumn{10}{|l|}{ ITS } & \multicolumn{4}{|c|}{$\beta$-tubulin } \\
\hline & & 68 & 85 & 100 & 370 & 372 & 373 & 374 & 375 & 449 & 487 & 163 & 169 & 201 & 205 \\
\hline Neopestalotiopsis acrostichi & MFLUCC 17-1754 & $\mathrm{T}$ & $\mathrm{A}$ & $\mathrm{T}$ & $\mathrm{T}$ & $\mathrm{C}$ & $\mathrm{A}$ & $\mathrm{T}$ & $\mathrm{T}$ & $\mathrm{T}$ & $\mathrm{T}$ & $\mathrm{C}$ & $\mathrm{T}$ & $\mathrm{T}$ & $\mathrm{A}$ \\
\hline N. alpapicalis & MFLUCC 17-2544 & - & $\mathrm{A}$ & $\mathrm{T}$ & $\mathrm{T}$ & - & - & $\mathrm{A}$ & $\mathrm{T}$ & $\mathrm{T}$ & $\mathrm{T}$ & - & - & - & - \\
\hline N. aotearoa & CBS 367.54 & $\mathrm{~T}$ & $\mathrm{G}$ & $\mathrm{T}$ & $\mathrm{T}$ & - & - & $\mathrm{A}$ & $\mathrm{T}$ & $\mathrm{T}$ & $\mathrm{T}$ & $\mathrm{C}$ & $\mathrm{T}$ & $\mathrm{C}$ & $\mathrm{T}$ \\
\hline N. brachiata & MFLUCC 17-1555 & $\mathrm{T}$ & $\mathrm{A}$ & $\mathrm{T}$ & $\mathrm{T}$ & - & - & $\mathrm{A}$ & $\mathrm{T}$ & $\mathrm{T}$ & $\mathrm{T}$ & $\mathrm{T}$ & $\mathrm{T}$ & $\mathrm{C}$ & $\mathrm{T}$ \\
\hline N. coffea-arabicae & HGUP4015 & $\mathrm{T}$ & $\mathrm{A}$ & $\mathrm{T}$ & $\mathrm{T}$ & - & - & $\mathrm{A}$ & $\mathrm{T}$ & $\mathrm{T}$ & $\mathrm{T}$ & - & - & - & - \\
\hline N. ellipsospora & MFLUCC 12-0283 & $\mathrm{T}$ & $\mathrm{A}$ & $\mathrm{T}$ & $\mathrm{C}$ & - & - & $\mathrm{G}$ & $\mathrm{G}$ & $\mathrm{T}$ & $\mathrm{T}$ & - & - & - & - \\
\hline N. petila & MFLUCC 17-1737 & $\mathrm{T}$ & $\mathrm{A}$ & $\mathrm{T}$ & $\mathrm{T}$ & - & - & $\mathrm{A}$ & $\mathrm{T}$ & $\mathrm{T}$ & $\mathrm{T}$ & $\mathrm{T}$ & $\mathrm{T}$ & $\mathrm{C}$ & $\mathrm{T}$ \\
\hline N. piceana & CBS 394.48 & $\mathrm{~T}$ & $\mathrm{G}$ & $\mathrm{T}$ & $\mathrm{T}$ & - & - & $\mathrm{A}$ & $\mathrm{T}$ & $\mathrm{T}$ & $\mathrm{T}$ & $\mathrm{T}$ & $\mathrm{T}$ & $\mathrm{C}$ & $\mathrm{T}$ \\
\hline N. protearum & CBS 114178 & $\mathrm{~T}$ & $\mathrm{~A}$ & $\mathrm{~T}$ & $\mathrm{C}$ & - & - & $\mathrm{G}$ & $\mathrm{G}$ & $\mathrm{T}$ & $\mathrm{C}$ & $\mathrm{C}$ & $\mathrm{T}$ & $\mathrm{T}$ & $\mathrm{A}$ \\
\hline N. rhizophorae & MFLUCC 17-1550 & $\mathrm{T}$ & $\mathrm{A}$ & $\mathrm{T}$ & $\mathrm{T}$ & - & - & $\mathrm{A}$ & $\mathrm{T}$ & $\mathrm{T}$ & $\mathrm{T}$ & $\mathrm{T}$ & $\mathrm{T}$ & $\mathrm{C}$ & $\mathrm{T}$ \\
\hline N. rosicola & CFCC 51992 & $\mathrm{~T}$ & $\mathrm{~A}$ & $\mathrm{~T}$ & $\mathrm{~T}$ & - & - & $\mathrm{T}$ & $\mathrm{A}$ & $\mathrm{C}$ & $\mathrm{T}$ & $\mathrm{T}$ & $\mathrm{T}$ & $\mathrm{C}$ & $\mathrm{T}$ \\
\hline N. samarangensis & MFLUCC 12-0233 & $\mathrm{T}$ & $\mathrm{A}$ & $\mathrm{T}$ & $\mathrm{T}$ & - & - & $\mathrm{A}$ & $\mathrm{T}$ & $\mathrm{T}$ & $\mathrm{T}$ & - & - & - & - \\
\hline N. sonneratae & MFLUCC 17-1744 & $\mathrm{T}$ & $\mathrm{A}$ & $\mathrm{T}$ & $\mathrm{T}$ & - & - & $\mathrm{A}$ & $\mathrm{T}$ & $\mathrm{T}$ & $\mathrm{T}$ & $\mathrm{C}$ & $\mathrm{T}$ & $\mathrm{T}$ & $\mathrm{A}$ \\
\hline N. surinamensis & CBS 450.74 & $\mathrm{~T}$ & $\mathrm{~A}$ & $\mathrm{~T}$ & $\mathrm{~T}$ & - & - & $\mathrm{T}$ & $\mathrm{T}$ & $\mathrm{T}$ & $\mathrm{T}$ & $\mathrm{C}$ & $\mathrm{A}$ & $\mathrm{T}$ & $\mathrm{A}$ \\
\hline N. thailandica & MFLUCC 17-1730 & $\mathrm{T}$ & A & $\mathrm{C}$ & $\mathrm{T}$ & - & - & $\mathrm{A}$ & $\mathrm{T}$ & $\mathrm{T}$ & $\mathrm{T}$ & $\mathrm{C}$ & $\mathrm{T}$ & $\mathrm{T}$ & A \\
\hline \multirow{2}{*}{ Taxon } & \multirow{2}{*}{ Strain } & \multicolumn{14}{|c|}{$\beta$-tubulin } \\
\hline & & 218 & 341 & 355 & 364 & 369 & 370 & 373 & 374 & 375 & 376 & 378 & 379 & 382 & 383 \\
\hline Neopestalotiopsis acrostichi & MFLUCC 17-1754 & $\mathrm{G}$ & $\mathrm{C}$ & $\mathrm{C}$ & $\mathrm{C}$ & $\mathrm{G}$ & $\mathrm{G}$ & $\mathrm{T}$ & $\mathrm{G}$ & $\mathrm{T}$ & $\mathrm{T}$ & $\mathrm{C}$ & $\mathrm{C}$ & $\mathrm{T}$ & $\mathrm{C}$ \\
\hline N. alpapicalis & MFLUCC 17-2544 & - & - & $\mathrm{C}$ & $\mathrm{T}$ & $\mathrm{G}$ & $\mathrm{G}$ & $\mathrm{T}$ & $\mathrm{G}$ & $\mathrm{T}$ & $\mathrm{A}$ & $\mathrm{T}$ & $\mathrm{C}$ & $\mathrm{T}$ & $\mathrm{C}$ \\
\hline N. aotearoa & CBS 367.54 & $\mathrm{~T}$ & $\mathrm{C}$ & $\mathrm{T}$ & $\mathrm{C}$ & $\mathrm{G}$ & $\mathrm{G}$ & $\mathrm{T}$ & $\mathrm{G}$ & $\mathrm{T}$ & $\mathrm{A}$ & $\mathrm{T}$ & $\mathrm{C}$ & $\mathrm{T}$ & $\mathrm{C}$ \\
\hline N. brachiata & MFLUCC 17-1555 & $\mathrm{T}$ & $\mathrm{C}$ & $\mathrm{T}$ & $\mathrm{C}$ & $\mathrm{G}$ & $\mathrm{G}$ & $\mathrm{T}$ & $\mathrm{G}$ & $\mathrm{T}$ & $\mathrm{A}$ & $\mathrm{T}$ & $\mathrm{C}$ & $\mathrm{T}$ & $\mathrm{C}$ \\
\hline N. coffea-arabicae & HGUP4015 & - & - & - & - & $\mathrm{A}$ & $\mathrm{A}$ & $\mathrm{C}$ & $\mathrm{A}$ & $\mathrm{C}$ & $\mathrm{A}$ & $\mathrm{C}$ & $\mathrm{G}$ & $\mathrm{A}$ & $\mathrm{T}$ \\
\hline N. ellipsospora & MFLUCC 12-0283 & - & - & $\mathrm{C}$ & $\mathrm{T}$ & $\mathrm{G}$ & $\mathrm{G}$ & $\mathrm{T}$ & $\mathrm{G}$ & $\mathrm{T}$ & $\mathrm{A}$ & $\mathrm{T}$ & $\mathrm{C}$ & $\mathrm{T}$ & $\mathrm{C}$ \\
\hline N. petila & MFLUCC 17-1737 & $\mathrm{T}$ & $\mathrm{C}$ & $\mathrm{T}$ & $\mathrm{C}$ & $\mathrm{G}$ & $\mathrm{G}$ & $\mathrm{T}$ & $\mathrm{G}$ & $\mathrm{T}$ & $\mathrm{A}$ & $\mathrm{T}$ & $\mathrm{C}$ & $\mathrm{T}$ & $\mathrm{C}$ \\
\hline N. piceana & CBS 394.48 & $\mathrm{~T}$ & $\mathrm{C}$ & $\mathrm{T}$ & $\mathrm{C}$ & $\mathrm{G}$ & $\mathrm{G}$ & $\mathrm{T}$ & $\mathrm{G}$ & $\mathrm{T}$ & $\mathrm{A}$ & $\mathrm{T}$ & $\mathrm{C}$ & $\mathrm{T}$ & $\mathrm{C}$ \\
\hline N. protearum & CBS 114178 & $\mathrm{G}$ & $\mathrm{C}$ & $\mathrm{C}$ & $\mathrm{C}$ & $\mathrm{G}$ & $\mathrm{G}$ & $\mathrm{T}$ & $\mathrm{G}$ & $\mathrm{T}$ & $\mathrm{T}$ & $\mathrm{C}$ & $\mathrm{C}$ & $\mathrm{T}$ & $\mathrm{C}$ \\
\hline N. rhizophorae & MFLUCC 17-1550 & $\mathrm{T}$ & $\mathrm{C}$ & $\mathrm{T}$ & $\mathrm{C}$ & $\mathrm{G}$ & $\mathrm{G}$ & $\mathrm{T}$ & $\mathrm{G}$ & $\mathrm{T}$ & $\mathrm{A}$ & $\mathrm{T}$ & $\mathrm{C}$ & $\mathrm{T}$ & $\mathrm{C}$ \\
\hline N. rosicola & CFCC 51992 & $\mathrm{~T}$ & $\mathrm{C}$ & $\mathrm{T}$ & $\mathrm{C}$ & $\mathrm{G}$ & $\mathrm{G}$ & $\mathrm{T}$ & $\mathrm{G}$ & $\mathrm{T}$ & A & $\mathrm{T}$ & $\mathrm{C}$ & $\mathrm{T}$ & $\mathrm{C}$ \\
\hline N. samarangensis & MFLUCC 12-0233 & - & - & $\mathrm{C}$ & $\mathrm{T}$ & $\mathrm{G}$ & $\mathrm{G}$ & $\mathrm{T}$ & $\mathrm{G}$ & $\mathrm{T}$ & $\mathrm{A}$ & $\mathrm{T}$ & $\mathrm{C}$ & $\mathrm{T}$ & $\mathrm{C}$ \\
\hline N. sonneratae & MFLUCC 17-1744 & $\mathrm{T}$ & $\mathrm{T}$ & $\mathrm{C}$ & $\mathrm{C}$ & $\mathrm{G}$ & $\mathrm{G}$ & $\mathrm{T}$ & $\mathrm{G}$ & $\mathrm{T}$ & $\mathrm{T}$ & $\mathrm{C}$ & $\mathrm{C}$ & $\mathrm{T}$ & $\mathrm{C}$ \\
\hline N. surinamensis & CBS 450.74 & $\mathrm{~T}$ & $\mathrm{C}$ & $\mathrm{C}$ & $\mathrm{C}$ & $\mathrm{G}$ & $\mathrm{G}$ & $\mathrm{T}$ & $\mathrm{G}$ & $\mathrm{T}$ & $\mathrm{T}$ & $\mathrm{C}$ & $\mathrm{C}$ & $\mathrm{T}$ & $\mathrm{C}$ \\
\hline N. thailandica & MFLUCC 17-1730 & $\mathrm{T}$ & $\mathrm{T}$ & $\mathrm{C}$ & $\mathrm{C}$ & $\mathrm{G}$ & G & $\mathrm{T}$ & G & $\mathrm{T}$ & $\mathrm{T}$ & $\mathrm{C}$ & $\mathrm{C}$ & $\mathrm{T}$ & $\mathrm{C}$ \\
\hline
\end{tabular}


Supplementary Table 1 Continued.

\begin{tabular}{|c|c|c|c|c|c|c|c|c|c|c|c|c|c|c|c|}
\hline \multirow{2}{*}{ Taxon } & \multirow{2}{*}{ Strain } & \multicolumn{14}{|c|}{$\beta$-tubulin } \\
\hline & & 384 & 385 & 386 & 393 & 402 & 428 & 437 & 438 & 441 & 446 & 459 & 469 & 471 & 472 \\
\hline Neopestalotiopsis acrostichi & MFLUCC 17-1754 & $\mathrm{T}$ & $\mathrm{G}$ & $\mathrm{C}$ & $\mathrm{A}$ & $\mathrm{C}$ & $\mathrm{C}$ & $\mathrm{A}$ & $\mathrm{C}$ & - & $\mathrm{A}$ & $\mathrm{G}$ & $\mathrm{G}$ & $\mathrm{T}$ & $\mathrm{A}$ \\
\hline N. alpapicalis & MFLUCC 17-2544 & $\mathrm{T}$ & $\mathrm{G}$ & $\mathrm{C}$ & $\mathrm{A}$ & $\mathrm{A}$ & $\mathrm{C}$ & $\mathrm{T}$ & $\mathrm{C}$ & - & $\mathrm{T}$ & $\mathrm{C}$ & $\mathrm{G}$ & $\mathrm{T}$ & $\mathrm{G}$ \\
\hline N. aotearoa & CBS 367.54 & $\mathrm{~T}$ & $\mathrm{G}$ & $\mathrm{C}$ & $\mathrm{A}$ & $\mathrm{A}$ & $\mathrm{C}$ & $\mathrm{T}$ & $\mathrm{C}$ & - & $\mathrm{T}$ & $\mathrm{C}$ & $\mathrm{G}$ & $\mathrm{T}$ & $\mathrm{G}$ \\
\hline N. brachiata & MFLUCC 17-1555 & $\mathrm{T}$ & $\mathrm{G}$ & $\mathrm{C}$ & $\mathrm{A}$ & $\mathrm{A}$ & $\mathrm{C}$ & $\mathrm{T}$ & $\mathrm{C}$ & - & $\mathrm{T}$ & $\mathrm{C}$ & $\mathrm{G}$ & $\mathrm{T}$ & $\mathrm{G}$ \\
\hline N. coffea-arabicae & HGUP4015 & $\mathrm{C}$ & $\mathrm{T}$ & $\mathrm{G}$ & - & $\mathrm{A}$ & $\mathrm{C}$ & $\mathrm{A}$ & $\mathrm{C}$ & $\mathrm{T}$ & $\mathrm{A}$ & $\mathrm{G}$ & $\mathrm{A}$ & $\mathrm{G}$ & $\mathrm{A}$ \\
\hline N. ellipsospora & MFLUCC 12-0283 & $\mathrm{T}$ & $\mathrm{G}$ & $\mathrm{C}$ & $\mathrm{A}$ & $\mathrm{A}$ & $\mathrm{T}$ & $\mathrm{T}$ & $\mathrm{C}$ & - & $\mathrm{T}$ & $\mathrm{C}$ & $\mathrm{G}$ & $\mathrm{T}$ & $\mathrm{G}$ \\
\hline N. petila & MFLUCC 17-1737 & $\mathrm{T}$ & $\mathrm{G}$ & $\mathrm{C}$ & $\mathrm{A}$ & $\mathrm{A}$ & $\mathrm{C}$ & $\mathrm{T}$ & $\mathrm{C}$ & - & $\mathrm{T}$ & $\mathrm{C}$ & $\mathrm{G}$ & $\mathrm{T}$ & $\mathrm{G}$ \\
\hline N. piceana & CBS 394.48 & $\mathrm{~T}$ & $\mathrm{G}$ & $\mathrm{C}$ & $\mathrm{A}$ & $\mathrm{A}$ & $\mathrm{C}$ & $\mathrm{T}$ & $\mathrm{C}$ & - & $\mathrm{T}$ & $\mathrm{C}$ & $\mathrm{G}$ & $\mathrm{T}$ & $\mathrm{G}$ \\
\hline N. protearum & CBS 114178 & $\mathrm{~T}$ & $\mathrm{G}$ & $\mathrm{C}$ & $\mathrm{A}$ & $\mathrm{C}$ & $\mathrm{C}$ & $\mathrm{A}$ & $\mathrm{A}$ & - & $\mathrm{A}$ & $\mathrm{G}$ & $\mathrm{G}$ & $\mathrm{T}$ & $\mathrm{A}$ \\
\hline N. rhizophorae & MFLUCC 17-1550 & $\mathrm{T}$ & $\mathrm{G}$ & $\mathrm{C}$ & $\mathrm{A}$ & $\mathrm{A}$ & $\mathrm{C}$ & $\mathrm{T}$ & $\mathrm{C}$ & - & $\mathrm{T}$ & $\mathrm{C}$ & $\mathrm{G}$ & $\mathrm{T}$ & $\mathrm{G}$ \\
\hline N. rosicola & CFCC 51992 & $\mathrm{~T}$ & $\mathrm{G}$ & $\mathrm{C}$ & $\mathrm{A}$ & $\mathrm{A}$ & $\mathrm{C}$ & $\mathrm{T}$ & $\mathrm{C}$ & - & $\mathrm{T}$ & $\mathrm{C}$ & $\mathrm{G}$ & $\mathrm{T}$ & $\mathrm{G}$ \\
\hline N. samarangensis & MFLUCC 12-0233 & $\mathrm{T}$ & $\mathrm{G}$ & $\mathrm{C}$ & $\mathrm{A}$ & A & $\mathrm{C}$ & $\mathrm{T}$ & $\mathrm{C}$ & - & $\mathrm{T}$ & $\mathrm{C}$ & $\mathrm{G}$ & $\mathrm{T}$ & $\mathrm{G}$ \\
\hline N. sonneratae & MFLUCC 17-1744 & $\mathrm{T}$ & $\mathrm{G}$ & $\mathrm{C}$ & $\mathrm{A}$ & $\mathrm{A}$ & $\mathrm{C}$ & $\mathrm{A}$ & $\mathrm{C}$ & $\mathrm{T}$ & $\mathrm{A}$ & $\mathrm{G}$ & $\mathrm{A}$ & $\mathrm{T}$ & $\mathrm{A}$ \\
\hline N. surinamensis & CBS 450.74 & $\mathrm{~T}$ & $\mathrm{G}$ & $\mathrm{C}$ & $\mathrm{A}$ & $\mathrm{C}$ & $\mathrm{C}$ & $\mathrm{A}$ & $\mathrm{C}$ & - & $\mathrm{A}$ & $\mathrm{G}$ & $\mathrm{G}$ & $\mathrm{T}$ & $\mathrm{A}$ \\
\hline N. thailandica & MFLUCC 17-1730 & $\mathrm{T}$ & $\mathrm{G}$ & $\mathrm{C}$ & $\mathrm{A}$ & $\mathrm{A}$ & $\mathrm{C}$ & $\mathrm{A}$ & $\mathrm{C}$ & $\mathrm{T}$ & A & $\mathrm{G}$ & A & $\mathrm{G}$ & A \\
\hline \multirow{2}{*}{ Taxon } & \multirow{2}{*}{ Strain } & \multicolumn{5}{|c|}{$\beta$-tubulin } & \multicolumn{9}{|l|}{ EF1a } \\
\hline & & 543 & 544 & 571 & 674 & 683 & 70 & 81 & 84 & 85 & 86 & 87 & 88 & 89 & 90 \\
\hline Neopestalotiopsis acrostichi & MFLUCC 17-1754 & $\mathrm{A}$ & $\mathrm{C}$ & $\mathrm{T}$ & $\mathrm{T}$ & $\mathrm{C}$ & $\mathrm{T}$ & $\mathrm{C}$ & $\mathrm{C}$ & A & $\mathrm{T}$ & - & - & - & - \\
\hline N. alpapicalis & MFLUCC 17-2544 & $\mathrm{T}$ & $\mathrm{T}$ & $\mathrm{C}$ & $\mathrm{T}$ & $\mathrm{C}$ & - & - & $\mathrm{C}$ & $\mathrm{A}$ & $\mathrm{T}$ & - & - & - & - \\
\hline N. aotearoa & CBS 367.54 & $\mathrm{~T}$ & $\mathrm{~T}$ & $\mathrm{C}$ & $\mathrm{T}$ & $\mathrm{C}$ & $\mathrm{T}$ & $\mathrm{C}$ & $\mathrm{C}$ & $\mathrm{A}$ & $\mathrm{T}$ & - & - & - & - \\
\hline N. brachiata & MFLUCC 17-1555 & $\mathrm{T}$ & $\mathrm{T}$ & $\mathrm{C}$ & $\mathrm{T}$ & $\mathrm{C}$ & $\mathrm{T}$ & $\mathrm{C}$ & $\mathrm{C}$ & $\mathrm{A}$ & $\mathrm{T}$ & - & - & - & - \\
\hline N. coffea-arabicae & HGUP4015 & $\mathrm{A}$ & $\mathrm{C}$ & $\mathrm{C}$ & $\mathrm{T}$ & $\mathrm{T}$ & $\mathrm{T}$ & $\mathrm{C}$ & $\mathrm{C}$ & $\mathrm{A}$ & $\mathrm{T}$ & - & - & - & - \\
\hline N. ellipsospora & MFLUCC 12-0283 & $\mathrm{T}$ & $\mathrm{T}$ & $\mathrm{C}$ & $\mathrm{T}$ & $\mathrm{C}$ & $\mathrm{T}$ & $\mathrm{A}$ & $\mathrm{C}$ & $\mathrm{A}$ & $\mathrm{T}$ & - & - & - & - \\
\hline N. petila & MFLUCC 17-1737 & $\mathrm{T}$ & $\mathrm{T}$ & $\mathrm{C}$ & $\mathrm{C}$ & $\mathrm{C}$ & $\mathrm{T}$ & $\mathrm{C}$ & $\mathrm{C}$ & $\mathrm{A}$ & $\mathrm{T}$ & - & - & - & - \\
\hline N. piceana & CBS 394.48 & $\mathrm{~T}$ & $\mathrm{~T}$ & $\mathrm{C}$ & $\mathrm{T}$ & $\mathrm{C}$ & $\mathrm{T}$ & $\mathrm{C}$ & $\mathrm{C}$ & $\mathrm{A}$ & $\mathrm{T}$ & - & - & - & - \\
\hline N. protearum & CBS 114178 & $\mathrm{~A}$ & $\mathrm{C}$ & $\mathrm{T}$ & $\mathrm{T}$ & $\mathrm{C}$ & $\mathrm{T}$ & $\mathrm{C}$ & - & - & - & - & - & - & - \\
\hline N. rhizophorae & MFLUCC 17-1550 & $\mathrm{T}$ & $\mathrm{T}$ & $\mathrm{C}$ & $\mathrm{T}$ & $\mathrm{C}$ & $\mathrm{T}$ & $\mathrm{C}$ & $\mathrm{C}$ & $\mathrm{A}$ & $\mathrm{T}$ & - & - & - & - \\
\hline N. rosicola & CFCC 51992 & $\mathrm{~T}$ & $\mathrm{~T}$ & $\mathrm{C}$ & $\mathrm{T}$ & $\mathrm{C}$ & $\mathrm{T}$ & $\mathrm{C}$ & $\mathrm{C}$ & $\mathrm{A}$ & $\mathrm{T}$ & - & - & - & - \\
\hline N. samarangensis & MFLUCC 12-0233 & $\mathrm{T}$ & $\mathrm{T}$ & $\mathrm{C}$ & $\mathrm{C}$ & $\mathrm{C}$ & $\mathrm{T}$ & $\mathrm{C}$ & $\mathrm{C}$ & $\mathrm{A}$ & $\mathrm{T}$ & - & - & - & - \\
\hline N. sonneratae & MFLUCC 17-1744 & $\mathrm{A}$ & $\mathrm{C}$ & $\mathrm{C}$ & $\mathrm{T}$ & $\mathrm{T}$ & $\mathrm{T}$ & $\mathrm{C}$ & $\mathrm{C}$ & $\mathrm{A}$ & $\mathrm{T}$ & $\mathrm{C}$ & $\mathrm{A}$ & $\mathrm{T}$ & $\mathrm{C}$ \\
\hline N. surinamensis & CBS 450.74 & $\mathrm{~A}$ & $\mathrm{C}$ & $\mathrm{T}$ & $\mathrm{T}$ & $\mathrm{C}$ & $\mathrm{C}$ & $\mathrm{C}$ & $\mathrm{C}$ & $\mathrm{A}$ & $\mathrm{T}$ & - & - & - & - \\
\hline N. thailandica & MFLUCC 17-1730 & A & $\mathrm{C}$ & $\mathrm{C}$ & $\mathrm{T}$ & $\mathrm{T}$ & $\mathrm{T}$ & $\mathrm{C}$ & $\mathrm{C}$ & A & $\mathrm{T}$ & $\mathrm{C}$ & A & $\mathrm{T}$ & $\mathrm{C}$ \\
\hline
\end{tabular}


Supplementary Table 1 Continued.

\begin{tabular}{|c|c|c|c|c|c|c|c|c|c|c|c|c|c|c|c|}
\hline \multirow{2}{*}{ Taxon } & \multirow{2}{*}{ Strain } & \multicolumn{14}{|c|}{ EF1a } \\
\hline & & 91 & 92 & 93 & 97 & 104 & 105 & 112 & 113 & 114 & 118 & 120 & 124 & 133 & 167 \\
\hline Neopestalotiopsis acrostichi & MFLUCC 17-1754 & - & - & - & $\mathrm{C}$ & $\mathrm{C}$ & $\mathrm{A}$ & $\mathrm{C}$ & $\mathrm{A}$ & $\mathrm{T}$ & $\mathrm{A}$ & $\mathrm{T}$ & $\mathrm{T}$ & $\mathrm{T}$ & $\mathrm{T}$ \\
\hline N. alpapicalis & MFLUCC 17-2544 & - & - & - & $\mathrm{C}$ & $\mathrm{C}$ & $\mathrm{A}$ & - & - & - & $\mathrm{A}$ & $\mathrm{T}$ & $\mathrm{C}$ & $\mathrm{T}$ & $\mathrm{T}$ \\
\hline N. aotearoa & CBS 367.54 & - & - & - & $\mathrm{C}$ & $\mathrm{C}$ & $\mathrm{A}$ & $\mathrm{C}$ & $\mathrm{A}$ & $\mathrm{T}$ & $\mathrm{A}$ & $\mathrm{T}$ & $\mathrm{C}$ & $\mathrm{T}$ & $\mathrm{T}$ \\
\hline N. brachiata & MFLUCC 17-1555 & - & - & - & $\mathrm{C}$ & $\mathrm{C}$ & $\mathrm{A}$ & $\mathrm{C}$ & $\mathrm{A}$ & $\mathrm{T}$ & $\mathrm{A}$ & $\mathrm{A}$ & $\mathrm{C}$ & $\mathrm{T}$ & $\mathrm{T}$ \\
\hline N. coffea-arabicae & HGUP4015 & - & - & - & $\mathrm{C}$ & $\mathrm{C}$ & $\mathrm{A}$ & $\mathrm{C}$ & $\mathrm{A}$ & $\mathrm{T}$ & $\mathrm{A}$ & $\mathrm{T}$ & $\mathrm{C}$ & $\mathrm{T}$ & $\mathrm{T}$ \\
\hline N. ellipsospora & MFLUCC 12-0283 & - & - & - & $\mathrm{C}$ & $\mathrm{C}$ & $\mathrm{A}$ & $\mathrm{C}$ & $\mathrm{A}$ & $\mathrm{T}$ & $\mathrm{A}$ & $\mathrm{T}$ & $\mathrm{C}$ & $\mathrm{T}$ & $\mathrm{T}$ \\
\hline N. petila & MFLUCC 17-1737 & - & - & - & $\mathrm{C}$ & $\mathrm{C}$ & $\mathrm{A}$ & $\mathrm{C}$ & $\mathrm{A}$ & $\mathrm{T}$ & $\mathrm{A}$ & $\mathrm{T}$ & $\mathrm{C}$ & $\mathrm{T}$ & $\mathrm{T}$ \\
\hline N. piceana & CBS 394.48 & - & - & - & $\mathrm{C}$ & $\mathrm{C}$ & $\mathrm{A}$ & $\mathrm{C}$ & $\mathrm{A}$ & $\mathrm{T}$ & $\mathrm{A}$ & $\mathrm{T}$ & $\mathrm{C}$ & $\mathrm{T}$ & $\mathrm{G}$ \\
\hline N. protearum & CBS 114178 & - & - & - & $\mathrm{C}$ & $\mathrm{C}$ & $\mathrm{A}$ & $\mathrm{C}$ & $\mathrm{A}$ & $\mathrm{T}$ & $\mathrm{A}$ & $\mathrm{T}$ & $\mathrm{C}$ & $\mathrm{T}$ & $\mathrm{T}$ \\
\hline N. rhizophorae & MFLUCC 17-1550 & - & - & - & $\mathrm{C}$ & $\mathrm{C}$ & $\mathrm{A}$ & - & - & - & $\mathrm{A}$ & $\mathrm{T}$ & $\mathrm{C}$ & $\mathrm{T}$ & $\mathrm{T}$ \\
\hline N. rosicola & CFCC 51992 & - & - & - & $\mathrm{C}$ & $\mathrm{C}$ & $\mathrm{A}$ & $\mathrm{C}$ & $\mathrm{A}$ & $\mathrm{T}$ & $\mathrm{A}$ & $\mathrm{T}$ & $\mathrm{C}$ & $\mathrm{T}$ & $\mathrm{T}$ \\
\hline N. samarangensis & MFLUCC 12-0233 & - & - & - & $\mathrm{C}$ & $\mathrm{G}$ & $\mathrm{A}$ & $\mathrm{C}$ & $\mathrm{A}$ & $\mathrm{T}$ & $\mathrm{A}$ & $\mathrm{T}$ & $\mathrm{C}$ & $\mathrm{T}$ & $\mathrm{T}$ \\
\hline N. sonneratae & MFLUCC 17-1744 & $\mathrm{C}$ & $\mathrm{C}$ & $\mathrm{C}$ & $\mathrm{C}$ & $\mathrm{C}$ & $\mathrm{A}$ & $\mathrm{C}$ & $\mathrm{A}$ & $\mathrm{T}$ & $\mathrm{A}$ & $\mathrm{T}$ & $\mathrm{C}$ & $\mathrm{T}$ & $\mathrm{T}$ \\
\hline N. surinamensis & CBS 450.74 & - & - & - & $\mathrm{T}$ & $\mathrm{C}$ & $\mathrm{T}$ & $\mathrm{C}$ & $\mathrm{G}$ & $\mathrm{T}$ & $\mathrm{G}$ & $\mathrm{T}$ & $\mathrm{C}$ & $\mathrm{C}$ & $\mathrm{T}$ \\
\hline N. thailandica & MFLUCC 17-1730 & $\mathrm{C}$ & $\mathrm{C}$ & $\mathrm{C}$ & $\mathrm{C}$ & $\mathrm{C}$ & $\mathrm{A}$ & $\mathrm{C}$ & $\mathrm{A}$ & $\mathrm{T}$ & $\mathrm{A}$ & $\mathrm{T}$ & $\mathrm{C}$ & $\mathrm{T}$ & $\mathrm{T}$ \\
\hline \multirow{2}{*}{ Taxon } & \multirow{2}{*}{ Strain } & \multicolumn{14}{|c|}{ EF1a } \\
\hline & & 169 & 186 & 210 & 246 & 287 & 290 & 407 & 456 & 462 & 480 & 485 & & & \\
\hline Neopestalotiopsis acrostichi & MFLUCC 17-1754 & - & $\mathrm{G}$ & $\mathrm{G}$ & $\mathrm{G}$ & A & $\mathrm{T}$ & $\mathrm{T}$ & $\mathrm{C}$ & $\mathrm{A}$ & $\mathrm{A}$ & $\mathrm{A}$ & & & \\
\hline N. alpapicalis & MFLUCC 17-2544 & - & $\mathrm{G}$ & $\mathrm{G}$ & $\mathrm{G}$ & $\mathrm{A}$ & $\mathrm{T}$ & $\mathrm{C}$ & $\mathrm{T}$ & $\mathrm{A}$ & $\mathrm{A}$ & $\mathrm{G}$ & & & \\
\hline N. aotearoa & CBS 367.54 & - & $\mathrm{G}$ & $\mathrm{G}$ & $\mathrm{G}$ & $\mathrm{A}$ & $\mathrm{T}$ & $\mathrm{C}$ & $\mathrm{C}$ & $\mathrm{A}$ & $\mathrm{T}$ & $\mathrm{A}$ & & & \\
\hline N. brachiata & MFLUCC 17-1555 & - & $\mathrm{G}$ & $\mathrm{G}$ & $\mathrm{G}$ & $\mathrm{A}$ & $\mathrm{T}$ & $\mathrm{C}$ & $\mathrm{C}$ & $\mathrm{A}$ & $\mathrm{T}$ & $\mathrm{A}$ & & & \\
\hline N. coffea-arabicae & HGUP4015 & - & $\mathrm{G}$ & $\mathrm{G}$ & $\mathrm{G}$ & $\mathrm{A}$ & $\mathrm{T}$ & $\mathrm{C}$ & $\mathrm{C}$ & $\mathrm{A}$ & $\mathrm{T}$ & $\mathrm{A}$ & & & \\
\hline N. ellipsospora & MFLUCC 12-0283 & - & $\mathrm{A}$ & $\mathrm{A}$ & $\mathrm{A}$ & $\mathrm{A}$ & $\mathrm{T}$ & $\mathrm{C}$ & $\mathrm{C}$ & $\mathrm{A}$ & $\mathrm{T}$ & $\mathrm{A}$ & & & \\
\hline N. petila & MFLUCC 17-1737 & - & $\mathrm{G}$ & $\mathrm{G}$ & $\mathrm{G}$ & $\mathrm{A}$ & $\mathrm{T}$ & $\mathrm{C}$ & $\mathrm{C}$ & $\mathrm{A}$ & $\mathrm{T}$ & $\mathrm{A}$ & & & \\
\hline N. piceana & CBS 394.48 & - & $\mathrm{G}$ & $\mathrm{G}$ & $\mathrm{G}$ & $\mathrm{A}$ & $\mathrm{T}$ & $\mathrm{C}$ & $\mathrm{C}$ & $\mathrm{A}$ & $\mathrm{T}$ & $\mathrm{A}$ & & & \\
\hline$N$. protearum & CBS 114178 & $\mathrm{~T}$ & $\mathrm{G}$ & $\mathrm{G}$ & $\mathrm{G}$ & $\mathrm{A}$ & $\mathrm{T}$ & $\mathrm{C}$ & $\mathrm{C}$ & $\mathrm{A}$ & $\mathrm{T}$ & $\mathrm{A}$ & & & \\
\hline N. rhizophorae & MFLUCC 17-1550 & - & $\mathrm{G}$ & $\mathrm{G}$ & $\mathrm{G}$ & $\mathrm{A}$ & $\mathrm{T}$ & $\mathrm{C}$ & $\mathrm{C}$ & $\mathrm{A}$ & $\mathrm{A}$ & $\mathrm{G}$ & & & \\
\hline N. rosicola & CFCC 51992 & - & $\mathrm{G}$ & $\mathrm{G}$ & $\mathrm{G}$ & A & $\mathrm{T}$ & $\mathrm{C}$ & $\mathrm{C}$ & $\mathrm{A}$ & A & $\mathrm{A}$ & & & \\
\hline N. samarangensis & MFLUCC 12-0233 & - & $\mathrm{G}$ & $\mathrm{G}$ & $\mathrm{G}$ & $\mathrm{A}$ & $\mathrm{T}$ & $\mathrm{C}$ & $\mathrm{C}$ & $\mathrm{T}$ & $\mathrm{A}$ & $\mathrm{A}$ & & & \\
\hline N. sonneratae & MFLUCC 17-1744 & $\mathrm{C}$ & $\mathrm{G}$ & $\mathrm{G}$ & $\mathrm{G}$ & $\mathrm{A}$ & $\mathrm{C}$ & $\mathrm{C}$ & $\mathrm{C}$ & $\mathrm{T}$ & $\mathrm{A}$ & $\mathrm{A}$ & & & \\
\hline N. surinamensis & CBS 450.74 & - & $\mathrm{G}$ & $\mathrm{G}$ & $\mathrm{T}$ & $\mathrm{G}$ & $\mathrm{T}$ & $\mathrm{C}$ & $\mathrm{C}$ & $\mathrm{T}$ & $\mathrm{A}$ & $\mathrm{A}$ & & & \\
\hline N. thailandica & MFLUCC 17-1730 & $\mathrm{C}$ & $\mathrm{G}$ & $\mathrm{G}$ & $\mathrm{G}$ & $\mathrm{A}$ & $\mathrm{C}$ & $\mathrm{C}$ & $\mathrm{C}$ & $\mathrm{T}$ & $\mathrm{A}$ & $\mathrm{A}$ & & & \\
\hline
\end{tabular}


Supplementary Table 2 Nucleotides differences in the ITS, $\beta$-tubulin and EF1 $\alpha$ sequences of Pestalotiopsis discussed in the paper.

\begin{tabular}{|c|c|c|c|c|c|c|c|c|c|c|c|c|c|c|c|}
\hline \multirow{2}{*}{ Taxon } & \multirow{2}{*}{ Strain } & \multicolumn{11}{|l|}{ ITS } & \multicolumn{3}{|c|}{$\beta$-tubulin } \\
\hline & & 14 & 23 & 162 & 164 & 433 & 491 & 539 & 547 & 551 & 558 & 559 & 14 & 36 & 38 \\
\hline Pestalotiopsis rhizophorae & MFLUCC 17-0416 & $\mathrm{A}$ & $\mathrm{T}$ & $\mathrm{C}$ & $\mathrm{A}$ & $\mathrm{A}$ & $\mathrm{A}$ & $\mathrm{T}$ & $\mathrm{T}$ & $\mathrm{T}$ & $\mathrm{T}$ & $\mathrm{G}$ & $\mathrm{A}$ & $\mathrm{T}$ & $\mathrm{C}$ \\
\hline P. thailandica & MFLUCC 17-1616 & A & $\mathrm{T}$ & $\mathrm{C}$ & $\mathrm{G}$ & $\mathrm{G}$ & - & $\mathrm{C}$ & $\mathrm{T}$ & $\mathrm{T}$ & $\mathrm{T}$ & $\mathrm{G}$ & $\mathrm{T}$ & $\mathrm{T}$ & $\mathrm{C}$ \\
\hline P. formosana & NTUCC 17-009 & - & - & $\mathrm{T}$ & $\mathrm{G}$ & $\mathrm{A}$ & - & $\mathrm{C}$ & $\mathrm{T}$ & - & $\mathrm{G}$ & $\mathrm{T}$ & - & - & - \\
\hline P. parva & CBS 278.35 & $\mathrm{~T}$ & A & $\mathrm{T}$ & $\mathrm{G}$ & $\mathrm{A}$ & - & $\mathrm{C}$ & $\mathrm{C}$ & - & $\mathrm{T}$ & $\mathrm{G}$ & $\mathrm{A}$ & $\mathrm{G}$ & $\mathrm{G}$ \\
\hline \multirow{2}{*}{ Taxon } & \multirow{2}{*}{ Strain } & \multicolumn{14}{|c|}{$\beta$-tubulin } \\
\hline & & 39 & 43 & 130 & 131 & 132 & 153 & 174 & 260 & 360 & 368 & 372 & 403 & 428 & 430 \\
\hline Pestalotiopsis rhizophorae & MFLUCC 17-0416 & $\mathrm{C}$ & $\mathrm{C}$ & $\mathrm{A}$ & $\mathrm{A}$ & $\mathrm{G}$ & $\mathrm{C}$ & $\mathrm{C}$ & $\mathrm{C}$ & $\mathrm{C}$ & $\mathrm{T}$ & $\mathrm{A}$ & $\mathrm{G}$ & A & $\mathrm{T}$ \\
\hline P. thailandica & MFLUCC 17-1616 & $\mathrm{C}$ & $\mathrm{C}$ & $\mathrm{A}$ & $\mathrm{A}$ & $\mathrm{G}$ & $\mathrm{C}$ & $\mathrm{C}$ & $\mathrm{C}$ & $\mathrm{C}$ & $\mathrm{T}$ & $\mathrm{A}$ & $\mathrm{G}$ & A & $\mathrm{T}$ \\
\hline P. formosana & NTUCC 17-009 & - & - & - & - & - & - & - & - & - & - & $\mathrm{C}$ & $\mathrm{G}$ & A & $\mathrm{T}$ \\
\hline P. parva & CBS 278.35 & A & $\mathrm{C}$ & - & - & - & $\mathrm{G}$ & $\mathrm{T}$ & $\mathrm{A}$ & $\mathrm{T}$ & $\mathrm{C}$ & $\mathrm{A}$ & $\mathrm{A}$ & $\mathrm{T}$ & $\mathrm{C}$ \\
\hline \multirow{2}{*}{ Taxon } & \multirow{2}{*}{ Strain } & & \multicolumn{13}{|c|}{ EF1a } \\
\hline & & 762 & 33 & 34 & 36 & 56 & 57 & 58 & 71 & 81 & 123 & 126 & 226 & 261 & 434 \\
\hline Pestalotiopsis rhizophorae & MFLUCC 17-0416 & $\mathrm{A}$ & $\mathrm{T}$ & $\mathrm{C}$ & $\mathrm{C}$ & $\mathrm{C}$ & $\mathrm{C}$ & $\mathrm{A}$ & $\mathrm{C}$ & $\mathrm{C}$ & $\mathrm{A}$ & $\mathrm{C}$ & $\mathrm{T}$ & $\mathrm{C}$ & $\mathrm{G}$ \\
\hline P. thailandica & MFLUCC 17-1616 & $\mathrm{G}$ & $\mathrm{T}$ & $\mathrm{C}$ & $\mathrm{C}$ & $\mathrm{C}$ & $\mathrm{C}$ & $\mathrm{A}$ & $\mathrm{C}$ & $\mathrm{C}$ & $\mathrm{A}$ & $\mathrm{C}$ & $\mathrm{T}$ & $\mathrm{C}$ & $\mathrm{G}$ \\
\hline P. formosana & NTUCC 17-009 & $\mathrm{A}$ & $\mathrm{T}$ & $\mathrm{C}$ & $\mathrm{C}$ & - & - & - & $\mathrm{G}$ & $\mathrm{T}$ & $\mathrm{G}$ & $\mathrm{A}$ & $\mathrm{C}$ & $\mathrm{C}$ & A \\
\hline P. parva & CBS 278.35 & $\mathrm{~A}$ & $\mathrm{~A}$ & $\mathrm{~T}$ & $\mathrm{~T}$ & $\mathrm{~T}$ & $\mathrm{C}$ & $\mathrm{A}$ & $\mathrm{G}$ & $\mathrm{C}$ & $\mathrm{A}$ & $\mathrm{C}$ & $\mathrm{T}$ & $\mathrm{T}$ & $\mathrm{A}$ \\
\hline
\end{tabular}

Supplementary Table 3 Nucleotides differences in the ITS, $\beta$-tubulin and EF1 $\alpha$ sequences of Pseudopestalotiopsis discussed in the paper.

\begin{tabular}{|c|c|c|c|c|c|c|c|c|c|c|c|c|c|c|c|}
\hline \multirow{2}{*}{ Taxon } & \multirow{2}{*}{ Strain } & \multicolumn{14}{|l|}{ ITS } \\
\hline & & 30 & 39 & 75 & 119 & 147 & 409 & 410 & 482 & 527 & 545 & 546 & 549 & 553 & 554 \\
\hline Pseudopestalotiopsis avucenniae & MFLUCC 17-0434 & $\mathrm{A}$ & $\mathrm{T}$ & $\mathrm{A}$ & $\mathrm{G}$ & $\mathrm{A}$ & - & - & $\mathrm{T}$ & $\mathrm{T}$ & $\mathrm{G}$ & $\mathrm{A}$ & $\mathrm{A}$ & $\mathrm{G}$ & $\mathrm{C}$ \\
\hline Ps. curvatispora & MFLUCC 17-1722 & $\mathrm{A}$ & $\mathrm{T}$ & $\mathrm{A}$ & $\mathrm{G}$ & $\mathrm{G}$ & - & - & $\mathrm{C}$ & $\mathrm{T}$ & $\mathrm{G}$ & $\mathrm{A}$ & $\mathrm{A}$ & $\mathrm{G}$ & $\mathrm{C}$ \\
\hline Ps. dawaina & MM14 F0015 & $\mathrm{T}$ & A & $\mathrm{A}$ & $\mathrm{T}$ & $\mathrm{G}$ & - & - & $\mathrm{C}$ & $\mathrm{T}$ & $\mathrm{G}$ & A & $\mathrm{A}$ & $\mathrm{G}$ & $\mathrm{C}$ \\
\hline Ps. jiangxiensis & LC4479 & - & - & - & $\mathrm{G}$ & $\mathrm{A}$ & - & - & $\mathrm{T}$ & $\mathrm{T}$ & $\mathrm{G}$ & $\mathrm{A}$ & $\mathrm{A}$ & $\mathrm{G}$ & $\mathrm{C}$ \\
\hline Ps. kawthaungina & MM14 F0083 & $\mathrm{T}$ & $\mathrm{A}$ & $\mathrm{A}$ & $\mathrm{G}$ & $\mathrm{A}$ & - & - & $\mathrm{T}$ & $\mathrm{T}$ & $\mathrm{G}$ & $\mathrm{A}$ & $\mathrm{A}$ & $\mathrm{G}$ & $\mathrm{C}$ \\
\hline Ps. myanmarina & NBRC 112264 & $\mathrm{~T}$ & $\mathrm{~A}$ & $\mathrm{~A}$ & $\mathrm{G}$ & $\mathrm{A}$ & - & - & $\mathrm{T}$ & $\mathrm{T}$ & $\mathrm{G}$ & $\mathrm{A}$ & $\mathrm{A}$ & $\mathrm{G}$ & $\mathrm{C}$ \\
\hline Ps. rhizophorae & MFLUCC 17-560 & $\mathrm{A}$ & $\mathrm{T}$ & $\mathrm{A}$ & $\mathrm{G}$ & $\mathrm{G}$ & A & $\mathrm{A}$ & $\mathrm{C}$ & $\mathrm{T}$ & $\mathrm{G}$ & $\mathrm{A}$ & $\mathrm{A}$ & $\mathrm{G}$ & $\mathrm{C}$ \\
\hline Ps. simitheae & MFLUCC 12-0121 & $\mathrm{T}$ & $\mathrm{A}$ & $\mathrm{A}$ & $\mathrm{G}$ & $\mathrm{A}$ & - & - & $\mathrm{T}$ & - & - & - & $\mathrm{G}$ & $\mathrm{A}$ & $\mathrm{T}$ \\
\hline Ps. taiwanensis & NTUCC 17-002.1 & $\mathrm{T}$ & $\mathrm{A}$ & $\mathrm{A}$ & $\mathrm{G}$ & $\mathrm{A}$ & - & - & $\mathrm{T}$ & - & - & - & - & - & - \\
\hline Ps. thailandica & MFLUCC 17-1724 & $\mathrm{A}$ & $\mathrm{T}$ & A & $\mathrm{G}$ & $\mathrm{G}$ & $\mathrm{A}$ & $\mathrm{A}$ & $\mathrm{C}$ & $\mathrm{T}$ & $\mathrm{G}$ & $\mathrm{A}$ & $\mathrm{A}$ & $\mathrm{G}$ & $\mathrm{C}$ \\
\hline
\end{tabular}


Supplementary Table 3 Continued.

\begin{tabular}{|c|c|c|c|c|c|c|c|c|c|c|c|c|c|c|c|}
\hline \multirow{2}{*}{ Taxon } & \multirow{2}{*}{ Strain } & \multirow{2}{*}{$\begin{array}{c}\text { ITS } \\
556 \\
\end{array}$} & \multicolumn{13}{|c|}{$\beta$-tubulin } \\
\hline & & & 22 & 107 & 125 & 134 & 148 & 149 & 150 & 151 & 153 & 158 & 183 & 192 & 194 \\
\hline Pseudopestalotiopsis avucenniae & MFLUCC 17-0434 & $\mathrm{G}$ & $\mathrm{G}$ & $\mathrm{T}$ & $\mathrm{A}$ & $\mathrm{C}$ & $\mathrm{T}$ & - & - & - & $\mathrm{T}$ & $\mathrm{C}$ & $\mathrm{A}$ & $\mathrm{A}$ & $\mathrm{A}$ \\
\hline Ps. curvatispora & MFLUCC 17-1722 & $\mathrm{G}$ & $\mathrm{G}$ & $\mathrm{G}$ & $\mathrm{C}$ & $\mathrm{C}$ & $\mathrm{G}$ & $\mathrm{C}$ & $\mathrm{A}$ & $\mathrm{G}$ & $\mathrm{C}$ & $\mathrm{C}$ & $\mathrm{G}$ & $\mathrm{G}$ & $\mathrm{A}$ \\
\hline Ps. dawaina & MM14 F0015 & $\mathrm{G}$ & - & - & - & - & - & - & - & - & - & - & - & - & - \\
\hline Ps. jiangxiensis & LC4479 & $\mathrm{G}$ & $\mathrm{G}$ & $\mathrm{T}$ & $\mathrm{A}$ & $\mathrm{C}$ & $\mathrm{T}$ & - & - & - & $\mathrm{T}$ & $\mathrm{C}$ & $\mathrm{A}$ & $\mathrm{A}$ & $\mathrm{A}$ \\
\hline Ps. kawthaungina & MM14 F0083 & $\mathrm{G}$ & - & - & - & - & - & - & - & - & - & - & - & - & - \\
\hline Ps. myanmarina & NBRC 112264 & $\mathrm{G}$ & - & - & - & - & - & - & - & - & - & - & - & - & - \\
\hline Ps. rhizophorae & MFLUCC 17-560 & $\mathrm{G}$ & $\mathrm{A}$ & $\mathrm{T}$ & $\mathrm{C}$ & $\mathrm{T}$ & $\mathrm{G}$ & $\mathrm{C}$ & $\mathrm{A}$ & $\mathrm{G}$ & $\mathrm{T}$ & $\mathrm{A}$ & $\mathrm{A}$ & $\mathrm{G}$ & $\mathrm{G}$ \\
\hline Ps. simitheae & MFLUCC 12-0121 & $\mathrm{C}$ & - & - & - & - & - & - & - & - & - & - & - & - & - \\
\hline Ps. taiwanensis & NTUCC 17-002.1 & - & - & - & - & - & - & - & - & - & - & - & - & - & - \\
\hline Ps. thailandica & MFLUCC 17-1724 & $\mathrm{G}$ & $\mathrm{A}$ & $\mathrm{T}$ & $\mathrm{C}$ & $\mathrm{T}$ & $\mathrm{G}$ & - & - & - & $\mathrm{T}$ & $\mathrm{A}$ & $\mathrm{A}$ & $\mathrm{G}$ & $\mathrm{G}$ \\
\hline \multirow{2}{*}{ Taxon } & \multirow{2}{*}{ Strain } & \multicolumn{14}{|c|}{$\beta$-tubulin } \\
\hline & & 198 & 206 & 223 & 225 & 250 & 302 & 330 & 355 & 361 & 362 & 363 & 364 & 365 & 372 \\
\hline Pseudopestalotiopsis avucenniae & MFLUCC 17-0434 & $\mathrm{A}$ & $\mathrm{G}$ & $\mathrm{A}$ & $\mathrm{G}$ & $\mathrm{C}$ & $\mathrm{G}$ & $\mathrm{A}$ & $\mathrm{C}$ & $\mathrm{G}$ & $\mathrm{T}$ & $\mathrm{A}$ & $\mathrm{T}$ & $\mathrm{G}$ & $\mathrm{A}$ \\
\hline Ps. curvatispora & MFLUCC 17-1722 & $\mathrm{T}$ & $\mathrm{A}$ & $\mathrm{G}$ & A & $\mathrm{T}$ & $\mathrm{G}$ & $\mathrm{G}$ & $\mathrm{C}$ & $\mathrm{G}$ & $\mathrm{T}$ & A & $\mathrm{T}$ & $\mathrm{G}$ & $\mathrm{A}$ \\
\hline Ps. dawaina & MM14 F0015 & - & - & - & - & - & - & - & - & $\mathrm{G}$ & $\mathrm{T}$ & A & $\mathrm{T}$ & $\mathrm{G}$ & A \\
\hline Ps. jiangxiensis & LC4479 & $\mathrm{A}$ & $\mathrm{G}$ & A & $\mathrm{G}$ & $\mathrm{C}$ & $\mathrm{G}$ & $\mathrm{A}$ & $\mathrm{C}$ & $\mathrm{G}$ & $\mathrm{T}$ & $\mathrm{A}$ & $\mathrm{T}$ & $\mathrm{G}$ & $\mathrm{A}$ \\
\hline Ps. kawthaungina & MM14 F0083 & - & - & - & - & - & - & - & - & $\mathrm{G}$ & $\mathrm{T}$ & A & $\mathrm{T}$ & $\mathrm{G}$ & $\mathrm{A}$ \\
\hline Ps. myanmarina & NBRC 112264 & - & - & - & - & - & - & - & - & - & - & - & - & - & A \\
\hline Ps. rhizophorae & MFLUCC 17-560 & $\mathrm{T}$ & $\mathrm{A}$ & $\mathrm{A}$ & $\mathrm{A}$ & $\mathrm{T}$ & $\mathrm{A}$ & $\mathrm{A}$ & $\mathrm{C}$ & $\mathrm{G}$ & $\mathrm{T}$ & $\mathrm{A}$ & $\mathrm{T}$ & $\mathrm{G}$ & $\mathrm{A}$ \\
\hline Ps. simitheae & MFLUCC 12-0121 & - & - & - & - & - & - & - & - & $\mathrm{A}$ & $\mathrm{G}$ & $\mathrm{T}$ & $\mathrm{C}$ & $\mathrm{A}$ & - \\
\hline Ps. taiwanensis & NTUCC 17-002.1 & - & - & - & - & - & - & - & $\mathrm{T}$ & $\mathrm{G}$ & $\mathrm{T}$ & A & $\mathrm{T}$ & $\mathrm{G}$ & $\mathrm{A}$ \\
\hline Ps. thailandica & MFLUCC 17-1724 & $\mathrm{T}$ & $\mathrm{A}$ & $\mathrm{A}$ & $\mathrm{A}$ & $\mathrm{T}$ & $\mathrm{G}$ & A & $\mathrm{C}$ & $\mathrm{G}$ & $\mathrm{T}$ & A & $\mathrm{T}$ & $\mathrm{G}$ & $\mathrm{A}$ \\
\hline \multirow{2}{*}{ Taxon } & \multirow{2}{*}{ Strain } & \multicolumn{14}{|c|}{$\beta$-tubulin } \\
\hline & & 376 & 413 & 434 & 435 & 445 & 453 & 459 & 505 & 538 & 539 & 540 & 558 & 559 & 560 \\
\hline Pseudopestalotiopsis avucenniae & MFLUCC 17-0434 & $\mathrm{A}$ & $\mathrm{T}$ & $\mathrm{T}$ & $\mathrm{G}$ & $\mathrm{C}$ & - & $\mathrm{G}$ & $\mathrm{T}$ & - & - & - & $\mathrm{C}$ & $\mathrm{A}$ & $\mathrm{T}$ \\
\hline Ps. curvatispora & MFLUCC 17-1722 & $\mathrm{A}$ & $\mathrm{T}$ & $\mathrm{T}$ & $\mathrm{A}$ & $\mathrm{A}$ & $\mathrm{G}$ & $\mathrm{G}$ & $\mathrm{T}$ & $\mathrm{C}$ & $\mathrm{C}$ & $\mathrm{C}$ & $\mathrm{T}$ & $\mathrm{C}$ & $\mathrm{C}$ \\
\hline Ps. dawaina & MM14 F0015 & $\mathrm{A}$ & $\mathrm{T}$ & $\mathrm{T}$ & $\mathrm{A}$ & $\mathrm{A}$ & $\mathrm{G}$ & $\mathrm{G}$ & $\mathrm{T}$ & $\mathrm{C}$ & $\mathrm{C}$ & $\mathrm{C}$ & $\mathrm{T}$ & $\mathrm{C}$ & $\mathrm{C}$ \\
\hline Ps. jiangxiensis & LC4479 & A & $\mathrm{T}$ & $\mathrm{T}$ & $\mathrm{G}$ & $\mathrm{C}$ & - & $\mathrm{G}$ & $\mathrm{T}$ & - & - & - & $\mathrm{C}$ & A & $\mathrm{C}$ \\
\hline Ps. kawthaungina & MM14 F0083 & $\mathrm{A}$ & $\mathrm{T}$ & $\mathrm{T}$ & $\mathrm{A}$ & $\mathrm{A}$ & $\mathrm{G}$ & $\mathrm{G}$ & $\mathrm{T}$ & $\mathrm{C}$ & $\mathrm{C}$ & $\mathrm{C}$ & $\mathrm{T}$ & $\mathrm{C}$ & $\mathrm{C}$ \\
\hline Ps. myanmarina & NBRC 112264 & $\mathrm{~A}$ & $\mathrm{~T}$ & $\mathrm{~T}$ & $\mathrm{G}$ & $\mathrm{C}$ & - & $\mathrm{G}$ & $\mathrm{T}$ & - & - & - & $\mathrm{C}$ & $\mathrm{A}$ & $\mathrm{T}$ \\
\hline Ps. rhizophorae & MFLUCC 17-560 & $\mathrm{C}$ & $\mathrm{C}$ & $\mathrm{G}$ & $\mathrm{A}$ & $\mathrm{A}$ & $\mathrm{G}$ & $\mathrm{G}$ & $\mathrm{C}$ & $\mathrm{T}$ & $\mathrm{T}$ & $\mathrm{C}$ & $\mathrm{C}$ & $\mathrm{A}$ & $\mathrm{C}$ \\
\hline Ps. simitheae & MFLUCC 12-0121 & - & $\mathrm{C}$ & $\mathrm{G}$ & $\mathrm{A}$ & $\mathrm{A}$ & $\mathrm{G}$ & $\mathrm{G}$ & $\mathrm{T}$ & $\mathrm{T}$ & $\mathrm{T}$ & $\mathrm{C}$ & $\mathrm{C}$ & $\mathrm{A}$ & $\mathrm{C}$ \\
\hline Ps.taiwanensis & NTUCC 17-002.1 & $\mathrm{C}$ & $\mathrm{C}$ & $\mathrm{G}$ & $\mathrm{A}$ & $\mathrm{A}$ & $\mathrm{G}$ & $\mathrm{G}$ & $\mathrm{T}$ & $\mathrm{T}$ & $\mathrm{T}$ & $\mathrm{C}$ & $\mathrm{C}$ & $\mathrm{A}$ & $\mathrm{C}$ \\
\hline Ps. thailandica & MFLUCC 17-1724 & $\mathrm{C}$ & $\mathrm{C}$ & $\mathrm{G}$ & $\mathrm{A}$ & $\mathrm{A}$ & $\mathrm{G}$ & $\mathrm{A}$ & $\mathrm{C}$ & $\mathrm{T}$ & $\mathrm{T}$ & $\mathrm{C}$ & $\mathrm{C}$ & $\mathrm{A}$ & $\mathrm{C}$ \\
\hline
\end{tabular}


Supplementary Table 3 Continued.

\begin{tabular}{|c|c|c|c|c|c|c|c|c|c|c|c|c|c|c|c|}
\hline \multirow{2}{*}{ Taxon } & \multirow{2}{*}{ Strain } & \multicolumn{10}{|c|}{$\beta$-tubulin } & \multicolumn{4}{|c|}{ EF1a } \\
\hline & & 571 & 572 & 573 & 581 & 625 & 628 & 691 & 715 & 739 & 772 & 88 & 89 & 93 & 95 \\
\hline Pseudopestalotiopsis avucenniae & MFLUCC 17-0434 & $\mathrm{T}$ & $\mathrm{T}$ & $\mathrm{G}$ & $\mathrm{G}$ & $\mathrm{T}$ & $\mathrm{C}$ & $\mathrm{T}$ & $\mathrm{C}$ & $\mathrm{T}$ & $\mathrm{C}$ & $\mathrm{A}$ & $\mathrm{C}$ & $\mathrm{T}$ & $\mathrm{T}$ \\
\hline Ps. curvatispora & MFLUCC 17-1722 & $\mathrm{T}$ & $\mathrm{C}$ & $\mathrm{A}$ & $\mathrm{T}$ & $\mathrm{T}$ & $\mathrm{C}$ & $\mathrm{C}$ & $\mathrm{T}$ & $\mathrm{C}$ & $\mathrm{T}$ & $\mathrm{A}$ & $\mathrm{T}$ & $\mathrm{T}$ & $\mathrm{C}$ \\
\hline Ps. dawaina & MM14 F0015 & $\mathrm{T}$ & $\mathrm{C}$ & $\mathrm{A}$ & $\mathrm{T}$ & $\mathrm{T}$ & $\mathrm{C}$ & $\mathrm{C}$ & $\mathrm{T}$ & $\mathrm{C}$ & $\mathrm{T}$ & A & $\mathrm{C}$ & $\mathrm{T}$ & $\mathrm{T}$ \\
\hline Ps. jiangxiensis & LC4479 & $\mathrm{T}$ & $\mathrm{T}$ & $\mathrm{G}$ & $\mathrm{G}$ & $\mathrm{T}$ & $\mathrm{C}$ & $\mathrm{T}$ & $\mathrm{C}$ & $\mathrm{T}$ & $\mathrm{C}$ & $\mathrm{A}$ & $\mathrm{C}$ & $\mathrm{T}$ & $\mathrm{T}$ \\
\hline Ps. kawthaungina & MM14 F0083 & $\mathrm{T}$ & $\mathrm{C}$ & $\mathrm{A}$ & $\mathrm{T}$ & $\mathrm{T}$ & $\mathrm{C}$ & $\mathrm{C}$ & $\mathrm{T}$ & $\mathrm{C}$ & $\mathrm{T}$ & $\mathrm{A}$ & $\mathrm{C}$ & $\mathrm{T}$ & $\mathrm{T}$ \\
\hline Ps. myanmarina & NBRC 112264 & $\mathrm{~T}$ & $\mathrm{~T}$ & $\mathrm{G}$ & $\mathrm{G}$ & $\mathrm{T}$ & $\mathrm{C}$ & $\mathrm{T}$ & $\mathrm{C}$ & $\mathrm{T}$ & $\mathrm{C}$ & A & $\mathrm{C}$ & $\mathrm{T}$ & $\mathrm{T}$ \\
\hline Ps. rhizophorae & MFLUCC 17-560 & $\mathrm{T}$ & $\mathrm{C}$ & $\mathrm{A}$ & $\mathrm{T}$ & $\mathrm{C}$ & $\mathrm{G}$ & $\mathrm{C}$ & $\mathrm{T}$ & $\mathrm{C}$ & $\mathrm{T}$ & $\mathrm{C}$ & $\mathrm{C}$ & $\mathrm{T}$ & $\mathrm{T}$ \\
\hline Ps. simitheae & MFLUCC 12-0121 & $\mathrm{C}$ & $\mathrm{C}$ & $\mathrm{A}$ & $\mathrm{T}$ & $\mathrm{T}$ & $\mathrm{C}$ & $\mathrm{C}$ & $\mathrm{T}$ & $\mathrm{C}$ & $\mathrm{T}$ & $\mathrm{G}$ & $\mathrm{C}$ & $\mathrm{A}$ & $\mathrm{T}$ \\
\hline Ps. taiwanensis & NTUCC 17-002.1 & $\mathrm{C}$ & $\mathrm{C}$ & $\mathrm{A}$ & $\mathrm{T}$ & $\mathrm{T}$ & $\mathrm{C}$ & $\mathrm{C}$ & $\mathrm{T}$ & $\mathrm{C}$ & $\mathrm{T}$ & A & $\mathrm{C}$ & $\mathrm{T}$ & $\mathrm{T}$ \\
\hline Ps. thailandica & MFLUCC 17-1724 & $\mathrm{T}$ & $\mathrm{C}$ & $\mathrm{A}$ & $\mathrm{T}$ & $\mathrm{C}$ & $\mathrm{C}$ & $\mathrm{C}$ & $\mathrm{T}$ & $\mathrm{C}$ & $\mathrm{T}$ & $\mathrm{C}$ & $\mathrm{C}$ & $\mathrm{T}$ & $\mathrm{T}$ \\
\hline \multirow{2}{*}{ Taxon } & \multirow{2}{*}{ Strain } & \multicolumn{14}{|c|}{ EF1a } \\
\hline & & 99 & 100 & 102 & 105 & 114 & 115 & 123 & 125 & 127 & 128 & 134 & 137 & 140 & 150 \\
\hline Pseudopestalotiopsis avucenniae & MFLUCC 17-0434 & $\mathrm{T}$ & $\mathrm{C}$ & $\mathrm{A}$ & $\mathrm{G}$ & - & - & $\mathrm{A}$ & $\mathrm{C}$ & $\mathrm{T}$ & $\mathrm{C}$ & $\mathrm{C}$ & $\mathrm{G}$ & $\mathrm{C}$ & $\mathrm{C}$ \\
\hline Ps. curvatispora & MFLUCC 17-1722 & $\mathrm{T}$ & $\mathrm{G}$ & $\mathrm{T}$ & $\mathrm{A}$ & $\mathrm{A}$ & $\mathrm{A}$ & A & $\mathrm{T}$ & $\mathrm{C}$ & $\mathrm{C}$ & $\mathrm{A}$ & $\mathrm{A}$ & $\mathrm{T}$ & $\mathrm{C}$ \\
\hline Ps. dawaina & MM14 F0015 & $\mathrm{C}$ & - & - & $\mathrm{A}$ & - & - & $\mathrm{T}$ & $\mathrm{C}$ & $\mathrm{C}$ & $\mathrm{C}$ & $\mathrm{C}$ & $\mathrm{A}$ & $\mathrm{T}$ & $\mathrm{A}$ \\
\hline Ps. jiangxiensis & LC4479 & $\mathrm{T}$ & $\mathrm{C}$ & $\mathrm{A}$ & $\mathrm{G}$ & - & - & $\mathrm{A}$ & $\mathrm{C}$ & $\mathrm{T}$ & $\mathrm{C}$ & $\mathrm{C}$ & $\mathrm{G}$ & $\mathrm{C}$ & $\mathrm{C}$ \\
\hline Ps. kawthaungina & MM14 F0083 & $\mathrm{T}$ & $\mathrm{C}$ & $\mathrm{A}$ & $\mathrm{G}$ & - & - & A & $\mathrm{C}$ & $\mathrm{T}$ & $\mathrm{C}$ & $\mathrm{C}$ & $\mathrm{G}$ & $\mathrm{C}$ & $\mathrm{C}$ \\
\hline Ps. myanmarina & NBRC 112264 & $\mathrm{~T}$ & $\mathrm{C}$ & $\mathrm{A}$ & $\mathrm{G}$ & - & - & $\mathrm{A}$ & $\mathrm{C}$ & $\mathrm{T}$ & $\mathrm{C}$ & $\mathrm{C}$ & $\mathrm{G}$ & $\mathrm{C}$ & $\mathrm{C}$ \\
\hline Ps. rhizophorae & MFLUCC 17-560 & $\mathrm{T}$ & $\mathrm{C}$ & $\mathrm{T}$ & A & - & - & $\mathrm{G}$ & $\mathrm{C}$ & $\mathrm{C}$ & $\mathrm{C}$ & $\mathrm{C}$ & $\mathrm{A}$ & $\mathrm{T}$ & $\mathrm{C}$ \\
\hline Ps. simitheae & MFLUCC 12-0121 & $\mathrm{T}$ & $\mathrm{C}$ & $\mathrm{T}$ & $\mathrm{A}$ & - & - & $\mathrm{G}$ & $\mathrm{C}$ & $\mathrm{C}$ & $\mathrm{C}$ & $\mathrm{C}$ & $\mathrm{A}$ & $\mathrm{T}$ & $\mathrm{C}$ \\
\hline Ps. taiwanensis & NTUCC 17-002.1 & $\mathrm{T}$ & $\mathrm{C}$ & $\mathrm{T}$ & $\mathrm{A}$ & $\mathrm{A}$ & - & $\mathrm{A}$ & $\mathrm{C}$ & $\mathrm{T}$ & $\mathrm{T}$ & $\mathrm{C}$ & $\mathrm{G}$ & $\mathrm{T}$ & $\mathrm{C}$ \\
\hline Ps. thailandica & MFLUCC 17-1724 & $\mathrm{T}$ & $\mathrm{C}$ & $\mathrm{T}$ & A & - & - & $\mathrm{G}$ & $\mathrm{C}$ & $\mathrm{C}$ & $\mathrm{C}$ & $\mathrm{C}$ & $\mathrm{A}$ & $\mathrm{T}$ & $\mathrm{C}$ \\
\hline \multirow{2}{*}{ Taxon } & \multirow{2}{*}{ Strain } & \multicolumn{14}{|c|}{ EF1a } \\
\hline & & 151 & 152 & 157 & 167 & 172 & 176 & 186 & 252 & 267 & 268 & 269 & 270 & 271 & 272 \\
\hline Pseudopestalotiopsis avucenniae & MFLUCC 17-0434 & $\mathrm{C}$ & A & $\mathrm{A}$ & $\mathrm{C}$ & $\mathrm{A}$ & $\mathrm{G}$ & $\mathrm{T}$ & A & $\mathrm{C}$ & $\mathrm{A}$ & $\mathrm{G}$ & $\mathrm{C}$ & $\mathrm{A}$ & $\mathrm{A}$ \\
\hline Ps. curvatispora & MFLUCC 17-1722 & - & - & $\mathrm{C}$ & $\mathrm{T}$ & $\mathrm{A}$ & $\mathrm{C}$ & $\mathrm{C}$ & A & $\mathrm{C}$ & $\mathrm{A}$ & $\mathrm{G}$ & $\mathrm{C}$ & $\mathrm{A}$ & $\mathrm{A}$ \\
\hline Ps. dawaina & MM14 F0015 & $\mathrm{G}$ & A & $\mathrm{A}$ & $\mathrm{T}$ & $\mathrm{A}$ & $\mathrm{C}$ & $\mathrm{C}$ & $\mathrm{A}$ & $\mathrm{C}$ & $\mathrm{A}$ & $\mathrm{G}$ & $\mathrm{C}$ & A & $\mathrm{A}$ \\
\hline Ps. jiangxiensis & LC4479 & $\mathrm{C}$ & $\mathrm{A}$ & $\mathrm{A}$ & $\mathrm{T}$ & $\mathrm{A}$ & $\mathrm{C}$ & $\mathrm{T}$ & $\mathrm{T}$ & $\mathrm{C}$ & $\mathrm{A}$ & $\mathrm{G}$ & $\mathrm{C}$ & $\mathrm{A}$ & $\mathrm{A}$ \\
\hline Ps. kawthaungina & MM14 F0083 & $\mathrm{C}$ & $\mathrm{N}$ & $\mathrm{A}$ & $\mathrm{T}$ & $\mathrm{A}$ & $\mathrm{C}$ & $\mathrm{T}$ & $\mathrm{A}$ & $\mathrm{C}$ & $\mathrm{A}$ & $\mathrm{G}$ & $\mathrm{C}$ & $\mathrm{A}$ & $\mathrm{A}$ \\
\hline Ps. myanmarina & NBRC 112264 & $\mathrm{C}$ & A & $\mathrm{A}$ & $\mathrm{T}$ & $\mathrm{A}$ & $\mathrm{C}$ & $\mathrm{T}$ & $\mathrm{A}$ & $\mathrm{C}$ & $\mathrm{A}$ & $\mathrm{G}$ & $\mathrm{C}$ & $\mathrm{A}$ & $\mathrm{A}$ \\
\hline Ps. rhizophorae & MFLUCC 17-560 & $\mathrm{G}$ & $\mathrm{A}$ & $\mathrm{A}$ & $\mathrm{T}$ & $\mathrm{C}$ & $\mathrm{C}$ & $\mathrm{C}$ & $\mathrm{A}$ & - & - & - & - & - & - \\
\hline Ps. simitheae & MFLUCC 12-0121 & $\mathrm{G}$ & $\mathrm{A}$ & $\mathrm{A}$ & $\mathrm{T}$ & $\mathrm{C}$ & $\mathrm{C}$ & $\mathrm{C}$ & $\mathrm{A}$ & - & - & - & - & - & - \\
\hline Ps. taiwanensis & NTUCC 17-002.1 & $\mathrm{A}$ & $\mathrm{A}$ & $\mathrm{A}$ & $\mathrm{T}$ & $\mathrm{A}$ & $\mathrm{C}$ & $\mathrm{T}$ & $\mathrm{A}$ & $\mathrm{C}$ & $\mathrm{A}$ & $\mathrm{G}$ & $\mathrm{C}$ & $\mathrm{A}$ & $\mathrm{A}$ \\
\hline Ps. thailandica & MFLUCC 17-1724 & $\mathrm{G}$ & A & $\mathrm{A}$ & $\mathrm{T}$ & $\mathrm{C}$ & $\mathrm{C}$ & $\mathrm{C}$ & $\mathrm{A}$ & - & - & - & - & - & - \\
\hline
\end{tabular}


Supplementary Table 3 Continued.

\begin{tabular}{|c|c|c|c|c|c|c|c|c|c|c|c|c|c|c|c|}
\hline \multirow{2}{*}{ Taxon } & \multirow{2}{*}{ Strain } & \multicolumn{14}{|c|}{ EF1a } \\
\hline & & 273 & 274 & 275 & 276 & 277 & 278 & 279 & 280 & 281 & 282 & 283 & 286 & 290 & 304 \\
\hline Pseudopestalotiopsis avucenniae & MFLUCC 17-0434 & $\mathrm{C}$ & - & - & - & $\mathrm{C}$ & $\mathrm{A}$ & $\mathrm{T}$ & $\mathrm{G}$ & $\mathrm{C}$ & $\mathrm{A}$ & $\mathrm{C}$ & $\mathrm{C}$ & $\mathrm{T}$ & $\mathrm{A}$ \\
\hline Ps. curvatispora & MFLUCC 17-1722 & $\mathrm{C}$ & - & - & - & $\mathrm{C}$ & A & $\mathrm{T}$ & $\mathrm{G}$ & $\mathrm{C}$ & A & $\mathrm{C}$ & $\mathrm{T}$ & $\mathrm{T}$ & A \\
\hline Ps. dawaina & MM14 F0015 & $\mathrm{C}$ & $\mathrm{T}$ & $\mathrm{A}$ & $\mathrm{C}$ & $\mathrm{C}$ & $\mathrm{A}$ & $\mathrm{T}$ & $\mathrm{G}$ & $\mathrm{C}$ & A & $\mathrm{C}$ & $\mathrm{C}$ & $\mathrm{T}$ & $\mathrm{A}$ \\
\hline Ps. jiangxiensis & LC4479 & $\mathrm{C}$ & - & - & - & $\mathrm{C}$ & $\mathrm{A}$ & $\mathrm{T}$ & $\mathrm{G}$ & $\mathrm{C}$ & $\mathrm{A}$ & $\mathrm{C}$ & $\mathrm{C}$ & $\mathrm{T}$ & $\mathrm{A}$ \\
\hline Ps. kawthaungina & MM14 F0083 & $\mathrm{C}$ & - & - & - & $\mathrm{C}$ & $\mathrm{A}$ & $\mathrm{T}$ & $\mathrm{G}$ & $\mathrm{C}$ & $\mathrm{A}$ & $\mathrm{C}$ & $\mathrm{C}$ & $\mathrm{T}$ & $\mathrm{A}$ \\
\hline Ps. myanmarina & NBRC 112264 & $\mathrm{C}$ & - & - & - & $\mathrm{C}$ & $\mathrm{A}$ & $\mathrm{T}$ & $\mathrm{G}$ & $\mathrm{C}$ & $\mathrm{A}$ & $\mathrm{C}$ & $\mathrm{C}$ & $\mathrm{T}$ & $\mathrm{A}$ \\
\hline Ps. rhizophorae & MFLUCC 17-560 & - & - & - & - & - & - & - & - & - & - & - & $\mathrm{C}$ & $\mathrm{C}$ & $\mathrm{A}$ \\
\hline Ps. simitheae & MFLUCC 12-0121 & - & - & - & - & - & - & - & - & - & - & - & $\mathrm{C}$ & $\mathrm{T}$ & $\mathrm{C}$ \\
\hline Ps. taiwanensis & NTUCC 17-002.1 & $\mathrm{C}$ & - & - & - & $\mathrm{C}$ & $\mathrm{A}$ & $\mathrm{T}$ & $\mathrm{G}$ & $\mathrm{C}$ & A & $\mathrm{C}$ & $\mathrm{C}$ & $\mathrm{T}$ & A \\
\hline Ps. thailandica & MFLUCC 17-1724 & - & - & - & - & - & - & - & - & - & - & - & $\mathrm{C}$ & $\mathrm{C}$ & $\mathrm{A}$ \\
\hline \multirow{2}{*}{ Taxon } & \multirow{2}{*}{ Strain } & \multicolumn{14}{|c|}{ 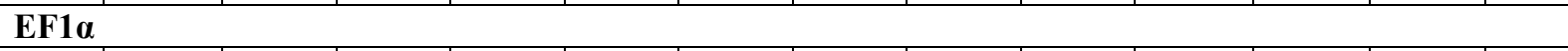 } \\
\hline & & 306 & 308 & 312 & 318 & 321 & 343 & 475 & 476 & 478 & 479 & 480 & 484 & 487 & 488 \\
\hline Pseudopestalotiopsis avucenniae & MFLUCC 17-0434 & $\mathrm{T}$ & $\mathrm{A}$ & $\mathrm{C}$ & $\mathrm{C}$ & $\mathrm{C}$ & $\mathrm{C}$ & $\mathrm{G}$ & $\mathrm{T}$ & $\mathrm{C}$ & $\mathrm{C}$ & $\mathrm{T}$ & $\mathrm{C}$ & $\mathrm{G}$ & $\mathrm{C}$ \\
\hline Ps. curvatispora & MFLUCC 17-1722 & $\mathrm{C}$ & $\mathrm{A}$ & $\mathrm{C}$ & $\mathrm{C}$ & $\mathrm{C}$ & $\mathrm{C}$ & $\mathrm{T}$ & $\mathrm{T}$ & $\mathrm{C}$ & $\mathrm{C}$ & $\mathrm{T}$ & A & A & $\mathrm{C}$ \\
\hline Ps. dawaina & MM14 F0015 & $\mathrm{C}$ & $\mathrm{A}$ & $\mathrm{C}$ & $\mathrm{C}$ & $\mathrm{C}$ & $\mathrm{C}$ & $\mathrm{T}$ & $\mathrm{T}$ & $\mathrm{C}$ & $\mathrm{T}$ & $\mathrm{T}$ & $\mathrm{C}$ & $\mathrm{A}$ & $\mathrm{C}$ \\
\hline Ps. jiangxiensis & LC4479 & $\mathrm{T}$ & $\mathrm{A}$ & $\mathrm{C}$ & $\mathrm{C}$ & $\mathrm{C}$ & $\mathrm{C}$ & $\mathrm{G}$ & $\mathrm{T}$ & $\mathrm{C}$ & $\mathrm{C}$ & $\mathrm{T}$ & $\mathrm{C}$ & $\mathrm{G}$ & $\mathrm{C}$ \\
\hline Ps. kawthaungina & MM14 F0083 & $\mathrm{C}$ & $\mathrm{A}$ & $\mathrm{C}$ & $\mathrm{C}$ & $\mathrm{C}$ & $\mathrm{C}$ & $\mathrm{T}$ & $\mathrm{T}$ & $\mathrm{A}$ & $\mathrm{C}$ & $\mathrm{T}$ & $\mathrm{C}$ & $\mathrm{G}$ & $\mathrm{T}$ \\
\hline Ps. myanmarina & NBRC 112264 & $\mathrm{~T}$ & $\mathrm{~A}$ & $\mathrm{C}$ & $\mathrm{C}$ & $\mathrm{C}$ & $\mathrm{C}$ & $\mathrm{G}$ & $\mathrm{T}$ & $\mathrm{C}$ & $\mathrm{C}$ & $\mathrm{T}$ & $\mathrm{C}$ & $\mathrm{G}$ & $\mathrm{C}$ \\
\hline Ps. rhizophorae & MFLUCC 17-560 & $\mathrm{C}$ & $\mathrm{G}$ & $\mathrm{C}$ & $\mathrm{T}$ & A & $\mathrm{T}$ & $\mathrm{T}$ & $\mathrm{C}$ & $\mathrm{C}$ & $\mathrm{T}$ & $\mathrm{C}$ & $\mathrm{C}$ & $\mathrm{G}$ & $\mathrm{C}$ \\
\hline Ps. simitheae & MFLUCC 12-0121 & $\mathrm{C}$ & $\mathrm{A}$ & $\mathrm{C}$ & $\mathrm{C}$ & $\mathrm{C}$ & $\mathrm{C}$ & $\mathrm{T}$ & $\mathrm{C}$ & $\mathrm{C}$ & $\mathrm{T}$ & $\mathrm{T}$ & $\mathrm{C}$ & $\mathrm{G}$ & $\mathrm{C}$ \\
\hline Ps. taiwanensis & NTUCC 17-002.1 & $\mathrm{C}$ & $\mathrm{A}$ & $\mathrm{T}$ & $\mathrm{C}$ & $\mathrm{C}$ & $\mathrm{C}$ & $\mathrm{T}$ & $\mathrm{T}$ & $\mathrm{C}$ & $\mathrm{C}$ & $\mathrm{C}$ & $\mathrm{C}$ & $\mathrm{A}$ & $\mathrm{C}$ \\
\hline Ps. thailandica & MFLUCC 17-1724 & $\mathrm{C}$ & $\mathrm{G}$ & $\mathrm{C}$ & $\mathrm{T}$ & A & $\mathrm{T}$ & $\mathrm{T}$ & $\mathrm{C}$ & $\mathrm{C}$ & $\mathrm{T}$ & $\mathrm{C}$ & $\mathrm{C}$ & $\mathrm{G}$ & $\mathrm{C}$ \\
\hline \multirow{2}{*}{ Taxon } & \multirow{2}{*}{ Strain } & \multicolumn{14}{|c|}{ EF1a } \\
\hline & & 491 & 492 & 493 & 496 & 500 & 501 & 504 & 510 & 512 & 521 & 524 & & & \\
\hline Pseudopestalotiopsis avucenniae & MFLUCC 17-0434 & A & $\mathrm{G}$ & $\mathrm{C}$ & $\mathrm{C}$ & $\mathrm{G}$ & $\mathrm{A}$ & $\mathrm{T}$ & $\mathrm{T}$ & - & $\mathrm{T}$ & $\mathrm{C}$ & & & \\
\hline Ps. curvatispora & MFLUCC 17-1722 & $\mathrm{A}$ & $\mathrm{G}$ & $\mathrm{C}$ & $\mathrm{C}$ & $\mathrm{G}$ & $\mathrm{C}$ & $\mathrm{T}$ & $\mathrm{C}$ & - & $\mathrm{T}$ & $\mathrm{T}$ & & & \\
\hline Ps. dawaina & MM14 F0015 & A & $\mathrm{G}$ & $\mathrm{T}$ & $\mathrm{C}$ & $\mathrm{G}$ & $\mathrm{A}$ & $\mathrm{T}$ & $\mathrm{T}$ & $\mathrm{T}$ & $\mathrm{T}$ & $\mathrm{T}$ & & & \\
\hline Ps. jiangxiensis & LC4479 & $\mathrm{A}$ & $\mathrm{G}$ & $\mathrm{C}$ & $\mathrm{C}$ & $\mathrm{G}$ & $\mathrm{A}$ & $\mathrm{T}$ & $\mathrm{T}$ & - & $\mathrm{T}$ & $\mathrm{C}$ & & & \\
\hline Ps. kawthaungina & MM14 F0083 & A & $\mathrm{A}$ & $\mathrm{C}$ & $\mathrm{C}$ & $\mathrm{G}$ & $\mathrm{A}$ & $\mathrm{G}$ & $\mathrm{T}$ & - & $\mathrm{T}$ & $\mathrm{C}$ & & & \\
\hline Ps. myanmarina & NBRC 112264 & $\mathrm{~A}$ & $\mathrm{G}$ & $\mathrm{C}$ & $\mathrm{C}$ & $\mathrm{G}$ & $\mathrm{A}$ & $\mathrm{T}$ & $\mathrm{T}$ & - & $\mathrm{T}$ & $\mathrm{C}$ & & & \\
\hline Ps. rhizophorae & MFLUCC 17-560 & $\mathrm{T}$ & $\mathrm{C}$ & $\mathrm{C}$ & $\mathrm{C}$ & $\mathrm{G}$ & $\mathrm{A}$ & $\mathrm{T}$ & $\mathrm{T}$ & - & $\mathrm{C}$ & $\mathrm{C}$ & & & \\
\hline Ps. simitheae & MFLUCC 12-0121 & A & $\mathrm{C}$ & $\mathrm{G}$ & $\mathrm{C}$ & $\mathrm{A}$ & $\mathrm{A}$ & $\mathrm{T}$ & $\mathrm{T}$ & - & $\mathrm{C}$ & $\mathrm{C}$ & & & \\
\hline Ps. taiwanensis & NTUCC 17-002.1 & $\mathrm{A}$ & $\mathrm{G}$ & $\mathrm{C}$ & $\mathrm{T}$ & $\mathrm{G}$ & $\mathrm{A}$ & $\mathrm{T}$ & $\mathrm{T}$ & - & $\mathrm{T}$ & $\mathrm{G}$ & & & \\
\hline Ps. thailandica & MFLUCC 17-1724 & $\mathrm{T}$ & $\mathrm{C}$ & $\mathrm{C}$ & $\mathrm{C}$ & $\mathrm{G}$ & $\mathrm{A}$ & $\mathrm{T}$ & $\mathrm{T}$ & - & $\mathrm{C}$ & $\mathrm{C}$ & & & \\
\hline
\end{tabular}

